\title{
CORAL REEFS OF THE SULTANATE OF OMAN
}

\author{
BY
}

\author{
RODNEY V. SALM
}

\section{INTRODUCTION}

\section{Background}

Corals and coral communities in Oman have been described for the Musandam area in the north (Glynn 1983), the Muscat Area (Green 1983, 1984; Green \& Keech 1986; Glynn, 1983; Salm \& Sheppard 1986), Masirah Island (Glynn 1983), Dhofar in the south (Barrat 1984, Glynn 1983), and for Oman generally (Sheppard \& Salm 1988).

Coral diversity has been mapped for the Muscat Area (IUCN 1986) and, in more detail, for specific sites within it (Salm 1986a), for the southern Gulf of Oman (IUCN 1988), Dhofar (IUCN 1989b), and Musandam (IUCN 1991). Coral records of the IUCN Coastal Zone Management (CZM) Project were incorporated into a computerized database using DBase III Plus for 185 sites spanning the entire coast of the Sultanate (Appendix 1). The database also classifies these sites by the type of coral assemblage (i.e., coral reef with framework development or rock with non-reef coral community), and provides general descriptions of the site and information on the uses and threats to corals there.

This report is an interpretation of the database, and a second general environmental classification database that provides details of uses and management issues for 330 sites, of which 246 have coral communities (Appendix 2). It presents additional information on the corals of Oman, resulting from studies directed specifically toward the formulation of coastal zone management plans (CZMPs) and overview reports covering the entire coast of the Sultanate.

IUCN Coastal Zone Management Project

P.O. Box 550, Muscat, Sultanate of Oman

Present address:

Coordinator, Marine \& Coastal Programme

IUCN Eastern Africa Regional Africa

P.O. Box 68200, Nairobi, Kenya 


\section{Objectives}

Coral communities were surveyed between November 1984 and June 1992. The aim of these surveys was to identify areas that were most valuable for conservation, and hence least suitable for major coastal works, such as marinas, fishing boat harbours, or effluent outfalls from desalination plants, sewage works or other sources.

The objectives of the surveys were to:

1) compile a comprehensive list of corals found at each study site;

2) map the distribution of coral communities;

3) select candidate sites for conservation of coral communities based on coral diversity (measured as richness of coral genera and species), percentage cover of corals, degree of reef framework development, presence of unusual corals or coral formations, and/or representativeness of coral assemblages for each section of coast;

4) identify uses of coral environments and their impacts, and other natural or peoplerelated threats;

5) define management issues, and specify actions and responsibilities to resolve these; and

6) compile named collections of corals for presentation to the Oman Natural History Museum.

\section{Methods}

The study area includes the entire coast of Oman, extending from the Arabian Gulf coast in the north to the Yemen border in the south, and including all offshore islands.

The likely location of coral communities was determined from aerial photographs, helicopter overflights of most of the study area, and boat and underwater reconnaissance.

Corals were identified and recorded in situ, while snorkeling or scuba diving, at a total of 170 specific sites (Figure 1). Regional coral records were supplemented by beach specimens or underwater sightings made in addition to the detailed listings. Representative collections of corals from each region (Musandam, Muscat area, Masirah area, Dhofar) were made for reference. Corals that could not be identified underwater were collected, cleaned, and identified by comparison with the reference collections, or from the following texts: Burchand (1980), Scheer \& Pillai (1983), Veron \& Pichon (1976, 1980, 1982), Veron \& Wallace (1984), Veron, Pichon \& Wijsman-Best (1977), Wells (1956). All coral specimens were presented to the Oman Natural History Museum (contact: Curator, Oman Natural History Museum, Ministry of National Heritage and Culture, P.O. Box 668, Muscat, Sultanate of Oman).

Corals were recorded by the following standard procedure developed to enable rapid comparative surveys of numerous reef areas. 
Upon entry into the sea, several minutes were devoted to orientation and recording the nature of substrate and coral community (percent cover, angle of slope, presence of reef framework, dominant and predominant corals). Next, corals were recorded continuously in sequential five minute intervals onto a plastic slate for generally 30 minutes. This procedure yielded a characteristic genus-discovery curve (total genera $\mathrm{x}$ five minute interval) for each study site (Figures 2, 3, 4).

This genus-discovery method, described in more detail below, was developed by me to enable rapid survey of reefs and facilitate comparative assessment of coral diversity over vast tracts of the Indonesian Archipelago. The principle is one of non-random focussed search for different coral genera over a 30 minute period. The search covers as many different zones and habitats as possible (typically in this order: reef crest, slope, edge, gullies, walls, overhangs, caves, reef-flat) to yield the greatest possible variety of corals during the time period. Corals are identified to genus in situ, and recorded onto a plastic slate. Each 5-minute interval is marked. This yields a total genus count per 30 minutes for each coral community (the richness index), and a count of additional genera per 5minute interval. The yield of additional genera drops with each successive 5-minute interval.

The total number of genera plotted over the six successive 5-minute interval numbers yields a characteristic genus-discovery (G-D) curve that reflects the influence of different habitats on patterns of genus discovery.

For example, coral communities in disturbed environments (rock fall areas, reef crests with periodic heavy wave damage) support numerous small coral colonies in mixed assemblages. As the recorder does not need to move far to discover additional genera, there is a rapid initial yield of different corals. The result is a typical exponential G-D curve.

In sheltered environments where coral suffers little disturbance, individual colonies of Porites and Acropora can grow to extremely large sizes. Acropora, in particular, can monopolize vast areas. Here, the search time for different genera is prolonged, yielding a characteristic flat G-D curve. However, if the substrate shows great morphological variation (e.g., spurs, ridges, deep gullies, walls), coral distribution will be patchy. The resulting G-D curve will be noticeably stepped (i.e., there is a rapid initial yield of additional genera in each new habitat followed by a levelling off until the adjacent habitat is reached). The inflection points in this stepped type G-D curve correspond to the points of change from one habitat to another.

The coral assemblages around the Krakatau Islands in Indonesia reflect these three characteristic G-D curves well (Salm, unpublished data; Salm, Sukotjo, Genolagani 1982). 


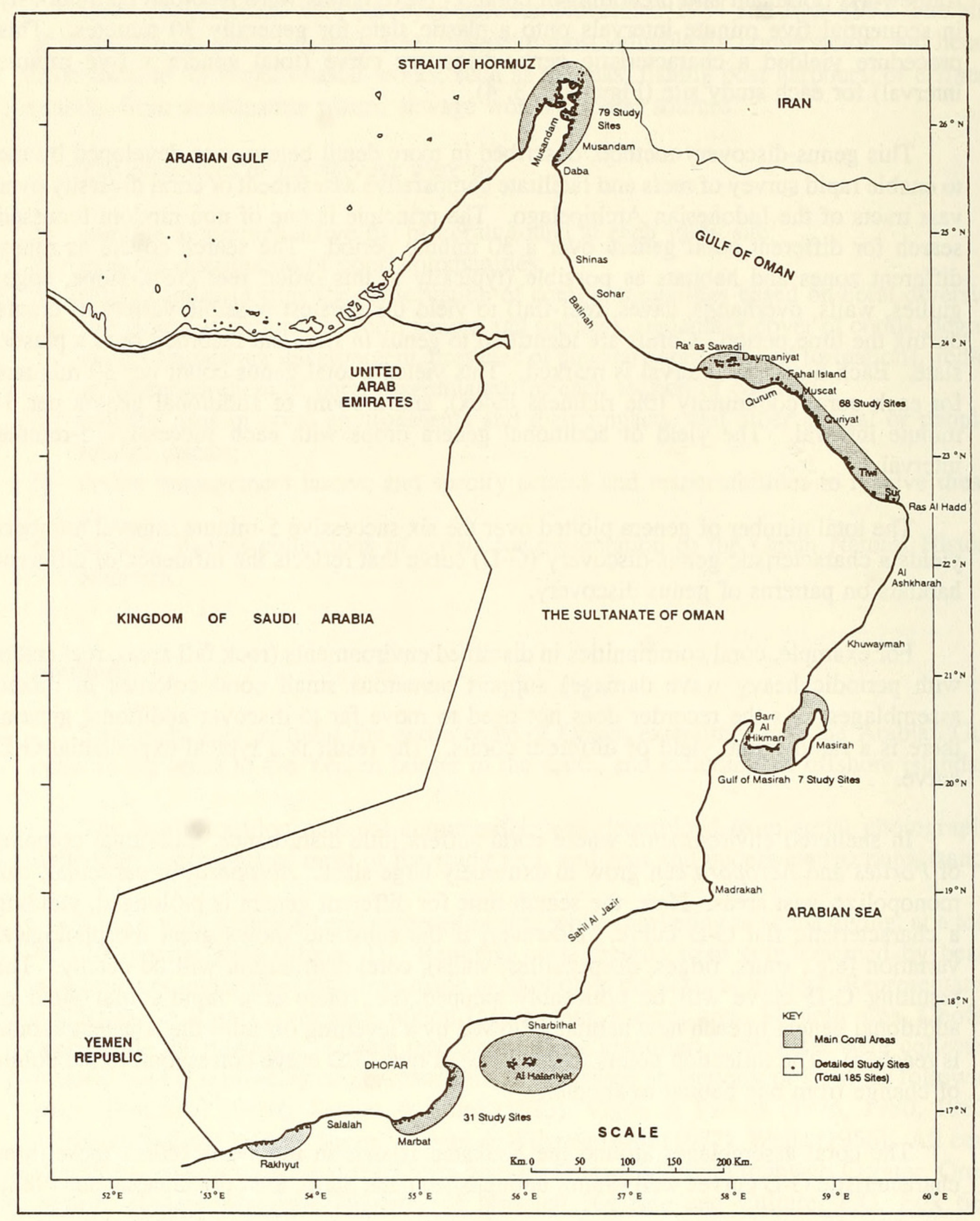

Figure 1. Distribution of coral and coral study sites in Oman 


\section{DISTRIBUTION AND NATURE OF CORAL COMMUNITIES}

\section{Influences on Coral Occurrence and Growth}

Sheppard and Salm (1988) state that only three areas on the mainland support significant coral growth: Musandam, Muscat area and Dhofar. However, it is clear from the coastal zone management studies that major coral growth occurs in four regions in Oman: the Musandam Peninsula and adjacent rocky coast in the north, where coral growth is most prolific; the rocky shores, bays and islands adjacent to the Muscat area, where overall coral diversity is comparable to Musandam but greater at individual sites; the strait west of Masirah Island and adjacent northern Gulf of Masirah, which has the largest areas of continuous coral growth but low diversity; and a number of sheltered bays along mainland Dhofar in the south and the Al Halaniyat Islands offshore, where relict populations of some corals occur that are found nowhere else in the Sultanate. Other parts of the Oman coast either lack corals or support limited growth of small scattered colonies. In some instances the absence of suitable stable substrate precludes the settlement and growth of coral, although seasonal upwelling of cold water, vigorous algal growth, and heavy wave action must also contribute, such as along most of the Arabian Sea coast.

It is interesting that corals had a wider distribution along the Oman coast during Pleistocene times than at present. Beach gravel deposits of this period found from Ra's al Hadd west to beyond Sur contain corals no longer found alive here, but found in Dhofar to the south (Euphyllia, Tubipora), and a variety of molluscs (Tridacna maxima, Lambis lambis, Cypraecassis rufa) that are common associates of coral communities elsewhere, but which are no longer found living anywhere in Oman.

There are differences between the corals and coral fishes in the Gulf of Oman and those in the Arabian Sea that appear to be linked to the different oceanographic regimes influencing the coasts north and south of Ra's al Hadd, the eastern point of Oman and the break between the two water masses. During summer, the coast south of Ra's al Hadd bears the brunt of the southwest monsoon winds, swell and upwelling cold water. At this time, sea temperatures reach their highest levels north of Ra's al Hadd.

Complex winds and currents occasionally cause the upwelling of cold water in the Muscat area. This is particularly noticeable in summer when seawater temperatures can drop by as much as $10^{\circ} \mathrm{C}$ in a day or two. Both summer and winter upwellings are very localized in the Muscat area, and can cause red tides.

The Dhofar upwellings are quite different. These are fairly constant upwellings that continue for 4-5 months during the summer monsoon. This is a period of tremendously rough, cold, green seas when seaweed growth proliferates on all rocky coasts of the southern region. These seaweeds grow fast when upwellings flood the seas with nutrientrich cool water $\left(16-19^{\circ} \mathrm{C}\right)$, some reaching $1.5-2 \mathrm{~m}$ in just a few months. 
Large patches of dead coral are a common sight and source of speculation in the Muscat area and Musandam. Acanthaster planci outbreaks, and temperature-induced bleaching, have killed some coral patches. Entangling fishing nets have destroyed others, and silt-laden flood waters almost certainly contribute to coral death. It is also likely that the summer upwellings contribute to localized coral mortality by sudden large drops in temperature, stressing the corals beyond their limits of tolerance.

\section{The Nature of Corals and Coral Communities}

Coral genera found by the CZM Project and other sources are listed in Table 1 for each of the four main coral regions of the Sultanate (Figure 1).

Hard-base corals (those requiring a hard substrate on which to settle and grow) are confined largely to islands, rocky shoals and reefs, and the rocky coasts of Musandam, the Muscat area, the strait west of Masirah Island and northern Gulf of Masirah, and sheltered sections of the Dhofar coast, including the Al Halaniyat Islands (IUCN 1986, 1988, 1989a,b, 1991; Salm 1989; Sheppard \& Salm, 1988).

Coral communities tend to be confined to above a maximum depth of $12-18 \mathrm{~m}$, but the percentage cover of live corals drops abruptly below $10 \mathrm{~m}$ from $75 \%$ or more to less than $40 \%$, and seldom reaches $10 \%$ at a depth of $15 \mathrm{~m}$. Certain generally ahermatypic (non reef-building) corals will grow on rocky outcrops down to or deeper than $30 \mathrm{~m}$ (Culicia, Balanophyllia, Polycyathus, Paracyathus, Phyllangia, Rhizopsammia, Leptoseris). The maximum depth of corals is determined by at least three parameters: the general progression from rock to soft substrate below $12-20 \mathrm{~m}$, the usual presence of a thermocline at $10-15 \mathrm{~m}$ (occasionally less) below which the water is both turbid and cold, and the generally murky water. Underwater visibility is unpredictable in the Sultanate, changing daily, and generally less than $10-12 \mathrm{~m}$.

Most corals grow directly on bedrock or on the dead parts of other corals to form a shallow veneer over the underlying substrate. However, conditions favour the build-up of small framework reefs in places. These rudimentary reefs are restricted to sheltered coasts in bays and coves, and along the leeward sides of headlands and islands.

Porites is the dominant builder of framework reefs throughout the Sultanate. Adjacent colonies fuse in the shallows to form flat-topped platforms (reef-flats) that may be settled by other coral species. These fused colonies attain a size of two to four metres in diameter (maximum five metres) along the seaward border of the reef-flat. The large Porites colonies and Porites dominated reefs probably form the oldest continuously living reefs in the Sultanate.

Pocillopora damicornis commonly forms nearly monospecific banks of living coral to between two and three metres thick. All living corals on some of these rudimentary $P$. damicornis reefs have been extensively or totally killed, and the reefs are being 
recolonized by algae, alcyonarians, Acropora, Pocillopora and other corals.

Montipora foliosa forms large reefs in the northern Gulf of Masirah. Acropora and Hydnophora may occasionally also form small rudimentary reef patches with a framework 1-2 m thick. In Musandam, Pavona cactus and Montipora sp. commonly form patches of reef framework more than one metre thick. None of these corals is noted as building framework reefs by Sheppard and Salm (1988).

As noted by Glynn (1983), acroporid reefs were once extensive and widely distributed. Those off the Daymaniyat and Fahal Islands, in Bandar Jissah and at many other sites along the Musandam and Muscat coasts were totally killed, presumably by $A$. planci, but are being recolonized by $P$. damicornis, Acropora and other corals.

Coral communities conform to six main types, each of which has a characteristic genus-discovery curve (see p. 3 for explanation).

1) Rudimentary patch or fringing reefs dominated by Porites with few small scattered colonies of other corals (Figure 2A).

2) Rudimentary patch reefs dominated by $P$. damicornis with few small scattered colonies of other corals, particularly around the periphery (Figure 2B).

3) Rudimentary fringing reefs dominated by tabular and ramose Acropora species (Figure $2 \mathrm{C})$.

4) Mixed coral assemblages:

a) on rudimentary fringing reefs structurally dominated by Porites (Figure 3A);

b) on rudimentary fringing reefs with a largely obscured underlying framework showing patchy distribution of corals (Figure 3B); and

c) covering from $10-99 \%$ of the underlying baserock (Figures 3C, 3D).

5) Talus banks formed of fragments of dead branching corals (a) with little or no recolonization, (b) in the process of recolonization by the same species, (c) in the process of recolonization by different species.

6) Alcyonarians covering rock or dead coral colonies.

It is possible to separate these six coral community types visually based on conspicuous predominance of one or another coral (types 1,2,3,6) that, until closer scrutiny, appears to be the sole genus present. Types 4 and 5 are also clearly visually distinguishable: the former because of the conspicuous abundance of different corals, which is borne out by the rapid yield of coral genera per unit search time (Figures 3A-D); and the latter because of the conspicuous abundance of talus and dead coral colonies.

The genus-discovery curves (G-D curves) for the monogeneric rudimentary reefs level 
off rapidly and at low total yield of coral genera (Figure 2A-C). It is interesting that mixed coral assemblages yield similar G-D curves irrespective of the underlying substrate, whether Porites reef (Figure 3A), largely obscured reef framework (Figure 3B), or baserock (Figure 3C).

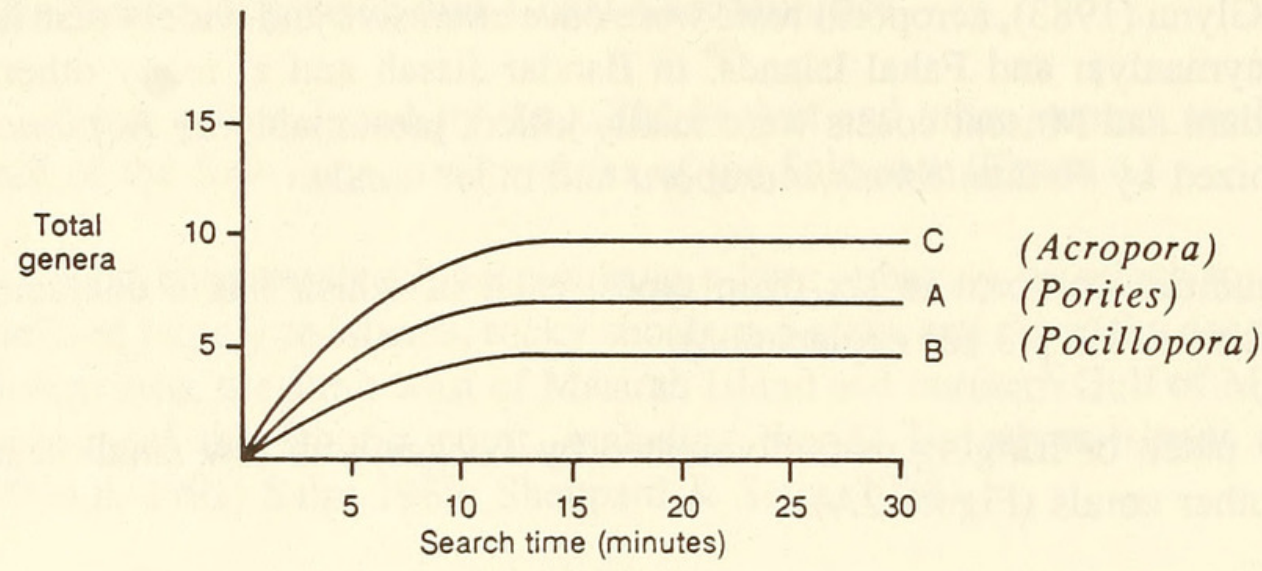

Figure 2. Genus-discovery curves for corals on "monogeneric" reefs.

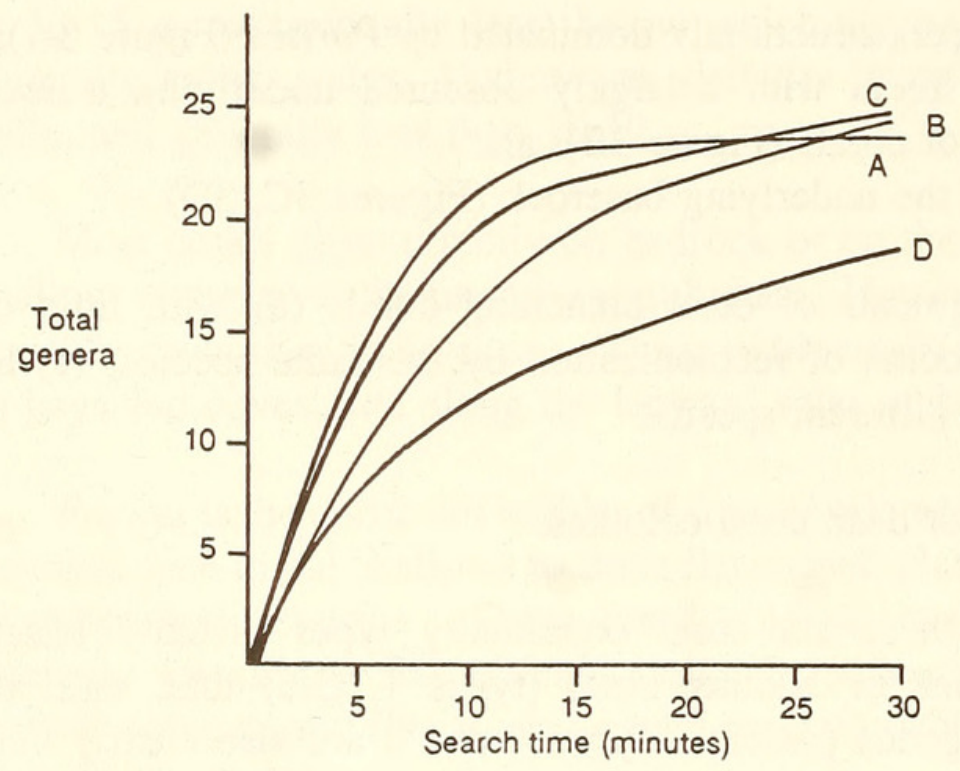

Figure 3. Genus-discovery curves for corals in mixed assemblages ( $\mathrm{A}=$ Porites framework obvious; $\mathrm{B}=$ obscured reef framework; $\mathrm{C}=\%$ cover of baserock $>60 \%$; $\mathrm{D}=$ $\%$ cover of baserock $<30 \%$ ). 


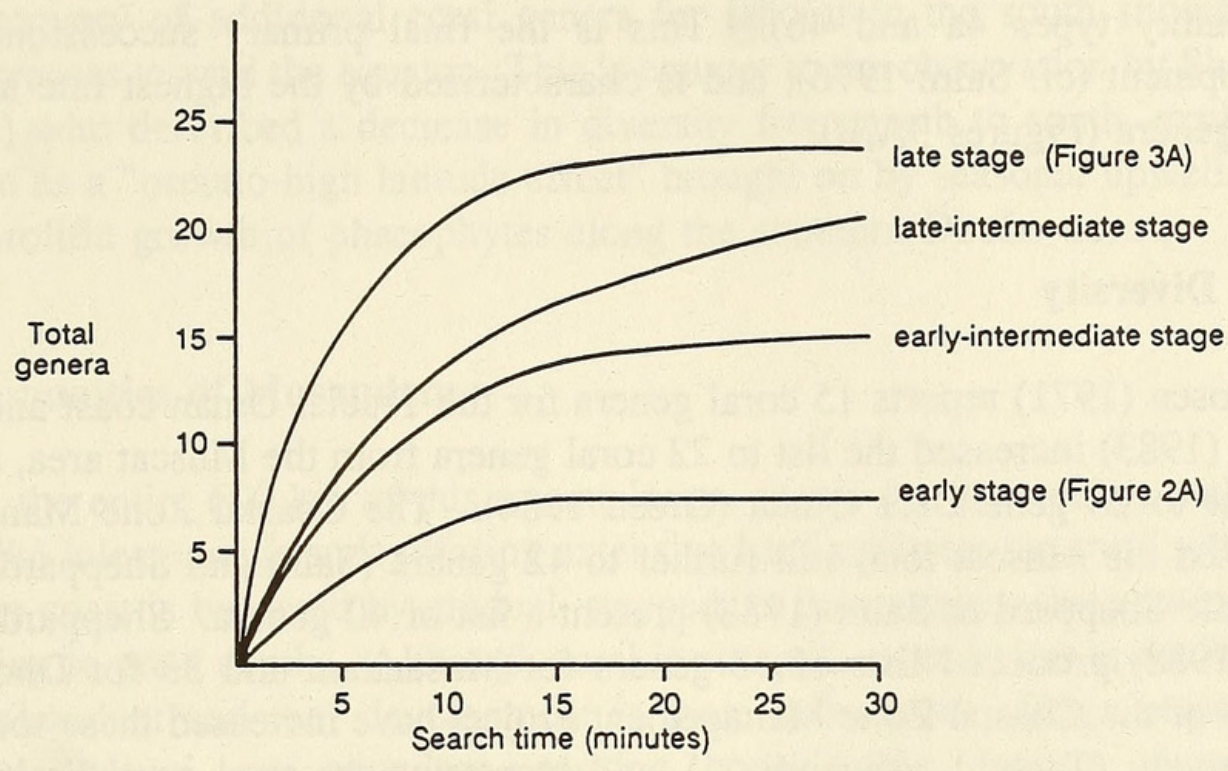

Figure 4. Genus-discovery curves for corals in the primary successional stages of reef development.

The G-D curves support another intuitively obvious observation: mixed coral assemblages with a high percentage cover (more than $60 \%$ ) yield genera more rapidly per unit search time than those with less than $30 \%$ cover (Figures $3 \mathrm{C}$ and 3D, respectively). Thus the G-D curve method of recording and displaying coral survey data offers a useful rapid survey tool for classifying coral communities. Its application in this respect merits further investigation.

There is a continuum in G-D curves between those for Porites dominated communities (Figure 2A) and mixed assemblage communities (Figure 3). These represent the different stages of reef development. For example:

Early stages of reef development conform to a type 1 community where the substrate is colonized by juxtaposed Porites colonies in different stages of fusion with adjacent colonies. This is characterized by a G-D curve with a low rate and total yield of coral genera (Figure 2A).

Intermediate stages of reef development that have Porites as the conspicuous structurally dominant coral, but adjacent colonies are fused, flat-topped and dead centrally (micro-atoll form), and colonized by an intermediate variety of corals. These are characterized by G-D curves with genus yield rates and totals intermediate between those 
for coral community types 1 and $4 a$ (Figure 4).

Late stages of reef development in which the Porites matrix is weakened, colonies die and/or cleave apart providing new hard substrate for colonization by corals (coral community types $4 \mathrm{a}$ and $4 \mathrm{~b}$ ). This is the final primary successional stage of reef development (cf. Salm 1976), and is characterized by the highest rate and total yield of coral genera (Figures $3 \mathrm{~A}, \mathrm{~B}$ ).

\section{Coral Diversity}

Rosen (1971) reports 15 coral genera for the Trucial Oman coast and Sawqirah Bay. Green (1983) increased the list to 22 coral genera from the Muscat area, and later revised this list to 28 genera for Oman (Green 1984). The Coastal Zone Management Project increased the Muscat total still further to 42 genera (Salm and Sheppard 1986 Appendix 1), while Sheppard \& Salm (1988) present a list of 40 genera. Sheppard (in Sheppard \& Salm 1988) produced lists of 38 genera for Musandam and 33 for Dhofar. Additional studies of the Coastal Zone Management Project have increased these totals to 41 and 48 respectively (Table 1, Appendix 1), and increasing the total coral list for the Sultanate from 47 to 53 genera, including the subgenus Porites (Synaraea), making the list complete or very nearly so (Appendix 1).

However, I have been shown a fungiid coral resembling Cycloseris that was allegedly collected from the Raaha area of Dhofar and have been told of other possible sightings, all unconfirmed. Also, two coral genera remain unidentified. Study of the many coral specimens deposited with the Oman Natural History Museum by a coral taxonomist would yield a detailed list of species, possibly including some new to science.

The Fahal Island area has the highest coral diversity (41 recorded genera) of all sites studied in the Sultanate, with all but one genus (Stylocoeniella) known from the Muscat area found there. The variety of substrate, depths and exposures to waves and currents in the vicinity of the island are the principal determinants of this high variety of corals, with the deeper rocky outcrops (25-42 m) contributing six additional corals to the total. This is also the type location for the new species Acanthastrea maxima Sheppard and Salm 1988, and the site I also first discovered Parasimplastrea simplicitexta, a species hitherto only known from fossil specimens from which it was originally described as Goniastrea simplicitexta Umbgrove (Sheppard \& Salm 1988). However, this new genus is clearly not Goniastrea and is closest to Simplastrea Umbgrove, hence the derivation of the name Parasimplastrea.

As might be expected, coral diversity increases south toward the equator from Musandam (41 genera), which is equivalent to the Muscat area (42 genera) although the composition is a little different, to Dhofar (48 genera). The diversity of corals in the northern Gulf of Masirah is low (27 genera) which probably reflects the isolation of this "island" of coral along a long largely coral-free coast. 
The Wildlife and Conservation Area Management Project of the Ministry of Environment has studied corals around Masirah in detail, and likely will increase the number of coral genera for the area. The results of this study are currently not available.

The discovery of additional coral genera for Dhofar in the south shows that coral diversity increases toward the equator. This is counter to the observation by Sheppard and Salm (1988) who described a decrease in diversity from north to south, explaining this phenomenon as a "pseudo-high latitude effect" brought on by seasonal upwelling of cold water and prolific growth of phaeophytes along the southern Dhofar coast.

\section{Coral Communities of Musandam}

Almost the entire $600 \mathrm{~km}$ of this mountainous, scenic coast is rocky, with its coves, bays, fjordlike inlets, and islands offering extensive hard substrate for coral settlement and growth. The coast is bordered by gradual, stepped, or increasingly steep rocky slopes, or cliffs plunging to great depths. Although coral is not quite as varied as in the Muscat area, it is more abundant and reef development is more substantial. Huge crown-of-thorns starfishes, $A$. planci, are abundant, causing considerable damage to many coral communities of the east and north coasts.

Musandam is formed of two principal components. The Ru'us al Jibal to the south is a prominent mountain complex that rises abruptly from the low sandy plains of the United Arab Emirates. It is joined by a narrow isthmus to the Musandam Peninsula which extends north into the Strait of Hormuz. The Musandam Peninsula is formed of a series of precipitous peninsulas and headlands enclosing deep fjordlike inlets.

The Ru'us al Jibal is generally bordered by shallow sandy seabed along the southeast coast in the Gulf of Oman and the Arabian Gulf coast to the west. Sandy beaches are largely confined to these coasts. 
Table 1. List of coral genera by region of Oman

Family Genus Musandam Muscat Masirah Gulf

Dhofar

\begin{tabular}{|c|c|}
\hline Thamnasteriidae & Psammocora \\
\hline Astrocoeniidae & Stylocoeniella \\
\hline \multirow[t]{4}{*}{ Pocilloporidae } & Pocillopora \\
\hline & Stylophora \\
\hline & Seriatopora \\
\hline & Madracis \\
\hline \multirow[t]{3}{*}{ Acroporidae } & Astreopora \\
\hline & Acropora \\
\hline & Montipora \\
\hline \multirow[t]{3}{*}{ Agariciidae } & Pavona \\
\hline & Leptoseris \\
\hline & Gardineroseris \\
\hline \multirow[t]{4}{*}{ Siderastreidae } & Siderastrea \\
\hline & Pseudosiderastrea \\
\hline & Anomastrea \\
\hline & Coscinaraea \\
\hline \multirow[t]{4}{*}{ Poritidae } & Porites \\
\hline & Porites (Synaraea) \\
\hline & Goniopora \\
\hline & Alveopora \\
\hline \multirow[t]{10}{*}{ Faviidae } & Favia \\
\hline & Favites \\
\hline & Goniastrea \\
\hline & Platygyra \\
\hline & Leptoria \\
\hline & Montastrea \\
\hline & Plesiastrea \\
\hline & Leptastrea \\
\hline & Cyphastrea \\
\hline & Echinopora \\
\hline \multirow[t]{2}{*}{ Rhizangidae } & Culicia $^{1}$ \\
\hline & Phyllangia ${ }^{1}$ \\
\hline \multirow[t]{2}{*}{ Oculinidae } & Galaxea \\
\hline & Parasimplastrea \\
\hline Merulinidae & Hydnophora \\
\hline \multirow[t]{3}{*}{ Mussidae } & Blastomussa \\
\hline & Symphyllia \\
\hline & Acanthastrea \\
\hline \multirow[t]{2}{*}{ Pectiniidae } & Echinophyllia \\
\hline & Oxypora \\
\hline \multirow[t]{5}{*}{ Caryophylliidae } & Euphyllia \\
\hline & Paracyathus \\
\hline & Polycyathus ${ }^{1}$ \\
\hline & Heterocyathus \\
\hline & Caryophylliid $^{1}$ \\
\hline
\end{tabular}

$\mathrm{x}$
$\mathrm{x}$
$\mathrm{x}$
$\mathrm{x}$
$\mathrm{x}$
$\mathrm{x}$
$\mathrm{x}$
$\mathrm{x}$
$\mathrm{x}$

$\mathrm{x} \quad \mathrm{x}$

$x-x$

$\mathrm{x} \quad \mathrm{x}$

$\mathrm{x} \quad \mathrm{x}$

$x$

$\mathrm{x} \quad \mathrm{x}$

$x \quad x$

$x-x$

$\mathrm{x} \quad \mathrm{x}$

$\mathrm{x}$

$\mathrm{x}$

$\mathrm{x}$

$\mathrm{x}$

$\mathrm{x}$

$\mathrm{x}$

$\mathrm{x}$

$\mathrm{x}$

$\mathbf{x}$

$\mathrm{x}$

$\mathrm{x}$

$\mathrm{x}$

$\mathrm{x}$

$\mathrm{x}$

$\mathrm{x}$

$x$

$\mathrm{x}$

$\mathrm{x}$

$x$

$\mathrm{x}$

$\mathrm{x}$

$\mathrm{x}$

$\mathrm{x}$

$\mathrm{x}$

$\mathrm{x}$

$\mathrm{x}$

$\mathrm{x}$

$\mathrm{x}$ $\mathbf{x}$

$\mathbf{x}$

*

$\mathbf{x}$

x

$\mathrm{X}$

$\mathrm{X}$

$\mathrm{X}$

X

$\mathrm{x}$

$\mathrm{X}$

$\mathrm{X}$

$\mathrm{X}$

$\mathrm{x}$

$\mathrm{x}$

X

$\mathrm{x}$

$\mathrm{x}$

$\mathrm{x}$

$\mathrm{x}$

$\mathrm{x}$

X

$\mathrm{x}$

$\mathrm{x}$

X

$\mathrm{x}$

X

X

$\mathrm{x}$

X

$\mathrm{x}$

$\mathrm{x}$

$\mathrm{x}$

x

$\mathrm{x}$

X

$\mathrm{x}$

X

X 


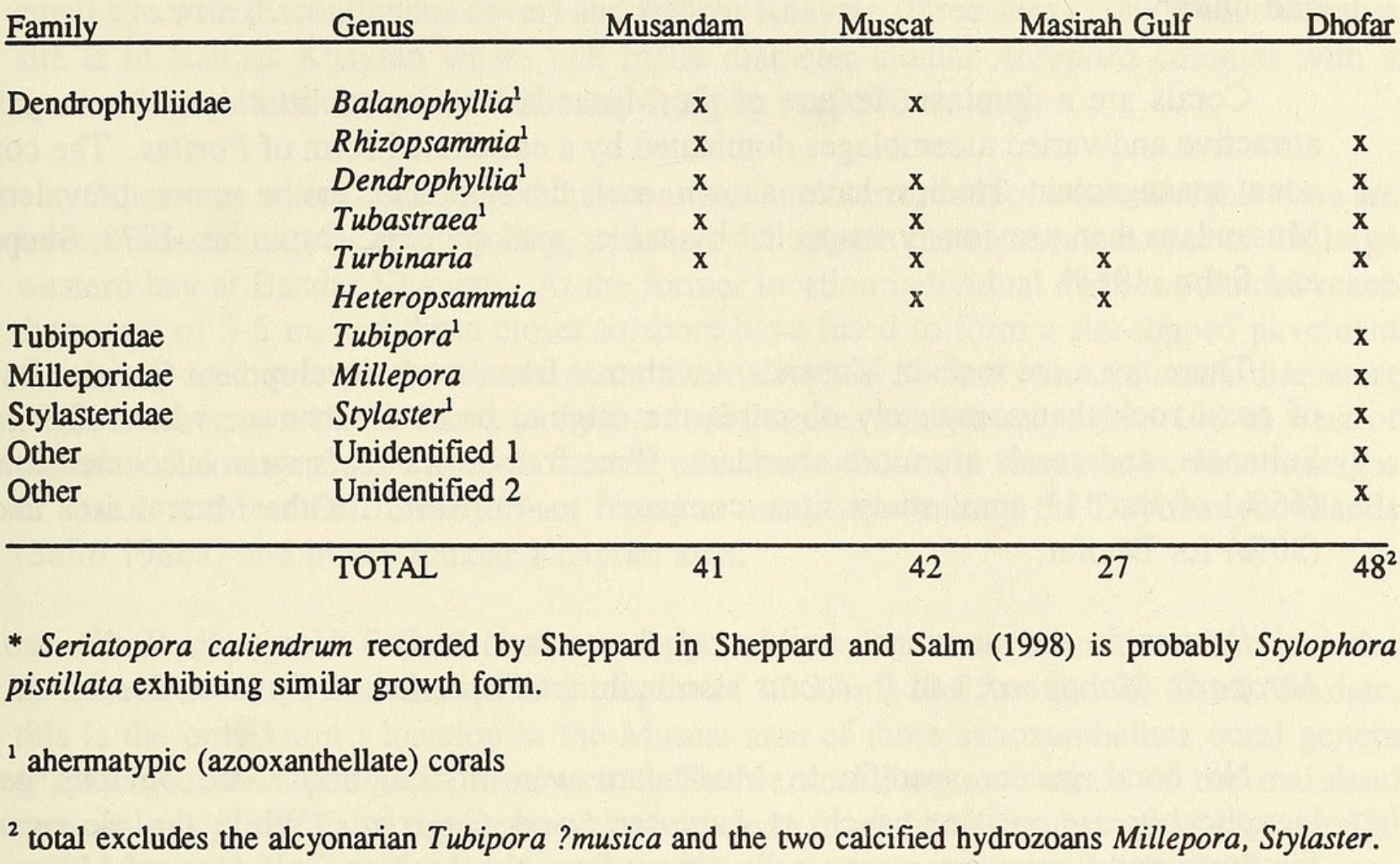

The coast along the northeast of the Ru'us al Jibal and the Musandam Peninsula falls away rapidly to great depths, often as sheer underwater cliffs. Gravel or cobble beaches are common here, and sandy beaches are few and small. Strong tidal currents scour this coast and eddy in many of the small coves. These current eddies concentrate flotsam and the larvae of marine creatures, both of which have a significant impact on benthic communities.

Limestone cliffs, reaching heights of over $240 \mathrm{~m}$ in places, are a major structural component of the coastline covered by this plan, and form $66 \%(396 \mathrm{~km})$ of its total length. The cliffs are deeply eroded in the intertidal by the combined action of boring organisms (notably the mussel Lithophaga) and current scour.

Along most of their length, the cliffs are bordered by seas deeper than six metres with scattered rocky reefs. Elsewhere, the cliffs fall to shallower seas, or an intertidal sand or rock shelf. The well-shaded cliffs suit the growth of flattened sheets of corals that commonly encrust these sheer walls. Among these, Pectinid corals (Oxypora lacera, Echinophyllia aspera) and Echinopora spp. are well represented and, upon closer examination, may yield additional species for Oman.

Rocky shores dominate the coasts bordering wadi fans and beaches in the inner reaches of the fjordlike inlets and other bays, forming about $28.5 \%$ of its total length. 
These are formed of rock falls, eroded mountain slopes, beach concretions, and wadi fan conglomerates. These rocky shores generally extend underwater as rocky slopes, terraces and outcrops.

Corals are a dominant feature of the Musandam rocky sublittoral, where they form attractive and varied assemblages dominated by a castellated form.of Porites. The coastal zone management studies have shown reef development to be more prevalent in Musandam than previously suspected by earlier workers (e.g., Cornelius, 1973; Sheppard and Salm, 1988).

There are more reefs in Musandam with true framework development (i.e., a substrate of coral rock that completely obscures the original bedrock) than anywhere else in the Sultanate, and corals are more abundant. True framework reefs were encountered at 73 (66\%) of the 111 coral study sites, compared to $43(61 \%)$ for the Muscat area and 17 (30\%) for Dhofar.

Porites is the dominant builder of framework reefs. However, P. damicornis, Acropora, Montipora, and $P$. cactus also build small patches of framework reef.

No coral genera specific to Musandam were found, and some obvious genera inexplicably are missing, such as Leptoseris and Galaxea. While the alcyonarians Sinularia and Sarcophyton are totally absent from the Arabian Gulf coast of Musandam, and surprisingly sparse elsewhere along most of the rest of the Musandam coast, the brilliant red, orange, yellow or mauve Dendronephthya klunzingeri, purple and bushy orange gorgonians, and bright yellow Antipathes sp. are notably abundant.

Four coral species that are well represented in Musandam waters are not found elsewhere in the Sultanate. These, which are yet to be identified, include a form of $P a v o n a$ similar to $P$. cactus but with extended leaves resembling $P$. decussata; a finebranched Acropora that forms extensive tangled thickets; a castellated species of Porites that is covered by tapering spires; and a "tree"-forming type of Tubastraea, sometimes found in very shallow water where the current runs strong.

\section{Coral Communities of the Muscat Area}

The exposed north and east facing coasts tend to support relatively small scattered scleractinian coral colonies, and more extensive patches of alcyonarians, chiefly Sarcophyton. Percentage cover of living scleractinian corals rarely exceeds $5 \%$ of the substrate in these locations.

The leeward west-facing coasts of headlands, southern island shores, and sheltered locations favour the growth of dense coral assemblages (percent cover up to 99\%) and framework reefs. 
Despite the extensive die-off of acroporid reefs in the Muscat area a number of years ago, there are unaffected Acropora communities in two sheltered bays: Bandar Jissah (one small site with discontinuous cover) and Bandar Khayran (three sites). The most extensive site is in Bandar Khayran where two metre diameter tabular Acropora colonies with a cover of up to $99 \%$ form a rudimentary fringing reef $200 \mathrm{~m}$ long.

Other sites where coral growth has continued uninterrupted for a considerable time are the southeast cove of Cemetery Bay, and the small islet in the southeast corner of the large western bay at Bandar Khayran. At the former location individual Porites colonies reach diameters of 3-5 m, and those closer to shore have fused to form a flat-topped pavement that is exposed at low tide. At the latter site individual Porites colonies reach diameters of three metres and have fused to form a reef-flat that extends more than $100 \mathrm{~m}$ west of the islet. "Younger" Porites reefs formed of colonies 2-3 $\mathrm{m}$ in diameter in various stages of fusion and reef-flat formation are found at many sites among the Daymaniyat islands (Salm 1986a) and in the Bandar Khayran area.

Three sites in the Fahal Island area support unusual coral communities. One includes three rock outcrops near the island in depths of $17-25 \mathrm{~m}, 20-35 \mathrm{~m}$ and $22-42 \mathrm{~m}$. To date, this is the only known location in the Muscat area of three azooxanthellate coral genera (Phyllangia, Rhizopsammia, Madracis). The vertical limestone strata of the mainland peninsula opposite Fahal dip seaward and protrude from the silty substrate as low parallel underwater ridges, forming another site. Where they emerge at a depth of $15 \mathrm{~m}$, they support a number of colonies of the newly described species Acanthastrea maxima (Sheppard \& Salm 1988). Although isolated colonies of this coral occur elsewhere in Oman, this is the only place where they occur grouped and attain such large calyx (up to $60 \mathrm{~mm})$ and polyp size $(80-100 \mathrm{~mm})$.

The silty substrate between Fahal Island and the mainland is the third site and only location so far that Heteropsammia cochlea has been found in Oman. Heterocyathus aequicostatus probably also occurs here, but so far has only been found on beaches of the Batinah and central Oman coasts.

\section{Coral Communities of the Gulf of Masirah Area}

The west coast of Masirah Island and the Barr al Hikman, separated by a strait to the west, have well developed coral reefs dominated by Platygyra and M. foliosa. The diversity of corals here is not high, reaching only 27 genera around Barr al Hikman compared to 41 for Musandam, 42 for the Muscat area, and 51 for Dhofar. Between 750 $\mathrm{m}$ and $2.3 \mathrm{~km}$ off the south coast of Barr al Hikman, and protected by their inaccessibility, lie the largest true coral reefs in the Sultanate. Some of these reefs, which measure many square kilometres, are of further interest because they are built exclusively of $M$. foliosa. Elsewhere in Oman there are few well developed examples of coral reefs, and these are generally small. Coral assemblages covering underlying rock without development of true reef framework are more common. The reefs south of Barr al Hikman form one of the 
living natural spectacles of Oman.

Glynn (1987) notes that there are no Acanthaster around Masirah Island, suggesting that consequent lack of predation enables the fast growing Montipora foliosa to dominate other corals by competitive overtopping, and form large monogeneric stands. During numerous days of study over a six year period, no Acanthaster were found on the reefs fringing Barr al Hikman opposite Masirah Island, which also may explain the proliferation of Montipora foliosa there into the huge reefs described above.

These remote reefs are unique in the Sultanate from another perspective: the Hikmani tribe who have traditional fishing rights to the area, prohibit all fishing on the reefs for commercial purposes. Fishing is permitted during times of adversity, but for domestic use only. This is a self-enforced community doctrine which effectively maintains the reef as a living cold store.

These hitherto unstudied reefs have many rare and little known species of fishes, and have yielded at least one species new to science: the butterflyfish Chaetodon dialeucos (Salm \& Mee, 1989).

\section{Coral Communities of Dhofar}

The mainland coast of Dhofar is approximately $800 \mathrm{~km}$ long, stretching from Wadi Haytam on the Jazir coast in the north to Yemen in the south. It includes cliff, rocky and sandy shores. There are numerous small rocky islets close to shore, and the Al Halaniyat Islands (until recently called the Kuria Muria Islands) - the largest group of offshore islands in the Sultanate. Almost the entire coast is seasonally exposed to wave action, ranging from moderate to heavy. Sheltered shores are found in the many coves and larger inlets along the rocky embayed coast between Hasik and the Yemen border.

During summer months, upwellings fuel dense growth of phaeophytes that reach over one metre in length in approximately four months. In the north and central parts there is a mix of Sargassopsis zamadinii and Sargassum spp., that reach from the intertidal to a depth of about $10 \mathrm{~m}$. In the central part, the kelp Ecklonia radiata predominates below this zone to a depth of about 12-15 m. In the south Sargassum alone is dominant down to below $12 \mathrm{~m}$. The splash zone of the entire exposed coast is dominated by Ulva. These algal communities have been studied in some detail (Barratt 1984). Dhofar is unusual in having dense coral and algal communities growing seasonally in juxtaposition or even in mixed assemblages.

Limestone cliffs are a major structural component of the Dhofar coastline, forming about $25 \%$ of its total length. Along most of their length, the cliffs are bordered by seas deeper than six metres with scattered rocky reefs. Elsewhere, the cliffs fall to shallower seas, or to an intertidal sand or rock shelf. 
Rocky shores dominate the coast from Hasik to Marbat, and from Mughsayl to the Yemen border, forming about $30 \%$ of its total length, and around most of the Al Halaniyat Islands. Rocky reefs fringe most of this coast, providing a substrate for coral and algal communities. The more exposed parts of this coast are fringed by rock pavement covered by algal turf. Algal turfs also carpet many other rocky reefs. These turfs comprise a mixture of different filamentous and smaller algae, and show marked seasonal responses to oceanographic conditions. They may be totally dominated and obscured by macroalgae during the summer season of upwelling. These turf-covered rock pavements are feeding grounds for green and loggerhead turtles.

The sheltered mainland coves and Al Halaniyat Islands support dense coral assemblages and limited framework reefs, growing in unusual association with dense algal growth during the summer monsoon.

The living coral cover generally ranges between less than $0.5 \%$ to $80 \%$ of the substrate, with levels up to $99 \%$ on the reef formations east of Marbat and around the $\mathrm{Al}$ Halaniyat Islands. The coastal zone management studies have increased the number of corals known from Dhofar by at least $45 \%$ from 33 to 48 coral genera, and have yielded four new records of scleractinian corals for Oman (Goniastrea pectinata, Montastrea curta and two unidentified genera), and two calcareous species, the alcyonarian Tubipora ?musica and the hydrozoan Millepora. In addition, true reef development has been documented at 15 new sites, bringing the total of known framework reefs in Dhofar to 17. Porites provides the framework for these reefs, which may reach a thickness of three metres. One Porites colony measured $12 \mathrm{~m}$ in diameter, probably representing more than 1,000 years growth, and is the largest such colony so far discovered in the Sultanate.

Porites supports dense seasonal breeding concentrations of the spiny lobster Panulirus homarus in at least four sites, and shallow rocky reefs attract smaller concentrations in at least two sites.

Certain of the Dhofar corals, fishes, seaweeds, starfishes, and sea urchins are not found elsewhere in Oman. For example, the coral communities include at least five scleractinian genera (Euphyllia fimbriata, G. pectinata, M. curta, Leptoria phrygia, Gardineroseris planulata), one alcyonarian genus (Tubipora), and two hydrozoan genera (Millepora, Stylaster) that are found only in Dhofar, and the fishes include at least three butterflyfishes (Chaetodon lunula, Chaetodon auriga, Megaprotodon strigangulus), and the newly described Amphiprion omanensis Allen \& Mee 1991, that are absent or uncommon elsewhere in Oman.

Sandy beaches are a major component of the Dhofar coast, and occupy $40 \%$ of its total length, while gravel/cobble beaches form about $5 \%$. Small ephemeral beaches form and erode along parts of the coast. The seabed off the beaches is shallow and sandy with flat rocky reefs subject to heavy sand scour during the rough summer monsoon. 


\section{THREATS TO CORAL COMMUNITIES}

Reef growth is interrupted in Oman by episodic coral mortality. This appears to be confined to corals in more exposed locations. But the pattern of die-off is confusing, as the damage may affect only parts of a reef or one in a series of adjacent coral patches.

All that remains of former reef development at the die-off sites are large dead Porites, Platygyra or Symphyllia colonies, the stumps of once large tabular Acropora, or talus banks formed of dead P. damicornis or Acropora.

Large patches of dead coral are common in the Muscat area and Musandam, but not the Masirah area or Dhofar. Some areas have been killed by the crown-of-thorns starfish $A$. planci, others by entangling fishing nets, and damage to corals by the anchors of fishermen and divers remains a problem on many reefs.

A white patch disease, where necrotic coral tissue sloughs rapidly off colonies of Platygyra exposing the white skeleton beneath, is prevalent in parts of Musandam, and appears to be a significant cause of mortality for the genus. No invasion of coral tissue by pathogens is visible in situ, and tissue specimens were not collected for further study.

Collapse of coastal limestone cliffs undercut by the boring mussel Lithophaga and wave scour destroys coral colonies, as noted by Glynn (1983). However, this phenomenon does not explain die-offs in areas far from cliffs or signs of recent rock falls, such as along the southern shores of the Daymaniyat Islands.

Episodic massive discharge of silt-laden flood waters into the sea certainly would destroy corals in affected areas. However, this phenomenon is more likely to explain the absence of reef formations than the die-off patterns. It is unlikely to have any effect on corals of the Daymaniyat Islands which lie $18-20 \mathrm{~km}$ offshore.

Temperature stress is another likely cause of coral mortality and its effect on reef development in Oman requires study. The sudden chilling of surface water during summer is well-known among the diving community. Summer is a period of unpredictable and localized upwelling in the Muscat area. Cells of cold upwelling water drawn by winds or currents over a coral community would cause a precipitous drop in temperature of many degrees centigrade. Green (pers. comm.) has noted a drop in surface seawater temperature of $15^{\circ} \mathrm{C}$ over 14 days in July 1983 , and regular monthly fluctuations of as much as $8^{\circ} \mathrm{C}$ during summer (Green 1983). Cold water upwelling is known to cause coral bleaching and mortality, with areas prone to these events having few, small and young reefs (Glynn, 1990) - a condition that fits coral communities of the Sultanate. If temperature stress proves to be a cause of coral mortality, it would help explain the disjunct pattern of dieoff, death of large Porites and Symphyllia colonies, and dead corals in areas swept by currents near deep water. 
Temperature-induced bleaching and mortality of corals is a definite problem. Coral bleaching in the summer of 1990 caused some corals to die in the Muscat area, but killed up to $95 \%$ of the corals shallower than three metres on reefs in Musandam.

The pattern of coral community development and die-off is a product of all above parameters. Recreational diving is a more recent cause of coral damage (Salm 1986b), but its effects are currently localized and relatively slight.

Of the 239 coral communities described for Musandam, Muscat and Dhofar (Appendix 2), at least $162(68 \%)$ are damaged by fishery activities, litter and A. planci predation. Musandam has the highest incidence of damage ( 93 sites, $84 \%$ of the study sites in Musandam), followed by the Muscat area with 48 sites (68\% of study sites there), and Dhofar (24 sites, 37\% of total Dhofar sites).

Bioerosion of damaged or dead corals has a serious impact on development of reef framework, and follows the pattern described by Glynn (1988) in the eastern Pacific. The principal agents of bioerosion include boring algae, sponges, the mussel Lithophaga, and the echinoids Diadema setosum, Echinothrix diadema, and Echinometra mathaei. Diadema forms the densest accumulations, massing on talus areas around the bases of live coral colonies.

\section{Fishery Related Damage to Coral Communities}

There is a considerable amount of dead and broken coral in Musandam and the Muscat area. Fishing activity certainly contributes to this. Gill nets, fish traps, and anchors are dropped directly onto reefs of branching coral species, breaking them, and frequently becoming entangled. If these cannot be broken free, they are abandoned in situ, and contribute to further coral damage. Monofilament net, the use of which is prohibited in Oman, is especially damaging to marine life when abandoned on the seabed. Use of monofilament nets is increasing in the Sultanate, and few villages are without them. One such net examined in Musandam yielded a number of live fishes, crabs and lobsters among many more that were dead. Ropes, gill nets and fish traps entangled in and damaging corals were recorded at $60(54 \%)$ sites studied underwater in Musandam, $43(61 \%)$ in the Muscat area and, 20 (35\%) in Dhofar.

Patches of $P$. damicornis and Acropora spp. are particularly vulnerable to damage from abandoned fishing nets. The nets entangle and break off coral branches which roll up in them. A net weighted in this way and still partly supported by floats can bounce and drag over coral, leaving a swathe of dead rubble. Around the Daymaniyat Islands, 25$80 \%$ of some $P$. damicornis patches have been destroyed in this way. Although nets were found to kill part or all of the corals they entangled, this still would not explain the pattern of complete die-off, especially in areas lacking any sign of nets or physical damage to corals. 
Litter abounds underwater in Musandam. Plastic bags, fabric and the ballast bags of Iranian small boat traders are especially damaging to corals which they envelop and smother. In sheltered coves where fishermen careen their boats for maintenance work, sheets of linoleum and plastic, paint cans and other rubbish are snagged in corals. At least $12(30 \%)$ coral sites examined showed considerable damage attributable to fishermen and these traders; at one site alone, more than 120 pairs of trousers (part of an Iranian consignment) were seen caught in and smothering coral. Thirty-one (28\%) of the coral sites viewed suffered from litter damage caused by fishermen alone. Between their litter and fishery activity, fishermen caused damage to corals at $80(72 \%)$ of the 111 study sites in Musandam. The extent of pollution in this remote and beautiful area is stunning and tragic.

The quantity of litter underwater attributable to fishermen is far less in the Muscat area (7 sites, 10\%) and Dhofar (6 sites, 11\%) than Musandam. The combined effect of fishery activity and litter damages coral at 43 sites $(61 \%)$ in the Muscat area, and 20 sites (35\%) in Dhofar. There are many other sites in Oman where nets are entangled on the substrate, threatening benthic invertebrate communities, fishes, spiny lobsters, dolphins and turtles.

The Sultanate is concerned about the environment, but only now is becoming aware of the damage beneath the seas. Five years ago a large underwater clean-up was sponsored by the Ministry of Commerce and Industry through the Tourism Department. Eighty five divers took part in this two day event, pulling up more than three tons of old fishing nets. This has been followed by two recent clean-up campaigns inspired and organized by the Intercontinental Hotel in collaboration with the Ministry of Environment. These two clean-ups yielded tons more fishing nets and other rubbish from the seabed, and were well supported by many concerned divers. Among the victims dead in these nets were many crabs, fishes and lobsters, and one green turtle.

\section{Acanthaster planci}

The distribution of $A$. planci in Oman appears to be restricted to the Gulf of Oman and the Strait of Hormuz. During the course of the seven-year CZM study, none was found along the Arabian Sea or Arabian Gulf coasts of Oman. These observations agree with those of Glynn (1983) who carried out a dedicated survey for the species.

However, Glynn (1983) found no Acanthaster in 12 sample sites in Musandam that he visited in 1982. Eleven of these sites were along the Arabian Gulf coast where the CZM studies showed the starfish to be absent in 1989/90, and one site was in Khawr Kumzar in the north where there was a profusion of Acanthaster in 1989/90. Because of the localized nature of the crown-of-thorns outbreaks, it is possible that Glynn simply missed the starfish in Khawr Kumzar, if indeed they were there.

A. planci is a major cause of coral destruction in Musandam. Severe infestations 
associated with large patches of dead coral where found at $57(51 \%)$ of the locations studied underwater by the CZM project, all on the north and east coasts. No Acanthaster were found on the west coast. The largest infestation was found off Jazirat Habalayn where 117 starfishes were counted in 15 minutes off the south side of the island. Sheppard (1986) makes no mention of Acanthaster at this site four years previously.

Also in 1986, Zahuriyin in Khawr Habalayn had a new infestation in an area of luxuriant table coral Acropora, with $27 \mathrm{~A}$. planci seen exposed in 0.4 hectare (Salm, 1986c). Based on a visual estimate, the starfishes had killed $25 \%$ of the Acropora community. By 1990, this same site was reduced to a wasteland of dead and broken coral with very limited recolonization along the deep edge of the former coral band.

Currents eddy in the many small coves along the mountainous Musandam coast and around islands, concentrating flotsam which eventually sinks and explains the abundance of litter underwater there. These current eddies also would concentrate coral and A. planci larvae (hence better coral settlement and growth and higher coral predation there), helping to explain the numerous, small, disjunct concentrations of $A$. planci.

Many of these coves are found below steep valleys at the confluences of cliffs or precipitous mountain faces. Rainwater and mud runoff at these sites is high and concentrated by eddies which heighten their effect on corals. Perhaps this localized runoff effect and high $A$. planci predation combine to cause episodic mass mortality of vulnerable corals and to explain the high incidence of dead coral and talus banks in many coves.

Table 2. Corals eaten by Acanthaster planci in Oman

\author{
Psammocora sp. \\ Stylophora pistillata \\ Pocillopora damicornis \\ Acropora spp. \\ Montipora spp. \\ Porites spp. \\ Goniopora sp. \\ Pavona cactus \\ Platygyra daedalea \\ Cyphastrea serailia \\ Echinophyllia aspera
}


Glynn (1983) concludes that recurring outbreaks of crown-of-thorns starfish, A.planci, may have a large influence on the development of coral communities in the Muscat area. However, as Glynn points out, Acanthaster is a fairly selective feeder, which generally avoids colonies of Pocillopora, Porites and Symphyllia (although it regularly does feed on both Pocillopora and Porites in Oman). Thus, it is unlikely that starfish predation alone would cause the extensive mortality of large colonies or patches of these three corals.

In Oman, A. planci was seen to feed on a variety of hard corals (Table 2) and the alcyonarian Dendronephthya klunzingeri, but never Symphyllia, and clearly preferred species of Acropora.

Glynn (1987) notes that commensal crustacean guards render pocilloporid reefs apparently resistant to Acanthaster predation, even when at outbreak densities, which may explain the prevalence of Pocillopora patches in many areas where other corals are largely dead.

A. planci was relatively common in the Muscat area, being seen at $20(28 \%)$ study sites, but never in concentrations equivalent to those of Musandam. The vast coral-free sandy coast at Ra's al Hadd (the breakpoint between the Gulf of Oman and the Arabian Sea), and the pattern of northerly currents sweeping up to this point from the south with easterly currents moving offshore to the north, possibly prevent the southerly drift of $A$. planci larvae and its invasion of Arabian Sea coral communities from the north. The pattern of seasonal upwelling and low seawater temperatures $\left(16-19^{\circ} \mathrm{C}\right)$ along the south coast of Oman may well prevent invasion from that direction, as cool water is known to inhibit development of Acanthaster larvae (Glynn 1983).

\section{Coral Bleaching in the Sultanate of Oman}

Coral bleaching is the loss of colour that accompanies an expulsion of the symbiotic zooxanthellae (microscopic plankton living in the coral tissue). It was described by Vaughan as long ago as 1914, and was first recognized to result from elevated seawater temperatures by Yonge and Nichols (1931). Recently, and undoubtedly in response to increasing concern among scientists about global warming, coral bleaching has been the focus of considerable field and laboratory study, leading to the general conclusion that elevated sea water temperatures coupled with UV radiation are the chief agents responsible for coral bleaching (cf. Brown 1990).

Coral bleaching in the Sultanate of Oman was noticed first by divers who expressed concern that corals were "dying" on the reefs off Marjan Beach, the public beach at Ra's al Hamra. Subsequent investigation of the area showed that there had been extensive bleaching of corals there, giving the impression that the corals were in fact dead.

Certain interested divers were briefed on coral bleaching and asked to report their observations. It was clear that bleaching was widespread throughout the Muscat area, 
where sea water temperatures had reached as high as $39^{\circ} \mathrm{C}$ in June 1990.

Field work undertaken through the IUCN Coastal Zone Management Project, was extended to investigate coral bleaching in Musandam, certain reefs being monitored from June through October 1990.

Coral bleaching and related mortality has not been recorded previously from the Sultanate of Oman. The amount of dead coral and lack of framework reef development, despite apparently suitable conditions, may well be attributable to past bleaching events.

\section{Coral Bleaching in the Muscat Area}

On 29 June 1990, sea water temperature at "Cat Island" off the Marine Science and Fisheries Centre reached $39^{\circ} \mathrm{C}$. There was reportedly extensive bleaching of corals surrounding the islet. When visited in September 1990, the corals had largely recovered. At that time the seawater temperature was $24^{\circ} \mathrm{C}$ down to a depth of four metres and up to $500 \mathrm{~m}$ off the island. Symphyllia was the only scleractinian coral to show residual signs of bleaching, whereas all colonies of the alcyonarian coral Sinularia in exposed locations remained bleached to a pale yellow colour.

The Marjan Beach area was first visited in July 1990 in response to reports of widespread coral mortality there. At that time the water temperature was $28^{\circ} \mathrm{C}$, and no mortality was recorded although many colonies of the following corals were severely bleached: Montipora and Porites, which are the most abundant corals, and Acropora which is less common in the area. The Acropora and some Montipora colonies were bleached pure white, and the Porites had turned very pale blue, mauve, brown or yellow.

Symphyllia, Favia, Hydnophora microconos, Turbinaria, Stylophora, Goniopora, and Acanthastrea echinata, were less severely bleached, retaining a yellow or yellow-brown colour, and all Platygyra were totally unaffected. The soft corals Sinularia and Sarcophyton, and great numbers of zoanthids also were severely bleached to very pale yellow. Many of the zoanthids were already dead, showing no reaction to touch and partly floating free.

The bleaching was confined to the inner reaches of the bay. By early September, the faviids, Porites, and Montipora had all regained normal colouration, and mortality appeared to be slight ( $<2 \%$ of all corals) and confined to colonies of Acropora.

Reports of extensive bleaching around Fahal Island and in Bandar Khayran were received from divers who regularly visit the areas. There was no sign of this bleaching by mid-October at Fahal Island, and there appeared to be no bleaching-related mortality. In early November, the only sign of bleaching at Bandar Khayran was pale yellow-green Sinularia in shallow exposed locations. 


\section{Coral Bleaching in Musandam}

In July 1990 two sites were investigated for coral bleaching: Jazirat Habalayn in Khawr Habalayn, and Jazirat Maqlab in Khawr Ash Shamm. The findings prompted follow-up monitoring of these sites in August and October, and the study of additional sites. Appendix 3 presents observations at these sites.

Coral bleaching was more severe in the large fjordlike bays than outside along open coasts. The temperatures were higher in these environments (e.g., August temperature ranges surface to $10 \mathrm{~m}$ : Khawr Habalayn $35-32^{\circ} \mathrm{C}$; Khawr Ash Shamm $34-33^{\circ} \mathrm{C}$ ) than in the well-flushed straits (Khawr Quway' $32-31^{\circ} \mathrm{C}$; Ra's Rarak in the southern Strait of Hormuz $32-30^{\circ} \mathrm{C}$ ) where there was no bleaching.

Bleaching and related mortality were most severe between the surface and three metres depth. Mortality varied from <1\% (Khawr Quway') to $>95 \%$ (Gulf of Oman coast, patches in Khawr Ash Shamm). The principal species affected were branching Acropora (Khawr Ash Shamm), Stylophora, Acropora and Platygyra (Khawr Habalayn), and table Acropora (Gulf of Oman coast).

Bleaching and related mortality may explain the enigma of the many hollowed-out live Platygyra daedalea colonies in the bays and fjords of Musandam. Observations at Maqaqah, following severe bleaching of Platygyra, show that dead patches are rapidly covered by silt and invaded by algae. This opens the way for numerous boring organisms to penetrate and hollow out the coral colony, including the conspicuous sea urchins Echinometra and Diadema. This rapid bioerosion of corals appears to be an important factor inhibiting the development of more framework reefs in Musandam.

Although the sea temperatures reached higher levels in the Muscat area, temperatures higher than $30^{\circ} \mathrm{C}$ were not sustained for as long as they were in Musandam. Sea temperatures in the Muscat area characteristically show great variability during summer, rising during periods of calm still weather and dropping rapidly in response to upwellings of cold water. Green and Keech (1986) report a drop in temperature at $10 \mathrm{~m}$ depth from $32^{\circ} \mathrm{C}$ to $17^{\circ} \mathrm{C}$ in less than 14 days in July 1983 . During 1990 , surface water temperatures dropped more rapidly, cooling from $39^{\circ} \mathrm{C}$ at the end of June to $24^{\circ} \mathrm{C}$ in less than one week. The Musandam sea temperatures reached $34-35^{\circ} \mathrm{C}$ and remained above $30^{\circ} \mathrm{C}$ down to a depth of $10 \mathrm{~m}$ for at least three months in the areas of greatest coral mortality.

Glynn et al. (1988) and Glynn (1990) report that the highest mean percent coral mortality in the eastern Pacific occurs in areas with normally lower and more variable sea water temperatures, and the lowest mortality occurs in areas with normally high and stable temperatures. However, the length of exposure to elevated temperatures is a key determinant of coral bleaching (Jokiel and Coles 1990) and, as the Oman examples show, of coral mortality. For example, the Muscat area, with greatly varying sea water temperatures, had shortest exposure to elevated temperatures and least coral mortality $(<2 \%)$. The high temperature $\left(39^{\circ} \mathrm{C}\right)$ was enough to induce bleaching, but the exposure 
apparently was too short to be lethal.

In Musandam, corals in straits with strong currents and well mixed cooler water experienced least mortality $(<1 \%)$, presumably because exposure to elevated temperatures was too short. It could be argued that the pattern observed by Glynn et al. (1988) and Glynn (1990) applies elsewhere in Musandam. For example, corals in the large fjordlike bays suffered lower mortality presumably because water circulation is lower and temperatures remain stable at higher levels than in the small open bays on the east coast where coral mortality in the upper three metres exceeded 95\%, and where presumably water circulation is greater, and temperatures generally lower and more variable. Unfortunately, baseline current and temperature data do not exist for these remote sites. 


\section{REFERENCES}

Barrat, L. 1984. Ecological study of the rocky shores on the south coast of Oman. IUCN report to UNEP Regional Seas Programme: 104 pp.

Brown, B.E.(ed.). 1990. Coral bleaching. Coral Reefs, 8: 153-232.

Burchard, J.E. 1980. Coral fauna of the western Arabian Gulf. Aramco, Dhahran, Saudi Arabia: $128 \mathrm{pp}$.

Cornelius, P.F.S. 1973. I. Biological aspects. In Cornelius, P.F.S., N.L. Falcon, D. Smith, C. Vita-Finzi. The Musandam Expedition 1971-72: Scientific Results, Part 1. Geogrl J. 139,3: 400-403.

Glynn, P.W. 1983. Final report on the effects of the sea star Acanthaster on Omani coral reefs. Ms. report to Ministry of Agriculture and Fisheries, Oman: 37 pp.

Glynn, P.W. 1987. Some ecological consequences of coral-crustacean guard mutualisms in the Indian and Pacific Oceans. Symbiosis, 4: 301-324.

Glynn, P.W. 1988. El Niño warming, coral mortality and reef framework destruction by echinoid bioerosion in the eastern Pacific. Galcaxea, 7: 129-160.

Glynn, P.W. 1989. Coral mortality and disturbances to coral reefs in the tropical eastern Pacific. In: Glynn, P.W.(ed.). Global ecological consequences of the 1982-83 El NiñoSouthern Oscillation. Elsevier Oceanography Series, Amsterdam: 55-126.

Glynn, P.W., J. Cortes, H.M. Guzman and R.H. Richmond. 1988. El Niño (1982-83) associated coral mortality and relationship to sea surface temperature deviations in the tropical eastern Pacific. Proc. 6th Int. Coral Reef Symp., Australia, 1988, 3: 237-243.

Green, F. 1983. Comparison of present day coral communities off the Oman coast with mid-Tertiary corals from the MAM reef, near Seeb, Oman. Paper presented to International Society for Reef Studies, December 1983, Nice, France: 6 pp.

Green, F. 1984. Corals of Oman. PDO News, 3/1984: 6-11.

Green, F. and R. Keech. 1986. The coral seas of Muscat. Middle East Economic Digest Ltd., London: 106 pp.

IUCN. 1986. Oman coastal zone management plan. Greater Capital Area. Ministry of Commerce and Industry, Sultanate of Oman: $79 \mathrm{pp}$.

IUCN. 1988. Oman coastal zone management plan. Quriyat to Ra's al Hadd. Ministry of Commerce and Industry, Sultanate of Oman: $57 \mathrm{pp}$. 
IUCN. 1989a. Oman coastal zone management plan. Dhofar. Volume 1. Action Plan. Ministry of Commerce and Industry, Sultanate of Oman: $41 \mathrm{pp}$.

IUCN. 1989b. Oman coastal zone management plan. Dhofar. Volume 2. Resource Atlas. Ministry of Commerce and Industry, Sultanate of Oman: $41 \mathrm{pp}$.

IUCN. 1991. Oman coastal zone management plan. Musandam. Ministry of Commerce and Industry, Sultanate of Oman: $70 \mathrm{pp}$.

Jokiel, P.L. and S.L. Coles. 1990. Response of Hawaiian and other Indo-Pacific reef corals to elevated temperature. Coral Reefs, 8: 155-162.

Rosen, B.R. 1971. The distribution of reef coral genera in the Indian Ocean. Pp. 263299 in D.E. Stoddart and M. Yonge (eds). Regional variation in Indian Ocean coral reefs. Symp. Zool. Soc. Lond., 28. Academic Press, London.

Salm, R.V. 1976. The dynamics and management of the Ponta Torres coral reef, Mozambique. Mems. Inst. Invest. Cient. Mozamb., 12 serie A: 25-40.

Salm, R.V. 1986a. The proposed Daymaniyat Islands National Nature Reserve management plan. Report of IUCN Coastal Zone Management Project Oman: 45 pp.

Salm, R.V. 1986b. Coral reefs and tourist carrying capacity: the Indian Ocean experience. UNEP Industry and Environment, 9(1): 11-14.

Salm, R.V. 1986c. Musandam: overnight trip in Khawr Habalayn (19/4/86-11/4/86). IUCN Coastal Zone Management Project 9224, Sultanate of Oman, CZMP2:F1: 3 pp.

Salm, R.V. 1989. A general overview of the coast between Ra's al Hadd and Dhofar. IUCN Coastal Zone Management Project 9429, Sultanate of Oman, CZMP3:F6: 18 pp.

Salm, R.V. and J. Mee. 1989. Chaetodon dialeucos, sp. nov. A new species of shallow water butterflyfish from the northwest Indian Ocean. F.A.M.A., 12(3): 8-11, 131.

Salm, R.V. and C.R.C. Sheppard. 1986. Corals and coral reefs of the Capital area, Oman. IUCN Coastal Zone Management Project 9070, Sultanate of Oman: 15 pp.

Salm, R.V., Sukotjo and J. Genolagani. 1982. Krakatau: Survey of coastal habitats. Direktorat Perlindungan dan Pengawetan Alam, Bogor, Indonesia. Ms report: 13 pp.

Scheer, G. and C.S.G. Pillai. 1983. Report on the stony corals from the Red Sea. Zoologica, 45: $198 \mathrm{pp}$.

Sheppard, C.R.C. 1986. Marine habitats and species in Oman. Report of IUCN Project 9069, Gland Switzerland: 68 pp., 6 figures. 
Sheppard, C.R.C. and R.V. Salm. 1988. Reef and coral communities of Oman, with description of a new coral species (Order Scleractinia, genus Acanthastrea). J. nat. Hist., 22: 263-279.

Vaughan, T.W. 1914. Reef corals of the Bahamas and of Southern Florida. Carnegie Inst. Wash. Yearbook, 13: 222-226.

Veron, J.E.N. and M. Pichon. 1976. Scleractinia of eastern Australia. Part I. Families Thamnasteriidae, Astrocoeniidae, Pocilloporidae. Australian Institute of Marine Science Monograph Series 1: 86 pp.

Veron, J.E.N. and M. Pichon. 1980. Scleractinia of eastern Australia. Part III. Families Agariciidae, Siderastreidae, Fungiidae, Oculiniidae, Merculinidae, Mussidae, Pectinidae, Caryophylliidae, Dendrophylliidae. Australian Institute of Marine Science Monograph Series 4: $422 \mathrm{pp}$.

Veron, J.E.N. and M. Pichon. 1982. Scleractinia of eastern Australia. Part IV. Family Poritidae. Australian Institute of Marine Science Monograph Series 5: 159 pp.

Veron, J.E.N., M. Pichon and M. Wijsman-Best. 1977. Scleractinia of eastern Australia. Part II. Families Faviidae, Trachyphyllidae. Australian Institute of Marine Science Monograph Series 3: 233 pp.

Veron, J.E.N. and C.C. Wallace. 1984. Scleractinia of eastern Australia. Part V. Family Acroporidae. Australian Institute of Marine Science Monograph Series 6: 495 pp.

Wells, J.W. 1956. Scleractinia. Pp. F328-F440 in R.C. Moore (ed.). Treatise on Invertebrate Paleontology. Part F. Coelenterata. Geol. Soc. Am. and Univ. Kansas Press.

Yonge, C.M. and A.G. Nichols. 1931. Studies on the physiology of corals. IV. The structure, distribution and physiology of the zooxanthellae. Sci. Rep. Gr. Barrier Reef Exped. 1928-219, 1: 135-176. 
APPENDIX 1. CORAL LISTS (coordinates from National Survey Authority Map Series K6611, 1:100,000; for full names of corals see Table 1; Unid = unidentified genus; $G=$ total genera; $S$ = total species; numbers of species listed in columns)

Site Site Name

Map Coordinates Psam Styloc Poc Stylop Ser Mad Ast Acr Monti Pav Leptos Gar Sid

$001 \mathrm{Ra}$ 's al Jirri $\mathrm{N}$

002 Cove 1, RSM

003 Khawr Idah S

004 Shaykh Mas'ud W

005 Ra's Salti Ali

006 Ra's Shakhs

007 Khawr ash Shamm

008 Jazirat Maqlab

009 Maksar

010 Jazirat Sibi

011 Ghassah

012 Khawr Ghubb Ali

013 Khawr Ghubb Ali

014 Khawr Ghubb Ali

015 Umm al Ghanam

016 Umm al Ghanam

017 Umm al Ghanam

$018 \mathrm{Umm}$ al Ghanam

019 Umm al Ghanam

020 Khawr al Quway'

021 Ra's Rarak

022 Khawr Khayran

023 Khawr Ran

024 Jazirat Abu Sir

025 Jazirat Abu Sir

026 Khawr Kumzar

027 "Salla"

'028 Khawr Ma'ili

029 Jazirat al Khayl S

030 Ghubbat al Khouse

031 Ghubbat al Khouse

032 Ghubbat al Khouse

033 Ghubbat al Khouse

034 Ghubbat al Khouse

035 Jazirat Bu Rashid

036 Jazirat Musandam

037 Jazirat Musandam

038 Jazirat Musandam

039 Jazirat Musandam

040 Dawhat ash Shisah

041 Dawhat ash Shisah

042 Dawhat ash Shisah

043 Dawhat ash Shisah

044 Dawhat ash Shisah

045 Dawhat ash Shisah

046 Khaysah-Bashin

047 Ra's Khaysah S

048 Ra's Bashin S

049 Umm al Fayyarin

050 Ghubbat Shabus

051 Ghubbat Shabus
DQ198022

DQ200026

DQ214041

DP237994

DP280997-284998

DP340980

DP344974

DP395980

DQ394004

DQ320026

DQ350060

DQ324075

DQ368060

DQ344126

DQ356167

DQ363170-366167

DQ361163-360152

DQ358132-352127

DQ374169

DQ386174

DQ390160

DQ410164-414169

DQ420182

DQ418174

DQ417156-416148

DQ432147-440146

DQ442146-440132

DQ449165-462160

DQ480130

DQ462126-460115

DQ500118

DQ490137

DQ478145

DQ495204

DQ525176

DQ526184

DQ535165

DQ517156

DQ440060

DQ404080

DQ410055

$\mathrm{DQ} 411028$

$\mathrm{DQ} 425016$

DQ462018

DQ494017-487968

DQ476004

DQ485968

DP548955

DQ460960

DP440957

\section{Psam Styloc Poc Stylop Ser Mad Ast Acr Monti Pav Leplos Gar Sid}

$\begin{array}{lllllllllllll}0 & 0 & 1 & 1 & 0 & 0 & 0 & 3 & 2 & 0 & 0 & 0 & 0\end{array}$

$\begin{array}{lllllllllllll}0 & 0 & 0 & 0 & 0 & 0 & 0 & 2 & 1 & 0 & 0 & 0 & 0\end{array}$

$\begin{array}{lllllllllllll}0 & 0 & 1 & 1 & 0 & 0 & 0 & 3 & 2 & 0 & 0 & 0 & 0\end{array}$

$\begin{array}{lllllllllllll}0 & 0 & 1 & 0 & 0 & 0 & 0 & 2 & 1 & 0 & 0 & 0 & 0\end{array}$

$\begin{array}{lllllllllllll}1 & 0 & 1 & 1 & 0 & 0 & 0 & 3 & 1 & 0 & 0 & 0 & 0\end{array}$

$\begin{array}{lllllllllllll}1 & 0 & 1 & 0 & 0 & 0 & 0 & 4 & 1 & 0 & 0 & 0 & 0\end{array}$

$\begin{array}{lllllllllllll}1 & 0 & 1 & 1 & 0 & 1 & 0 & 4 & 2 & 4 & 0 & 0 & 1\end{array}$

$\begin{array}{lllllllllllll}1 & 0 & 1 & 1 & 0 & 1 & 0 & 4 & 2 & 3 & 0 & 0 & 0\end{array}$

$\begin{array}{lllllllllllll}1 & 0 & 0 & 1 & 0 & 0 & 0 & 3 & 0 & 4 & 0 & 0 & 1\end{array}$

$\begin{array}{lllllllllllll}1 & 0 & 0 & 1 & 0 & 0 & 0 & 3 & 0 & 2 & 0 & 0 & 0\end{array}$

$\begin{array}{lllllllllllll}1 & 0 & 1 & 1 & 0 & 0 & 0 & 4 & 1 & 1 & 0 & 0 & 0\end{array}$

$\begin{array}{lllllllllllll}1 & 0 & 1 & 1 & 0 & 1 & 0 & 4 & 1 & 1 & 0 & 0 & 1\end{array}$

$\begin{array}{lllllllllllll}1 & 0 & 1 & 1 & 0 & 0 & 0 & 4 & 1 & 1 & 0 & 0 & 1\end{array}$

$\begin{array}{lllllllllllll}1 & 0 & 1 & 0 & 0 & 0 & 0 & 3 & 1 & 1 & 0 & 0 & 0\end{array}$

$\begin{array}{lllllllllllll}1 & 0 & 1 & 1 & 0 & 0 & 0 & 3 & 0 & 2 & 0 & 0 & 1\end{array}$

$\begin{array}{lllllllllllll}0 & 0 & 1 & 1 & 0 & 0 & 0 & 2 & 2 & 1 & 0 & 0 & 0\end{array}$

$\begin{array}{llllllllllll}0 & 1 & 1 & 0 & 0 & 1 & 4 & 2 & 2 & 0 & 0 & 1\end{array}$

$\begin{array}{llllllllllll}0 & 1 & 1 & 0 & 0 & 0 & 3 & 2 & 1 & 0 & 0 & 0\end{array}$

$\begin{array}{llllllllllll}0 & 1 & 1 & 0 & 0 & 0 & 3 & 2 & 1 & 0 & 0 & 0\end{array}$

$\begin{array}{llllllllllll}0 & 1 & 1 & 0 & 0 & 0 & 2 & 1 & 1 & 0 & 0 & 0\end{array}$

$\begin{array}{llllllllllll}0 & 1 & 1 & 0 & 0 & 0 & 2 & 1 & 2 & 0 & 0 & 0\end{array}$

$\begin{array}{llllllllllll}0 & 1 & 1 & 0 & 0 & 1 & 4 & 2 & 1 & 0 & 0 & 0\end{array}$

$\begin{array}{llllllllllll}0 & 1 & 1 & 0 & 0 & 1 & 4 & 1 & 1 & 0 & 0 & 0\end{array}$

$\begin{array}{llllllllllll}0 & 1 & 1 & 0 & 0 & 0 & 4 & 1 & 2 & 0 & 0 & 0\end{array}$

$\begin{array}{llllllllllll}0 & 1 & 0 & 0 & 0 & 0 & 3 & 1 & 1 & 0 & 0 & 0\end{array}$

$\begin{array}{llllllllllll}0 & 1 & 1 & 0 & 0 & 1 & 2 & 1 & 1 & 0 & 0 & 0\end{array}$

$\begin{array}{llllllllllll}0 & 1 & 1 & 0 & 0 & 1 & 2 & 2 & 1 & 0 & 0 & 0\end{array}$

$\begin{array}{llllllllllll}0 & 1 & 1 & 0 & 0 & 1 & 2 & 2 & 1 & 0 & 0 & 0\end{array}$

$\begin{array}{llllllllllll}0 & 1 & 0 & 0 & 0 & 0 & 2 & 2 & 2 & 0 & 0 & 0\end{array}$

$\begin{array}{llllllllllll}0 & 1 & 1 & 0 & 0 & 1 & 4 & 3 & 2 & 0 & 0 & 1\end{array}$

$\begin{array}{llllllllllll}0 & 1 & 1 & 0 & 0 & 1 & 3 & 1 & 2 & 0 & 0 & 0\end{array}$

$\begin{array}{llllllllllll}0 & 1 & 0 & 0 & 0 & 0 & 1 & 0 & 1 & 0 & 0 & 0\end{array}$

$\begin{array}{llllllllllll}0 & 1 & 1 & 0 & 0 & 0 & 4 & 3 & 2 & 0 & 0 & 1\end{array}$

$\begin{array}{llllllllllll}0 & 1 & 1 & 0 & 0 & 0 & 3 & 1 & 2 & 0 & 0 & 1\end{array}$

$\begin{array}{llllllllllll}0 & 1 & 1 & 0 & 0 & 0 & 3 & 2 & 1 & 0 & 0 & 0\end{array}$

$\begin{array}{llllllllllll}0 & 1 & 1 & 0 & 0 & 0 & 2 & 3 & 1 & 0 & 0 & 0\end{array}$

$\begin{array}{llllllllllll}0 & 1 & 1 & 0 & 0 & 0 & 2 & 1 & 0 & 0 & 0 & 0\end{array}$

$\begin{array}{llllllllllll}0 & 1 & 1 & 0 & 0 & 0 & 2 & 2 & 1 & 0 & 0 & 0\end{array}$

$\begin{array}{llllllllllll}0 & 1 & 1 & 0 & 0 & 0 & 4 & 2 & 1 & 0 & 0 & 1\end{array}$

$\begin{array}{llllllllllll}1 & 1 & 1 & 0 & 0 & 1 & 4 & 2 & 2 & 0 & 0 & 1\end{array}$

$\begin{array}{llllllllllll}0 & 1 & 0 & 0 & 0 & 1 & 2 & 2 & 1 & 0 & 0 & 0\end{array}$

$\begin{array}{llllllllllll}0 & 1 & 1 & 0 & 0 & 1 & 2 & 1 & 1 & 0 & 0 & 0\end{array}$

$\begin{array}{llllllllllll}0 & 1 & 1 & 0 & 0 & 0 & 3 & 0 & 1 & 0 & 0 & 0\end{array}$

$\begin{array}{llllllllllll}1 & 1 & 0 & 0 & 0 & 1 & 4 & 1 & 2 & 0 & 0 & 1\end{array}$

$\begin{array}{llllllllllll}0 & 1 & 0 & 0 & 0 & 1 & 3 & 0 & 1 & 0 & 0 & 1\end{array}$

$\begin{array}{llllllllllll}0 & 1 & 0 & 0 & 0 & 0 & 3 & 2 & 1 & 0 & 0 & 0\end{array}$

$\begin{array}{llllllllllll}0 & 1 & 0 & 0 & 0 & 1 & 2 & 1 & 0 & 0 & 0 & 0\end{array}$

$\begin{array}{llllllllllll}0 & 1 & 1 & 0 & 0 & 0 & 4 & 2 & 2 & 0 & 0 & 1\end{array}$

$\begin{array}{lllllllllllll}0 & 0 & 1 & 1 & 0 & 0 & 0 & 2 & 1 & 1 & 0 & 0 & 0 \\ 1 & 0 & 1 & 1 & 0 & 0 & 1 & 3 & 2 & 2 & 0 & 0 & 1\end{array}$ 
APPENDIX 1. Continued

Site Pseud Ano Cos Por P(Syn) Gonio Alv Favia Favit Gonia Pla Leptor Monta Ples Lepta Cyph Echin Cul Phy Gal Par Hyd

$\begin{array}{llllllllllllllllllllllll}001 & 0 & 1 & 0 & 1 & 0 & 1 & 0 & 0 & 2 & 0 & 1 & 0 & 0 & 0 & 1 & 1 & 0 & 0 & 0 & 0 & 0 & 0\end{array}$ $\begin{array}{lllllllllllllllllllllll}002 & 0 & 1 & 0 & 1 & 0 & 1 & 0 & 0 & 1 & 0 & 1 & 0 & 0 & 0 & 0 & 1 & 0 & 0 & 0 & 0 & 0 & 0\end{array}$ $\begin{array}{lllllllllllllllllllllll}003 & 0 & 1 & 0 & 1 & 0 & 1 & 0 & 0 & 2 & 0 & 1 & 0 & 0 & 0 & 1 & 1 & 0 & 0 & 0 & 0 & 0 & 0\end{array}$ $\begin{array}{llllllllllllllllllllllll}004 & 0 & 1 & 0 & 1 & 0 & 1 & 0 & 0 & 1 & 0 & 1 & 0 & 0 & 0 & 1 & 1 & 0 & 0 & 0 & 0 & 0 & 0\end{array}$ $\begin{array}{lllllllllllllllllllllll}005 & 0 & 0 & 0 & 2 & 0 & 1 & 0 & 2 & 0 & 0 & 2 & 0 & 0 & 0 & 1 & 2 & 0 & 0 & 0 & 0 & 0 & 1\end{array}$ $\begin{array}{lllllllllllllllllllllll}006 & 0 & 0 & 0 & 1 & 0 & 0 & 0 & 2 & 0 & 0 & 1 & 0 & 0 & 0 & 2 & 1 & 0 & 0 & 0 & 0 & 0 & 0\end{array}$ $\begin{array}{llllllllllllllllllllllll}007 & 1 & 1 & 1 & 2 & 0 & 1 & 0 & 4 & 1 & 0 & 2 & 0 & 0 & 1 & 2 & 1 & 0 & 0 & 0 & 0 & 0 & 0\end{array}$ $\begin{array}{llllllllllllllllllllllll}008 & 1 & 1 & 1 & 2 & 0 & 1 & 0 & 3 & 1 & 0 & 2 & 0 & 0 & 1 & 2 & 1 & 0 & 0 & 0 & 0 & 0 & 0\end{array}$ $\begin{array}{lllllllllllllllllllllll}009 & 0 & 0 & 1 & 1 & 0 & 0 & 0 & 4 & 1 & 0 & 2 & 0 & 0 & 1 & 2 & 1 & 0 & 0 & 0 & 0 & 0 & 0\end{array}$ $\begin{array}{lllllllllllllllllllllll}010 & 0 & 0 & 1 & 1 & 0 & 0 & 0 & 4 & 1 & 0 & 2 & 0 & 0 & 0 & 1 & 1 & 0 & 0 & 0 & 0 & 0 & 0\end{array}$ $\begin{array}{lllllllllllllllllllllll}011 & 0 & 0 & 0 & 2 & 0 & 1 & 0 & 0 & 1 & 0 & 1 & 0 & 0 & 0 & 1 & 0 & 0 & 0 & 0 & 0 & 0 & 0\end{array}$ $\begin{array}{lllllllllllllllllllllll}012 & 0 & 1 & 1 & 3 & 0 & 1 & 0 & 2 & 2 & 0 & 2 & 0 & 0 & 1 & 2 & 1 & 1 & 0 & 0 & 0 & 0 & 1\end{array}$ $\begin{array}{lllllllllllllllllllllll}013 & 0 & 1 & 1 & 3 & 0 & 1 & 0 & 2 & 2 & 0 & 2 & 0 & 0 & 1 & 2 & 1 & 1 & 0 & 0 & 0 & 0 & 1\end{array}$ $\begin{array}{lllllllllllllllllllllll}014 & 0 & 0 & 1 & 1 & 0 & 0 & 0 & 2 & 1 & 0 & 1 & 0 & 0 & 0 & 1 & 1 & 0 & 0 & 0 & 0 & 0 & 0\end{array}$ $\begin{array}{lllllllllllllllllllllll}015 & 1 & 1 & 0 & 1 & 0 & 1 & 0 & 2 & 2 & 0 & 1 & 0 & 0 & 1 & 2 & 2 & 1 & 0 & 0 & 0 & 0 & 2\end{array}$ $\begin{array}{lllllllllllllllllllllll}016 & 0 & 1 & 1 & 2 & 0 & 1 & 0 & 3 & 1 & 0 & 2 & 0 & 0 & 0 & 2 & 1 & 0 & 0 & 0 & 0 & 0 & 2\end{array}$ $\begin{array}{lllllllllllllllllllllll}017 & 1 & 1 & 1 & 2 & 0 & 2 & 0 & 3 & 2 & 0 & 2 & 0 & 0 & 0 & 2 & 2 & 1 & 0 & 0 & 0 & 0 & 2\end{array}$ $\begin{array}{lllllllllllllllllllllll}018 & 1 & 1 & 1 & 2 & 0 & 1 & 0 & 0 & 2 & 0 & 2 & 0 & 0 & 0 & 1 & 0 & 1 & 0 & 0 & 0 & 0 & 1\end{array}$ $\begin{array}{lllllllllllllllllllllll}019 & 0 & 1 & 0 & 3 & 0 & 1 & 0 & 2 & 3 & 0 & 2 & 0 & 0 & 0 & 1 & 1 & 1 & 0 & 0 & 0 & 0 & 0\end{array}$ $\begin{array}{lllllllllllllllllllllll}020 & 1 & 1 & 1 & 2 & 0 & 1 & 0 & 3 & 1 & 0 & 2 & 0 & 0 & 0 & 1 & 1 & 0 & 0 & 0 & 0 & 0 & 1\end{array}$ $\begin{array}{lllllllllllllllllllllll}021 & 0 & 1 & 1 & 1 & 0 & 1 & 0 & 2 & 2 & 0 & 1 & 0 & 0 & 1 & 2 & 1 & 1 & 0 & 0 & 0 & 0 & 2\end{array}$ $\begin{array}{lllllllllllllllllllllll}022 & 1 & 1 & 1 & 3 & 0 & 3 & 0 & 3 & 1 & 0 & 2 & 0 & 0 & 0 & 2 & 2 & 1 & 0 & 0 & 0 & 0 & 2\end{array}$ $\begin{array}{lllllllllllllllllllllll}023 & 1 & 1 & 1 & 1 & 0 & 2 & 0 & 2 & 2 & 0 & 1 & 0 & 0 & 1 & 3 & 2 & 1 & 0 & 0 & 0 & 0 & 2\end{array}$ $\begin{array}{lllllllllllllllllllllll}024 & 1 & 1 & 1 & 3 & 0 & 1 & 0 & 2 & 2 & 0 & 2 & 0 & 0 & 0 & 0 & 1 & 1 & 0 & 0 & 0 & 0 & 2\end{array}$ $\begin{array}{lllllllllllllllllllllll}025 & 0 & 0 & 0 & 1 & 0 & 0 & 0 & 1 & 2 & 0 & 1 & 0 & 0 & 0 & 2 & 1 & 1 & 0 & 0 & 0 & 0 & 2\end{array}$ $\begin{array}{lllllllllllllllllllllll}026 & 0 & 1 & 1 & 1 & 0 & 2 & 0 & 2 & 1 & 0 & 2 & 0 & 0 & 0 & 1 & 1 & 1 & 0 & 0 & 0 & 0 & 1\end{array}$ $\begin{array}{lllllllllllllllllllllll}027 & 1 & 1 & 1 & 1 & 0 & 1 & 0 & 1 & 2 & 0 & 2 & 0 & 0 & 0 & 2 & 1 & 1 & 0 & 0 & 0 & 0 & 2\end{array}$ $\begin{array}{lllllllllllllllllllllll}028 & 0 & 1 & 1 & 1 & 0 & 1 & 0 & 1 & 2 & 0 & 2 & 0 & 0 & 0 & 2 & 2 & 1 & 0 & 0 & 0 & 0 & 0\end{array}$ $\begin{array}{lllllllllllllllllllllll}029 & 1 & 0 & 1 & 1 & 1 & 2 & 0 & 1 & 2 & 0 & 1 & 0 & 0 & 1 & 1 & 1 & 1 & 0 & 0 & 0 & 0 & 2\end{array}$ $\begin{array}{lllllllllllllllllllllll}030 & 1 & 1 & 1 & 1 & 0 & 2 & 0 & 2 & 1 & 0 & 1 & 0 & 0 & 1 & 3 & 1 & 1 & 0 & 0 & 0 & 1 & 2\end{array}$ $\begin{array}{lllllllllllllllllllllll}031 & 0 & 0 & 1 & 1 & 0 & 2 & 0 & 0 & 1 & 0 & 1 & 0 & 0 & 0 & 1 & 1 & 1 & 0 & 0 & 0 & 1 & 1\end{array}$ $\begin{array}{lllllllllllllllllllllll}032 & 0 & 0 & 0 & 1 & 0 & 1 & 0 & 1 & 1 & 0 & 0 & 0 & 0 & 1 & 1 & 1 & 0 & 0 & 0 & 0 & 0 & 0\end{array}$ $\begin{array}{lllllllllllllllllllllll}033 & 1 & 1 & 1 & 2 & 0 & 2 & 0 & 2 & 1 & 0 & 1 & 0 & 0 & 1 & 3 & 1 & 1 & 0 & 0 & 0 & 1 & 2\end{array}$ $\begin{array}{lllllllllllllllllllllll}034 & 1 & 0 & 1 & 2 & 0 & 1 & 0 & 1 & 1 & 0 & 2 & 0 & 0 & 0 & 2 & 2 & 1 & 0 & 0 & 0 & 0 & 1\end{array}$ $\begin{array}{lllllllllllllllllllllll}035 & 1 & 1 & 1 & 1 & 0 & 2 & 0 & 3 & 3 & 0 & 1 & 0 & 0 & 1 & 3 & 0 & 1 & 0 & 0 & 0 & 0 & 2\end{array}$ $\begin{array}{lllllllllllllllllllllll}036 & 0 & 0 & 0 & 1 & 0 & 1 & 0 & 1 & 1 & 0 & 1 & 0 & 0 & 1 & 1 & 1 & 1 & 0 & 0 & 0 & 0 & 2\end{array}$ $\begin{array}{lllllllllllllllllllllll}037 & 0 & 0 & 0 & 1 & 0 & 1 & 0 & 1 & 2 & 0 & 1 & 0 & 0 & 0 & 0 & 1 & 0 & 0 & 0 & 0 & 0 & 1\end{array}$ $\begin{array}{lllllllllllllllllllllll}038 & 0 & 0 & 0 & 2 & 0 & 1 & 0 & 1 & 2 & 0 & 1 & 0 & 0 & 0 & 0 & 0 & 1 & 0 & 0 & 0 & 0 & 1\end{array}$ $\begin{array}{lllllllllllllllllllllll}039 & 0 & 0 & 1 & 1 & 0 & 1 & 0 & 1 & 2 & 0 & 1 & 0 & 0 & 0 & 1 & 1 & 1 & 0 & 0 & 0 & 0 & 2\end{array}$ $\begin{array}{lllllllllllllllllllllll}040 & 1 & 1 & 1 & 2 & 0 & 2 & 0 & 2 & 2 & 0 & 3 & 0 & 0 & 1 & 3 & 2 & 1 & 0 & 0 & 0 & 1 & 2\end{array}$ $\begin{array}{lllllllllllllllllllllll}041 & 0 & 1 & 1 & 1 & 0 & 1 & 0 & 1 & 2 & 0 & 2 & 0 & 0 & 0 & 1 & 1 & 1 & 0 & 0 & 0 & 0 & 1\end{array}$ $\begin{array}{lllllllllllllllllllllll}042 & 0 & 0 & 1 & 1 & 0 & 2 & 0 & 1 & 2 & 0 & 1 & 0 & 0 & 0 & 2 & 1 & 1 & 0 & 0 & 0 & 1 & 2\end{array}$ $\begin{array}{lllllllllllllllllllllll}043 & 0 & 0 & 0 & 2 & 0 & 1 & 0 & 0 & 1 & 0 & 2 & 0 & 0 & 0 & 2 & 0 & 1 & 0 & 0 & 0 & 1 & 1\end{array}$ $\begin{array}{lllllllllllllllllllllll}044 & 1 & 1 & 1 & 2 & 1 & 2 & 0 & 2 & 3 & 0 & 3 & 0 & 0 & 1 & 3 & 2 & 1 & 0 & 0 & 0 & 0 & 2\end{array}$ $\begin{array}{lllllllllllllllllllllll}045 & 0 & 0 & 1 & 1 & 0 & 1 & 0 & 1 & 3 & 0 & 2 & 0 & 0 & 1 & 0 & 0 & 1 & 0 & 0 & 0 & 0 & 2\end{array}$ $\begin{array}{lllllllllllllllllllllll}046 & 0 & 1 & 0 & 1 & 0 & 2 & 0 & 1 & 1 & 0 & 2 & 0 & 0 & 0 & 3 & 1 & 1 & 0 & 0 & 0 & 0 & 2\end{array}$ $\begin{array}{lllllllllllllllllllllll}047 & 0 & 0 & 1 & 2 & 0 & 1 & 0 & 1 & 2 & 0 & 1 & 0 & 0 & 0 & 2 & 1 & 1 & 0 & 0 & 0 & 1 & 2\end{array}$ $\begin{array}{lllllllllllllllllllllll}048 & 0 & 1 & 1 & 2 & 0 & 1 & 0 & 1 & 3 & 0 & 2 & 0 & 0 & 0 & 2 & 1 & 1 & 0 & 0 & 0 & 1 & 2\end{array}$ $\begin{array}{lllllllllllllllllllllll}049 & 1 & 0 & 1 & 1 & 0 & 1 & 0 & 1 & 1 & 0 & 1 & 0 & 0 & 1 & 1 & 0 & 1 & 0 & 0 & 0 & 0 & 0\end{array}$ $\begin{array}{lllllllllllllllllllllll}050 & 1 & 1 & 1 & 2 & 0 & 2 & 0 & 1 & 3 & 0 & 2 & 0 & 0 & 1 & 2 & 1 & 2 & 0 & 0 & 0 & 1 & 2\end{array}$ $\begin{array}{lllllllllllllllllllllll}051 & 0 & 1 & 1 & 1 & 0 & 1 & 0 & 1 & 2 & 0 & 2 & 0 & 0 & 1 & 2 & 1 & 2 & 0 & 0 & 0 & 1 & 1\end{array}$ 
APPENDIX 1. Continued

Site Bla Sym Aca Echinoph Oxy Euph Parac Poly Heteroc Cary Bal Rhiz Den Tub Tur Heterop Tubip Mille Stylas Unid G S

\begin{tabular}{|c|c|c|c|c|c|c|c|c|c|c|c|c|c|c|c|c|c|c|c|c|c|c|}
\hline 001 & 0 & 0 & 1 & 0 & 0 & 0 & 0 & 0 & 0 & 0 & 0 & 0 & 0 & 0 & 0 & 0 & 0 & 0 & 0 & 0 & 12 & 16 \\
\hline 002 & 0 & 0 & 1 & 0 & 0 & 0 & 0 & 0 & 0 & 0 & 0 & 0 & 0 & 0 & 0 & 0 & 0 & 0 & 0 & 0 & 9 & 10 \\
\hline 003 & 0 & 0 & 1 & 0 & 0 & 0 & 0 & 0 & 0 & 0 & 0 & 0 & 0 & 0 & 0 & 0 & 0 & 0 & 0 & 0 & 12 & 16 \\
\hline 004 & 0 & 0 & 1 & 0 & 0 & 0 & 0 & 0 & 0 & 0 & 0 & 0 & 0 & 0 & 0 & 0 & 0 & 0 & 0 & 0 & 11 & 12 \\
\hline 005 & 0 & 1 & 1 & 0 & 0 & 0 & 0 & 0 & 0 & 0 & 0 & 0 & 0 & 0 & 0 & 0 & 0 & 0 & 0 & 0 & 14 & 20 \\
\hline 006 & 0 & 0 & 0 & 0 & 0 & 0 & 0 & 0 & & ( & 0 & 0 & 0 & 0 & 0 & 0 & 0 & 0 & 0 & 0 & 9 & 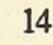 \\
\hline 007 & 0 & 0 & 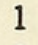 & & 1 & 0 & 0 & 0 & & & 0 & 0 & 0 & 0 & 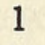 & 0 & & 0 & & 0 & 23 & 36 \\
\hline 008 & 0 & 0 & & & 0 & 0 & 0 & 0 & & & 0 & 0 & 0 & 0 & 1 & ( & & 0 & & & 21 & 32 \\
\hline 009 & 0 & 0 & 0 & & 1 & 0 & 0 & 0 & & & 0 & 0 & 0 & 0 & 0 & 0 & & 0 & & , & 15 & 25 \\
\hline 010 & 0 & 0 & 0 & 1 & 0 & 0 & 0 & 0 & 0 & C & 0 & 0 & 0 & 0 & 0 & 0 & & 0 & & 0 & 12 & 19 \\
\hline 011 & 0 & 0 & 1 & 0 & 0 & 0 & 0 & 0 & 0 & C & 0 & 0 & 0 & 0 & 0 & 0 & 0 & 0 & 0 & 0 & 12 & 16 \\
\hline 012 & 0 & 1 & . & 1 & 0 & 0 & 1 & 0 & $c$ & C & 0 & 0 & 1 & 1 & 0 & 0 & & 0 & & 0 & 26 & 35 \\
\hline 013 & 0 & 1 & 1 & & 0 & 0 & 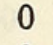 & 0 & & & 0 & 0 & 0 & 1 & 0 & 0 & & & & 0 & 23 & 32 \\
\hline 014 & 0 & 0 & 0 & 0 & 0 & 0 & 0 & 0 & & & 0 & 0 & 0 & 0 & 0 & 0 & & & & 0 & 12 & 15 \\
\hline 015 & 0 & 0 & 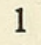 & 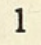 & 0 & 0 & 0 & 0 & & ( & 0 & 0 & 0 & 0 & 0 & 0 & & 0 & & 0 & 20 & 28 \\
\hline 016 & 0 & 1 & 1 & 1 & 0 & 0 & 0 & 0 & c & $c$ & 0 & 0 & 0 & 0 & 0 & 0 & & 0 & & 0 & 18 & 26 \\
\hline 017 & 0 & 1 & 1 & 1 & 1 & 0 & 0 & 0 & 0 & 0 & 0 & 0 & 0 & 1 & 1 & 0 & 0 & 0 & 0 & 0 & 26 & 40 \\
\hline 018 & 0 & 1 & 0 & 1 & 0 & 0 & 0 & 0 & 0 & c & 0 & 0 & 0 & 1 & 0 & 0 & & 0 & 0 & 0 & 19 & 25 \\
\hline 019 & 0 & 0 & 1 & 0 & 0 & 0 & 0 & 0 & 0 & $\mathrm{C}$ & 0 & 0 & 0 & 1 & 0 & 8 & & & & 0 & 16 & 25 \\
\hline 020 & 0 & 1 & 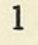 & 0 & 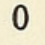 & 0 & 0 & 0 & & 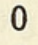 & 0 & 0 & 0 & 0 & 0 & 0 & & & & 0 & 19 & 24 \\
\hline 021 & 1 & 1 & 1 & 1 & 1 & 0 & 0 & 0 & & & 0 & 0 & 0 & 0 & 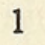 & 0 & & & & 0 & 24 & 30 \\
\hline 022 & 0 & 1 & 1 & 1 & 1 & 0 & 0 & 0 & 0 & 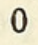 & 0 & 0 & 0 & 0 & 1 & 0 & & 0 & & 0 & 23 & 37 \\
\hline 023 & 0 & 1 & 1 & 1 & 1 & 0 & 0 & 0 & 0 & 0 & 0 & 0 & 0 & 1 & 0 & 0 & & 0 & 0 & 0 & 25 & 35 \\
\hline 024 & 0 & 1 & 1 & 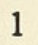 & 1 & 0 & 0 & 0 & 0 & 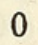 & 0 & 0 & 0 & 1 & 0 & 0 & 0 & 0 & 0 & 0 & 22 & 32 \\
\hline 025 & 0 & 1 & 1 & 1 & 0 & 0 & 0 & 0 & 0 & 0 & 0 & 0 & 0 & 0 & 1 & 0 & 0 & ( & 0 & 0 & 16 & 21 \\
\hline 026 & 0 & 1 & 1 & 1 & 0 & 0 & 0 & 0 & 0 & 0 & 0 & 0 & 0 & 0 & 0 & 0 & & & & 0 & 20 & 24 \\
\hline 027 & 0 & 1 & 1 & 1 & 0 & 0 & 0 & 0 & & C & 0 & 0 & 0 & 0 & 1 & 0 & & & & 0 & 23 & 29 \\
\hline 028 & 0 & 1 & 1 & 1 & 1 & 0 & 0 & 0 & & C & 0 & 0 & 0 & 0 & 0 & 0 & & & & 0 & 21 & 28 \\
\hline 029 & 0 & 1 & 0 & 1 & 1 & 0 & 0 & 0 & 0 & 0 & 0 & 0 & 0 & 0 & 0 & $v$ & & & & 0 & 21 & 27 \\
\hline 030 & 1 & 1 & 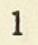 & 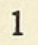 & 1 & 0 & 0 & 0 & 0 & 0 & 0 & 0 & 0 & 1 & 1 & 0 & 0 & 0 & 0 & 0 & 29 & 40 \\
\hline 031 & 0 & 1 & 1 & 1 & 1 & 0 & 0 & 0 & 0 & 0 & 0 & 0 & 0 & 0 & 0 & 0 & 0 & 0 & 0 & 0 & 21 & 25 \\
\hline 032 & 0 & 1 & 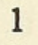 & 1 & 0 & 0 & 0 & 0 & 0 & 0 & 0 & 0 & 0 & 0 & 0 & 0 & 0 & 0 & 0 & 0 & 14 & 14 \\
\hline 033 & 1 & 1 & . & 1 & 1 & 0 & 0 & 0 & ( & 0 & 0 & 0 & 0 & 1 & 1 & 0 & 0 & ( & & 0 & 27 & 39 \\
\hline 034 & 0 & 1 & 1 & 1 & 1 & 0 & 0 & 0 & 0 & 0 & 0 & 0 & 0 & 0 & 0 & 0 & & & & 0 & 22 & 29 \\
\hline 035 & 0 & 1 & 1 & 1 & 0 & 0 & 0 & 0 & 0 & 0 & 0 & 1 & 1 & 1 & 0 & 0 & & & 0 & 0 & 24 & 35 \\
\hline 036 & 0 & 1 & 1 & 1 & 0 & 0 & 0 & 0 & 0 & 0 & 0 & 0 & 0 & 0 & 0 & 0 & 0 & C & 0 & 0 & 19 & 23 \\
\hline 037 & 0 & 1 & 1 & 0 & 0 & 0 & 0 & 0 & 0 & 0 & 0 & 0 & 0 & 0 & 0 & 0 & 0 & $c$ & 0 & 0 & 13 & 15 \\
\hline 038 & 0 & 1 & 0 & 1 & 1 & 0 & 0 & 0 & 0 & 0 & 0 & 0 & 0 & 0 & 0 & 0 & 0 & 0 & 0 & 0 & 15 & 19 \\
\hline 039 & 0 & 1 & 1 & ( & 0 & 0 & 0 & 0 & 0 & 0 & 0 & 0 & 0 & 1 & 0 & 0 & 0 & 0 & 0 & 0 & 20 & 26 \\
\hline 040 & 1 & 1 & 1 & 1 & 1 & 0 & 0 & 0 & ( & ( & 0 & 0 & 0 & 1 & 1 & 0 & 0 & 0 & 0 & 0 & 30 & 45 \\
\hline 041 & 0 & 1 & 1 & 1 & 0 & 0 & 0 & 0 & 0 & 0 & 0 & 0 & 0 & 0 & 0 & 0 & 0 & & 0 & 0 & 20 & 24 \\
\hline 042 & 1 & 1 & 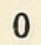 & 1 & 1 & 0 & 0 & 0 & 0 & 0 & 0 & 0 & 0 & 0 & 1 & 0 & 0 & 0 & 0 & 0 & 22 & 27 \\
\hline 043 & 0 & 1 & 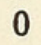 & 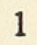 & 1 & 0 & 0 & 0 & 0 & 0 & 0 & 0 & 0 & 0 & 1 & 0 & 0 & 0 & 0 & 0 & 16 & 21 \\
\hline 044 & 1 & 1 & 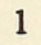 & 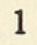 & 1 & 0 & 0 & 0 & 0 & 0 & 0 & 0 & 0 & 0 & 1 & 0 & 0 & 0 & 0 & 0 & 27 & 42 \\
\hline 045 & 1 & 1 & 1 & 1 & 1 & 0 & 0 & 0 & 0 & 0 & 0 & 0 & 0 & 1 & 0 & 0 & 0 & 0 & 0 & 0 & 20 & 26 \\
\hline 046 & 0 & 1 & 1 & 1 & 1 & 0 & 0 & 0 & 0 & 0 & 0 & 0 & 0 & 0 & 0 & 0 & 0 & 0 & 0 & 0 & 19 & 27 \\
\hline 047 & 0 & 1 & 1 & 1 & 2 & 0 & 0 & 0 & 0 & 0 & 0 & 0 & 0 & 0 & 0 & 0 & 0 & 0 & 0 & 0 & 20 & 26 \\
\hline 048 & 1 & 1 & 1 & 1 & 1 & 0 & 0 & 0 & 0 & 0 & 0 & 0 & 0 & 0 & 1 & 0 & 0 & 0 & 0 & 0 & 24 & 35 \\
\hline 049 & 0 & 1 & 1 & $c$ & 0 & 0 & 0 & 0 & 0 & & 0 & 0 & 1 & 1 & 1 & 0 & 0 & ( & 0 & ) & 20 & 21 \\
\hline 050 & 1 & 1 & 1 & 1 & 1 & 0 & 0 & 0 & 0 & 0 & 0 & 0 & 1 & 1 & 1 & 0 & 0 & $c$ & 0 & 0 & 30 & 42 \\
\hline 051 & 1 & 1 & 1 & 1 & 1 & 0 & 0 & 0 & 0 & 0 & 0 & 0 & 1 & 1 & 1 & 0 & 0 & 0 & 0 & 0 & 29 & \\
\hline
\end{tabular}


APPENDIX 1. Continued

$\underline{\text { Site Site Name }}$

Map Coordinates Psam Styloc Poc Stylop Ser Mad Ast Acr Monti Pav Leptos Gar Sid

\begin{tabular}{|c|c|c|c|c|c|c|c|c|c|c|c|c|c|}
\hline 052 Ghubbat Shabus & DP475925-480916 & 1 & 0 & 1 & 1 & 0 & 0 & 1 & 3 & 2 & 1 & 0 & 0 \\
\hline 053 Ra's Dillah N & DP486909 & 0 & 0 & 1 & 0 & 0 & 0 & 0 & 2 & 1 & 0 & 0 & 0 \\
\hline 054 Khawr Habalayn & DP400900 & 2 & 1 & 1 & 1 & 0 & 0 & 1 & 5 & 2 & 2 & 0 & 0 \\
\hline 055 Khawr Habalayn & DP474906-473911 & 1 & 0 & 1 & 0 & 0 & 0 & 1 & 4 & 2 & 1 & 0 & 0 \\
\hline 056 Khawr Habalayn & DP425911 & 1 & 0 & 1 & 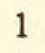 & 0 & 0 & 1 & 4 & 2 & 1 & 0 & 0 \\
\hline 057 Khawr Habalayn & DP414950 & 0 & c & 1 & r. & 0 & 0 & 0 & 4 & 1 & 1 & 0 & 0 \\
\hline 058 Khawr Habalayn & DP395977 & 1 & c & 0 & 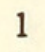 & 0 & 0 & 0 & 3 & 1 & 3 & 0 & 0 \\
\hline 059 Khawr Habalayn & DP357938 & 0 & ( & 1 & . & 0 & 0 & 1 & 5 & 1 & 2 & 0 & 0 \\
\hline 060 Khawr Najd & DP340903 & 1 & c & 0 & ( & 0 & 0 & 0 & 3 & 1 & 2 & 0 & 0 \\
\hline 061 Khawr Habalayn & DP420850 & 2 & 1 & 1 & ( & 0 & 0 & 1 & 4 & 2 & 2 & & 0 \\
\hline 062 Khawr Habalayn & DP430854 & 1 & 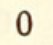 & 1 & & 0 & 0 & 1 & 4 & 2 & 0 & & 0 \\
\hline 063 Dawhat Qabal & DP400800 & 0 & 0 & 0 & & 0 & 0 & 0 & 2 & 0 & 1 & & 0 \\
\hline 064 Dawhat Qabal & DP415782 & 0 & c & 1 & 1 & ( & 0 & 0 & 3 & 1 & 1 & & 0 \\
\hline 065 Ra's Marovi & DP430743 & 1 & 0 & 1 & 1 & ( & 0 & 0 & 4 & 2 & 2 & & 0 \\
\hline $066 \mathrm{Ma}$ awi & DP4C & 1 & 0 & 1 & 1 & ( & 0 & 1 & 4 & 2 & 1 & ( & J \\
\hline 067 Limah & DP4 & 0 & 0 & 1 & 1 & ( & 0 & 0 & 3 & 1 & 2 & & ) \\
\hline 068 Ghubbat Aqabah & 670 & 1 & c & 1 & 1 & 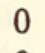 & 0 & 0 & 2 & 1 & 1 & & 0 \\
\hline $069 \mathrm{Gh}$ & DP437677-424680 & 1 & 0 & 1 & 1 & 0 & 0 & 0 & 2 & 1 & 1 & & 0 \\
\hline $070 \mathrm{Ghr}$ & DP395649 & 0 & 0 & 0 & 1 & 0 & 0 & 0 & 1 & 0 & 1 & & 0 \\
\hline 071 Massar & DP375604 & 0 & 0 & 1 & 1 & 0 & 0 & 0 & 4 & 2 & 1 & & ) \\
\hline 072 Musandam SE & DP382589 & 1 & 0 & 1 & 0 & 0 & 0 & 0 & J & 2 & 1 & ) & 0 \\
\hline 073 Musandam SE & DP369563 & 0 & 0 & 1 & 1 & 0 & 0 & 0 & 4 & 2 & 1 & 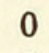 & 0 \\
\hline 074 Sanat & DP357543 & 0 & 0 & 1 & 1 & 0 & 0 & 0 & 4 & 2 & 0 & & 0 \\
\hline 075 Dawhat Haffah & DP310480 & 1 & 0 & 0 & 0 & 0 & 0 & 0 & 2 & 0 & 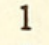 & & 0 \\
\hline 076 Zaghi S & DP276416 & 1 & 0 & 1 & 1 & 0 & 0 & ( & 3 & 1 & 2 & & 0 \\
\hline 077 Ra’s Sawt N & DP2 & 1 & 0 & 1 & 1 & 0 & 0 & 0 & 4 & 1 & 2 & & 0 \\
\hline $078 \mathrm{Daba}$ & DP & 1 & 0 & . & 1 & 0 & 0 & 0 & 4 & 1 & 2 & & 0 \\
\hline 079 Zaghi & 2327 & 0 & 0 & 1 & 1 & 0 & 0 & 0 & & 1 & 3 & 0 & 0 \\
\hline 080 Ra's Sawadi & FM798311 & 1 & 0 & 1 & 1 & 0 & 0 & 0 & & 1 & 1 & & 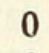 \\
\hline 081 Daymaniyat (Gnl) & EM967380-2 & 2 & 1 & 1 & 1. & 0 & 0 & 1 & & 3 & 2 & 0 & 0 \\
\hline 082 Daymaniyat & EM967380 & 1 & 0 & 1 & c & 0 & 0 & 1 & 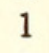 & 1 & 1 & 0 & 0 \\
\hline 083 Daym & EM987366 & 1 & 0 & 1 & c & 0 & 0 & 1 & 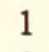 & 2 & 1 & 0 & 0 \\
\hline 084 Daymaniyat & FM000364 & 1 & 0 & 1 & 1 & 0 & 0 & 1 & t & 2 & 1 & 0 & 0 \\
\hline 085 Daym & FM0 & 0 & 0 & 1 & 1 & 0 & 0 & 0 & 1 & 1 & 1 & 0 & 0 \\
\hline 086 Daymaniyat & 6384-093387 & 3 & 1 & 1 & 1 & 0 & 0 & 1 & 2 & 3 & 2 & 0 & 0 \\
\hline 087 Daymaniyat & FM & 2 & 1 & 1 & 0 & 0 & 0 & 0 & 2 & 2 & 1 & 0 & 0 \\
\hline 088 Daymaniyat & FN & 1 & 0 & 1 & 1 & 0 & 0 & 0 & 2 & 3 & 1 & 0 & 0 \\
\hline 089 Daymaniyat & 0387 & 1 & 0 & 1 & 1 & 0 & 0 & 1 & 2 & 2 & 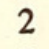 & 0 & 0 \\
\hline 090 Daymaniyat & FM090385 & 1 & 0 & 1 & 1 & 0 & 0 & 0 & 2 & 1 & 1 & 0 & 0 \\
\hline 091 Daymaniyat & FM103386-112390 & 1 & 0 & 1 & 1 & 0 & 0 & 0 & 2 & 2 & 2 & 0 & 0 \\
\hline 092 Daymaniyat & FM106390 & 1 & 0 & 1 & 1 & 0 & 0 & 0 & 2 & 2 & 2 & 0 & 0 \\
\hline 093 Daymaniyat & FM111386 & 1 & 0 & 1 & 1 & 0 & 0 & 0 & 2 & 2 & 1 & 0 & 0 \\
\hline 094 Daymaniyat & FM116393-131391 & 3 & 0 & 1 & 1 & 0 & 0 & 1 & 2 & 3 & 2 & 0 & 0 \\
\hline 095 Daymaniyat & FM1 18388 & 1 & 0 & 1 & 1 & 0 & 0 & 0 & 1 & 1 & 0 & 0 & 0 \\
\hline 096 Daymaniyat & & 1 & 0 & 1 & 1 & 0 & 0 & 1 & 0 & 1 & 2 & 0 & 0 \\
\hline 097 Daymaniyat & 387 & 2 & 0 & 1 & 1 & 0 & 0 & 1 & 2 & 3 & 2 & 0 & 0 \\
\hline 098 Daymaniyat & FM134394-143395 & 3 & 1 & 1 & 1 & 0 & 0 & 1 & 2 & 3 & 2 & 0 & 0 \\
\hline 099 Daymaniyat & FM143395 & 2 & 1 & 1 & 1 & 0 & 0 & 1 & 1 & 2 & 2 & 0 & 0 \\
\hline 100 Daymaniyat & FM150360 & 2 & 0 & 1 & 0 & 0 & 0 & 1 & 3 & 1 & 2 & 0 & 0 \\
\hline 101 Daymaniyat & FM190367-200370 & 1 & 0 & 1 & 1 & 0 & 0 & 1 & 2 & 2 & 1 & 0 & 0 \\
\hline 102 Daymaniyat & FM194371 & 1 & 0 & 1 & 1 & 0 & 0 & 1 & 2 & 2 & 1 & 0 & 0 \\
\hline 103 Fahal Island (Gnl) & FM530195 & 2 & 0 & 1 & 1 & 0 & 1 & 1 & 2 & 2 & 2 & 1 & 0 \\
\hline 104 Fahal Island & FM530194 & 2 & 0 & 1 & 1 & 0 & 0 & 1 & 2 & 1 & 1 & 0 & 0 \\
\hline 105 Fahal Island W & FM529195 & 2 & 0 & 0 & 1 & 0 & 0 & 1 & 1 & 1 & 2 & 0 & 0 \\
\hline
\end{tabular}


APPENDIX 1. Continued

Site Pseud Ano Cos Por P(Syn) Gonio Alv Favia Favit Gonia Pla Leptor Monta Ples Lepta Cyph Echin Cul Phy Gal Par Hyd

\begin{tabular}{|c|c|c|c|c|c|c|c|c|c|c|c|c|c|c|c|c|c|c|c|c|c|}
\hline 052 & 1 & 0 & 1 & 2 & 0 & 2 & 0 & 1 & 3 & 0 & 2 & 0 & 0 & 1 & 2 & 1 & 1 & 0 & 0 & 0 & 1 \\
\hline 053 & 0 & 0 & 0 & 2 & 0 & 1 & 0 & 1 & 3 & 0 & 3 & 0 & 0 & 0 & 3 & 1 & 0 & 0 & 0 & 0 & 0 \\
\hline 054 & 1 & 1 & 1 & 4 & 0 & 2 & 0 & 4 & 2 & 0 & 3 & 0 & 0 & 1 & 3 & 1 & 1 & 1 & 0 & 0 & 1 \\
\hline 055 & 0 & 1 & 0 & 4 & 0 & 1 & 0 & 4 & 2 & 0 & 2 & 0 & 0 & 1 & 2 & 1 & 1 & 0 & 0 & 0 & 1 \\
\hline 056 & 0 & 0 & 0 & 4 & 0 & 1 & 0 & 3 & 1 & 0 & 2 & 0 & 0 & 0 & 1 & 0 & 1 & 0 & 0 & 0 & \\
\hline 057 & 0 & 0 & 1 & 1 & 0 & 0 & 0 & 3 & 2 & 0 & 2 & 0 & 0 & 0 & 2 & 1 & 1 & 0 & 0 & 0 & 0 \\
\hline 058 & 0 & 0 & 1 & 2 & 0 & 1 & 0 & 3 & 1 & 0 & 2 & 0 & 0 & 1 & 3 & 1 & 0 & 0 & 0 & 0 & 0 \\
\hline 059 & 0 & 1 & 1 & 3 & 0 & 1 & 0 & 3 & 0 & 0 & 2 & 0 & 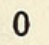 & 1 & 2 & 1 & 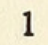 & 0 & 0 & 0 & 1 \\
\hline 060 & 0 & 0 & 1 & 1 & 0 & 1 & 0 & 2 & 1 & 0 & 1 & 0 & 0 & 0 & 1 & 1 & 1 & 0 & 0 & 0 & 0 \\
\hline 061 & 1 & 0 & 1 & 1 & 0 & 1 & 0 & 2 & 2 & 0 & 1 & 0 & 0 & 1 & 2 & 1 & 1 & 1 & 0 & 0 & 1 \\
\hline 062 & 0 & 0 & 1 & 3 & 0 & 2 & 0 & 1 & 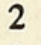 & 0 & 3 & 0 & 0 & 1 & 1 & 0 & 1 & 0 & 0 & 0 & 1 \\
\hline 063 & 0 & 0 & 0 & 1 & 0 & 1 & 0 & 1 & 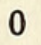 & 0 & 1 & 0 & 0 & 0 & 0 & 0 & 0 & 0 & 0 & 0 & 0 \\
\hline 064 & 0 & 1 & 1 & 3 & 0 & 2 & 0 & 2 & 2 & 0 & 2 & 0 & 0 & 1 & 2 & 2 & 1 & 0 & 0 & 0 & 1 \\
\hline 065 & 0 & 1 & 1 & 2 & 1 & 2 & 0 & 1 & 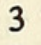 & 0 & 2 & 0 & 0 & 0 & 2 & 1 & 1 & 0 & 0 & 0 & 1 \\
\hline 066 & 0 & 1 & 1 & 2 & 1 & 1 & 0 & 3 & 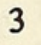 & 0 & 2 & 0 & 0 & 1 & 2 & 2 & 1 & 0 & 0 & 0 & 1 \\
\hline 067 & 0 & 0 & 0 & 1 & 0 & 1 & 0 & 1 & 1 & 0 & ? & 0 & 0 & 0 & 0 & 1 & 1 & 0 & 0 & 0 & 0 \\
\hline 068 & 0 & 0 & 1 & 2 & 0 & 2 & 0 & 0 & 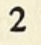 & 0 & - & 0 & 0 & 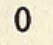 & 2 & 1 & 1 & 0 & 0 & 0 & 0 \\
\hline 069 & 0 & 0 & 1 & 2 & 0 & 2 & 0 & 0 & 2 & 0 & 2 & 0 & 0 & 0 & 2 & 1 & 1 & 0 & 0 & 0 & 0 \\
\hline 070 & 0 & 0 & 0 & 2 & 0 & 0 & 0 & 0 & 2 & 0 & 1 & 0 & 0 & 0 & 1 & 0 & 0 & 0 & 0 & 0 & 0 \\
\hline 071 & 1 & 1 & 1 & 2 & 0 & 1 & 0 & 3 & 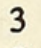 & 0 & 2 & 0 & 0 & 0 & 3 & 1 & 1 & 0 & 0 & 0 & 0 \\
\hline 072 & 1 & 1 & 1 & 3 & 0 & 2 & 0 & 2 & 2 & 0 & 2 & 0 & 0 & 1 & 1 & 1 & 1 & 0 & 0 & 0 & 0 \\
\hline 073 & 0 & 0 & 0 & 3 & 0 & 1 & 0 & 2 & 2 & 0 & 3 & 0 & 0 & 1 & 1 & 1 & 1 & 0 & 0 & 0 & 0 \\
\hline 074 & 0 & 0 & 0 & 3 & 0 & 1 & 0 & 2 & 1 & 0 & 1 & 0 & 0 & 0 & 1 & 1 & 1 & 0 & 0 & 0 & 0 \\
\hline 075 & 0 & 0 & 1 & 2 & 0 & 0 & 0 & 1 & 1 & 0 & 2 & 0 & 0 & 0 & 0 & 1 & 0 & 0 & 0 & 0 & 0 \\
\hline 076 & 1 & 1 & 1 & 3 & 0 & 2 & 0 & 3 & 2 & 0 & 2 & 0 & 0 & 1 & 2 & 1 & 1 & 0 & 0 & 0 & 0 \\
\hline 077 & 1 & 1 & 1 & 2 & 0 & 1 & 0 & 3 & 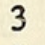 & 0 & 2 & 0 & 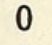 & 1 & 3 & 2 & 1 & 0 & 0 & 0 & 0 \\
\hline 078 & 0 & 1 & 1 & 1 & 0 & 1 & 0 & 2 & 3 & 0 & 2 & 0 & 0 & 0 & 2 & 1 & 1 & 0 & 0 & 0 & 0 \\
\hline 079 & 1 & 1 & 1 & 1 & 0 & 1 & 1 & 3 & 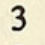 & 0 & 2 & 0 & 0 & 1 & 2 & 1 & 1 & 0 & 0 & 0 & 1 \\
\hline 080 & 0 & 1 & 1 & 2 & 0 & 1 & 0 & 1 & 3 & 0 & 1 & 0 & 0 & 0 & 1 & 1 & 0 & 0 & 0 & 0 & 0 \\
\hline 081 & 1 & 1 & 2 & 3 & 0 & 2 & 0 & 1 & 3 & 0 & 1 & 0 & 0 & 1 & 3 & 2 & 2 & 1 & 0 & 1 & 1 \\
\hline 082 & 0 & 0 & 1 & 1 & 0 & 1 & 0 & 1 & 1 & 0 & 1 & 0 & 0 & 1 & 1 & 0 & 0 & 0 & 0 & 0 & 0 \\
\hline 083 & 0 & 0 & 1 & 1 & 0 & 1 & 0 & 0 & 1 & 0 & 1 & $c$ & 0 & 1 & 1 & 0 & 1 & 0 & 0 & 0 & 0 \\
\hline 084 & 0 & 0 & 1 & 2 & 0 & 1 & 0 & 0 & 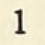 & 0 & 1 & 0 & $v$ & 1 & 1 & 0 & 1 & 0 & 0 & 1 & 0 \\
\hline 085 & 0 & 0 & 0 & 2 & 0 & 1 & 0 & 0 & 0 & 0 & 1 & 0 & 0 & 0 & 0 & 0 & 0 & 0 & 0 & 0 & 0 \\
\hline 086 & 0 & 0 & 1 & 2 & 0 & 2 & 0 & 2 & 2 & 0 & 1 & 0 & 0 & 1 & 3 & 1 & 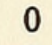 & 1 & 0 & 1 & 1 \\
\hline 087 & 0 & 0 & 0 & 2 & 0 & 2 & 0 & 1 & 2 & 0 & 1 & 0 & 0 & 1 & 2 & 1 & ) & 0 & 0 & 0 & 0 \\
\hline 088 & 0 & 0 & 0 & 2 & 0 & 2 & 0 & 0 & 2 & 0 & 1 & 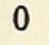 & 0 & 1 & 2 & 1 & 0 & 0 & 0 & 0 & 1 \\
\hline 089 & 0 & 0 & 1 & 2 & 0 & 1 & 0 & 2 & 2 & 0 & 1 & $c$ & 0 & 1 & 1 & 1 & 0 & 0 & 0 & 1 & 0 \\
\hline 090 & 0 & 0 & 0 & 1 & 0 & 1 & 0 & 0 & 0 & 0 & 1 & 0 & 0 & 0 & 0 & 0 & 0 & 0 & 0 & 0 & 0 \\
\hline 091 & 0 & 0 & 0 & 2 & 0 & 2 & 0 & 1 & 2 & 0 & 1 & 0 & 0 & 1 & 1 & 1 & 0 & 0 & 0 & 1 & 0 \\
\hline 092 & 0 & 0 & 0 & 1 & 0 & 2 & 0 & 1 & 1 & 0 & 1 & 0 & 0 & 1 & 1 & 1 & 0 & 0 & 0 & 1 & 0 \\
\hline 093 & 0 & 0 & 0 & 2 & 0 & 1 & 0 & 0 & 1 & 0 & 1 & 0 & 0 & 0 & 1 & 1 & 0 & 0 & 0 & 0 & 0 \\
\hline 094 & 0 & 0 & 1 & 2 & 0 & 2 & 0 & 1 & 2 & 0 & 2 & 0 & 0 & 1 & 2 & 1 & 0 & 1 & 0 & 1 & 1 \\
\hline 095 & 0 & 0 & 0 & 1 & 0 & 0 & 0 & 1 & 0 & 0 & 2 & 0 & 0 & 0 & 1 & 1 & 0 & 0 & 0 & 0 & 0 \\
\hline 096 & 0 & 0 & 1 & 1 & 0 & 2 & 0 & 1 & 2 & 0 & 1 & 0 & 0 & 1 & 1 & 1 & 0 & 0 & 0 & 1 & 1 \\
\hline 097 & 0 & 0 & 1 & 2 & 0 & 2 & 0 & 1 & 2 & 0 & 1 & 0 & 0 & 0 & 2 & 1 & 0 & 1 & 0 & 0 & 0 \\
\hline 098 & 0 & 0 & 2 & 2 & 0 & 1 & 0 & 1 & 2 & 0 & 1 & 0 & 0 & 1 & 3 & 1 & 1 & 0 & 0 & 0 & 1 \\
\hline 099 & 0 & 0 & 2 & 1 & 0 & 1 & 0 & 1 & 2 & 0 & 1 & 0 & 0 & 1 & 3 & 1 & 1 & 0 & 0 & 0 & 1 \\
\hline 100 & 1 & 1 & 2 & 1 & 0 & 2 & 0 & 1 & 3 & 0 & 1 & 0 & 0 & 1 & 2 & 2 & 1 & 1 & 0 & 0 & 1 \\
\hline 101 & 0 & 0 & 1 & 2 & 0 & 1 & 0 & 1 & 2 & 0 & 1 & 0 & 0 & 1 & 2 & 1 & 1 & 0 & 0 & 0 & 0 \\
\hline 102 & 0 & 0 & 1 & 1 & 0 & 1 & 0 & 1 & 2 & 0 & 1 & 0 & 0 & 1 & 2 & 1 & 0 & 0 & 0 & 0 & 0 \\
\hline 103 & 1 & 1 & 2 & 2 & 0 & 2 & 0 & 2 & 4 & 0 & 1 & 0 & 0 & 1 & 1 & 1 & 1 & 1 & 1 & 1 & 1 \\
\hline 104 & 0 & 1 & 0 & 2 & 0 & 1 & 0 & 1 & 4 & 0 & 1 & 0 & 0 & 1 & 0 & 1 & 0 & 0 & 0 & 0 & 0 \\
\hline 105 & 0 & 1 & 1 & 1 & 0 & 1 & 0 & 2 & 3 & 0 & 1 & 0 & 0 & 1 & 0 & 1 & 1 & 1 & 0 & & 0 \\
\hline
\end{tabular}


APPENDIX 1. Continued

Site Bla Sym Aca Echinoph Oxy Euph Parac Poly Heteroc Cary Bal Rhiz Den Tub Tur Heterop Tubip Mille Stylas Unid G S

$\begin{array}{lllllllllllllllllllllll}052 & 0 & 1 & 1 & 1 & 1 & 0 & 0 & 0 & 0 & 0 & 0 & 0 & 0 & 1 & 0 & 0 & 0 & 0 & 0 & 0 & 26 & 36\end{array}$

$\begin{array}{lllllllllllllllllllllll}053 & 0 & 1 & 1 & 1 & 0 & 0 & 0 & 0 & 0 & 0 & 0 & 0 & 0 & 1 & 1 & 0 & 0 & 0 & 0 & 0 & 16 & 24\end{array}$

$\begin{array}{llllllllllllllllllllllll}054 & 1 & 2 & 1 & 1 & 2 & 0 & 0 & 0 & 0 & 0 & 0 & 0 & 1 & 1 & 0 & 0 & 0 & 0 & 0 & 0 & 31 & 53\end{array}$

$\begin{array}{lllllllllllllllllllllllll}055 & 1 & 1 & 1 & 1 & 1 & 0 & 0 & 0 & 0 & 0 & 0 & 0 & 0 & 1 & 0 & 0 & 0 & 0 & 0 & 0 & 25 & 39\end{array}$

$\begin{array}{llllllllllllllllllllllll}056 & 1 & 1 & 0 & 1 & 1 & 0 & 0 & 0 & 0 & 0 & 0 & 0 & 0 & 1 & 0 & 0 & 0 & 0 & 0 & 0 & 20 & 31\end{array}$

$\begin{array}{lllllllllllllllllllllll}057 & 1 & 1 & 1 & 1 & 1 & 0 & 0 & 0 & 0 & 0 & 0 & 0 & 0 & 1 & 0 & 0 & 0 & 0 & 0 & 0 & 20 & 28\end{array}$

$\begin{array}{llllllllllllllllllllllll}058 & 1 & 1 & 1 & 1 & 1 & 0 & 0 & 0 & 0 & 0 & 0 & 0 & 0 & 0 & 0 & 0 & 0 & 0 & 0 & 0 & 21 & 31\end{array}$

$\begin{array}{lllllllllllllllllllllll}059 & 0 & 1 & 1 & 1 & 1 & 0 & 0 & 0 & 0 & 0 & 0 & 0 & 0 & 0 & 0 & 0 & 0 & 0 & 0 & 0 & 23 & 34\end{array}$

$\begin{array}{llllllllllllllllllllllllll}060 & 0 & 1 & 1 & 1 & 1 & 0 & 0 & 0 & 0 & 0 & 0 & 0 & 0 & 0 & 0 & 0 & 0 & 0 & 0 & 0 & 18 & 22\end{array}$

$\begin{array}{lllllllllllllllllllllll}061 & 1 & 2 & 1 & 1 & 2 & 0 & 0 & 0 & 0 & 0 & 0 & 0 & 1 & 0 & 0 & 0 & 0 & 0 & 0 & 0 & 28 & 40\end{array}$

$\begin{array}{lllllllllllllllllllllll}062 & 0 & 1 & 1 & 1 & 1 & 0 & 0 & 0 & 0 & 0 & 0 & 0 & 0 & 1 & 0 & 0 & 0 & 0 & 0 & 0 & 22 & 33\end{array}$

$\begin{array}{llllllllllllllllllllllll}063 & 0 & 1 & 0 & 1 & 0 & 0 & 0 & 0 & 0 & 0 & 0 & 0 & 0 & 0 & 0 & 0 & 0 & 0 & 0 & 0 & 9 & 10\end{array}$

$\begin{array}{lllllllllllllllllllllll}064 & 1 & 1 & 1 & 1 & 1 & 0 & 0 & 0 & 0 & 0 & 0 & 0 & 0 & 0 & 0 & 0 & 0 & 0 & 0 & 0 & 23 & 34\end{array}$

$\begin{array}{llllllllllllllllllllllll}065 & 0 & 1 & 1 & 1 & 1 & 0 & 0 & 0 & 0 & 0 & 0 & 0 & 0 & 2 & 1 & 0 & 0 & 0 & 0 & 0 & 26 & 39\end{array}$

$\begin{array}{llllllllllllllllllllllll}066 & 0 & 1 & 1 & 1 & 0 & 0 & 0 & 0 & 0 & 0 & 0 & 0 & 0 & 0 & 1 & 0 & 0 & 0 & 0 & 0 & 25 & 38\end{array}$

$\begin{array}{lllllllllllllllllllllll}067 & 0 & 1 & 1 & 1 & 0 & 0 & 0 & 0 & 0 & 0 & 0 & 0 & 0 & 0 & 0 & 0 & 0 & 0 & 0 & 0 & 15 & 19\end{array}$

$\begin{array}{lllllllllllllllllllllll}068 & 0 & 1 & 1 & 1 & 1 & 0 & 0 & 0 & 0 & 0 & 0 & 0 & 0 & 1 & 1 & 0 & 0 & 0 & 0 & 0 & 21 & 27\end{array}$

$\begin{array}{lllllllllllllllllllllll}069 & 0 & 1 & 1 & 1 & 1 & 0 & 0 & 0 & 0 & 0 & 0 & 0 & 0 & 1 & 1 & 0 & 0 & 0 & 0 & 0 & 21 & 27\end{array}$

$\begin{array}{llllllllllllllllllllllll}070 & 0 & 1 & 1 & 0 & 0 & 0 & 0 & 0 & 0 & 0 & 0 & 0 & 0 & 0 & 0 & 0 & 0 & 0 & 0 & 0 & 9 & 11\end{array}$

$\begin{array}{lllllllllllllllllllllll}071 & 0 & 1 & 1 & 1 & 1 & 0 & 0 & 0 & 0 & 0 & 0 & 0 & 0 & 0 & 0 & 0 & 0 & 0 & 0 & 0 & 21 & 34\end{array}$

$\begin{array}{lllllllllllllllllllllllll}072 & 1 & 1 & 1 & 1 & 0 & 0 & 0 & 0 & 0 & 0 & 0 & 0 & 0 & 1 & 1 & 0 & 0 & 0 & 0 & 0 & 24 & 33\end{array}$

$\begin{array}{lllllllllllllllllllllll}073 & 0 & 1 & 1 & 1 & 0 & 0 & 0 & 0 & 0 & 0 & 0 & 0 & 0 & 0 & 0 & 0 & 0 & 0 & 0 & 0 & 18 & 29\end{array}$

$\begin{array}{llllllllllllllllllllllll}074 & 0 & 1 & 1 & 1 & 0 & 0 & 0 & 0 & 0 & 0 & 0 & 0 & 0 & 0 & 0 & 0 & 0 & 0 & 0 & 0 & 15 & 22\end{array}$

$\begin{array}{llllllllllllllllllllllll}075 & 0 & 0 & 0 & 0 & 0 & 0 & 0 & 0 & 0 & 0 & 0 & 0 & 0 & 0 & 0 & 0 & 0 & 0 & 0 & 0 & 9 & 12\end{array}$

$\begin{array}{lllllllllllllllllllllll}076 & 0 & 1 & 1 & 1 & 1 & 0 & 0 & 0 & 0 & 0 & 0 & 0 & 0 & 0 & 1 & 0 & 0 & 0 & 0 & 0 & 25 & 37\end{array}$

$\begin{array}{lllllllllllllllllllllll}077 & 0 & 1 & 1 & 1 & 1 & 0 & 0 & 0 & 0 & 0 & 0 & 0 & 0 & 0 & 1 & 0 & 0 & 0 & 0 & 0 & 24 & 37\end{array}$

$\begin{array}{lllllllllllllllllllllllll}078 & 0 & 1 & 1 & 1 & 0 & 0 & 0 & 0 & 0 & 0 & 0 & 0 & 0 & 0 & 1 & 0 & 0 & 0 & 0 & 0 & 19 & 28\end{array}$

$\begin{array}{lllllllllllllllllllllllll}079 & 0 & 1 & 1 & 1 & 1 & 0 & 0 & 0 & 0 & 0 & 0 & 0 & 0 & 0 & 1 & 0 & 0 & 0 & 0 & 0 & 25 & 34\end{array}$

$\begin{array}{lllllllllllllllllllllll}080 & 0 & 1 & 1 & 1 & 0 & 0 & 0 & 0 & 0 & 0 & 0 & 0 & 0 & 0 & 1 & 0 & 0 & 0 & 0 & 0 & 20 & 24\end{array}$

$\begin{array}{lllllllllllllllllllllll}081 & 2 & 1 & 1 & 1 & 1 & 0 & 0 & 1 & 0 & 0 & 0 & 0 & 1 & 1 & 3 & 0 & 0 & 0 & 0 & 0 & 34 & 53\end{array}$

$\begin{array}{lllllllllllllllllllllll}082 & 0 & 1 & 0 & 1 & 0 & 0 & 0 & 0 & 0 & 0 & 0 & 0 & 0 & 0 & 1 & 0 & 0 & 0 & 0 & 0 & 17 & 17\end{array}$

$\begin{array}{llllllllllllllllllllllllll}083 & 0 & 1 & 0 & 0 & 0 & 0 & 0 & 0 & 0 & 0 & 0 & 0 & 0 & 0 & 0 & 0 & 0 & 0 & 0 & 0 & 15 & 16\end{array}$

$\begin{array}{lllllllllllllllllllllll}084 & 0 & 1 & 0 & 0 & 0 & 0 & 0 & 0 & 0 & 0 & 0 & 0 & 1 & 0 & 0 & 0 & 0 & 0 & 0 & 0 & 19 & 21\end{array}$

$\begin{array}{llllllllllllllllllllllllll}085 & 0 & 1 & 0 & 0 & 0 & 0 & 0 & 0 & 0 & 0 & 0 & 0 & 1 & 0 & 0 & 0 & 0 & 0 & 0 & 0 & 11 & 12\end{array}$

$\begin{array}{lllllllllllllllllllllll}086 & 1 & 1 & 1 & 1 & 1 & 0 & 0 & 0 & 0 & 0 & 0 & 0 & 1 & 1 & 1 & 0 & 0 & 0 & 0 & 0 & 29 & 41\end{array}$

$\begin{array}{llllllllllllllllllllllll}087 & 1 & 1 & 0 & 1 & 1 & 0 & 0 & 0 & 0 & 0 & 0 & 0 & 1 & 0 & 0 & 0 & 0 & 0 & 0 & 0 & 19 & 26\end{array}$

$\begin{array}{llllllllllllllllllllllll}088 & 0 & 1 & 0 & 0 & 0 & 0 & 0 & 0 & 0 & 0 & 0 & 0 & 0 & 1 & 1 & 0 & 0 & 0 & 0 & 0 & 18 & 25\end{array}$

$\begin{array}{lllllllllllllllllllllll}089 & 0 & 1 & 1 & 1 & 0 & 0 & 0 & 0 & 0 & 0 & 0 & 0 & 1 & 0 & 0 & 0 & 0 & 0 & 0 & 0 & 22 & 28\end{array}$

$\begin{array}{llllllllllllllllllllllll}090 & 0 & 1 & 0 & 0 & 0 & 0 & 0 & 0 & 0 & 0 & 0 & 0 & 0 & 0 & 0 & 0 & 0 & 0 & 0 & 0 & 11 & 12\end{array}$

$\begin{array}{lllllllllllllllllllllll}091 & 0 & 1 & 1 & 1 & 0 & 0 & 0 & 0 & 0 & 0 & 0 & 0 & 1 & 1 & 1 & 0 & 0 & 0 & 0 & 0 & 22 & 28\end{array}$

$\begin{array}{llllllllllllllllllllllll}092 & 0 & 1 & 1 & 1 & 0 & 0 & 0 & 0 & 0 & 0 & 0 & 0 & 1 & 1 & 0 & 0 & 0 & 0 & 0 & 0 & 21 & 25\end{array}$

$\begin{array}{llllllllllllllllllllllll}093 & 0 & 1 & 0 & 0 & 0 & 0 & 0 & 0 & 0 & 0 & 0 & 0 & 0 & 0 & 1 & 0 & 0 & 0 & 0 & 0 & 15 & 18\end{array}$

$\begin{array}{llllllllllllllllllllllll}094 & 0 & 1 & 1 & 1 & 0 & 0 & 0 & 0 & 0 & 0 & 0 & 0 & 1 & 0 & 1 & 0 & 0 & 0 & 0 & 0 & 26 & 37\end{array}$

$\begin{array}{lllllllllllllllllllllll}095 & 0 & 0 & 0 & 0 & 0 & 0 & 0 & 0 & 0 & 0 & 0 & 0 & 0 & 0 & 1 & 0 & 0 & 0 & 0 & 0 & 12 & 13\end{array}$

$\begin{array}{lllllllllllllllllllllll}096 & 0 & 1 & 1 & 0 & 0 & 0 & 0 & 0 & 0 & 0 & 0 & 0 & 1 & 0 & 1 & 0 & 0 & 0 & 0 & 0 & 22 & 25\end{array}$

$\begin{array}{lllllllllllllllllllllll}097 & 1 & 1 & 1 & 1 & 0 & 0 & 0 & 0 & 0 & 0 & 0 & 0 & 0 & 0 & 1 & 0 & 0 & 0 & 0 & 0 & 23 & 32\end{array}$

$\begin{array}{llllllllllllllllllllllll}098 & 1 & 1 & 0 & 1 & 0 & 0 & 0 & 0 & 0 & 0 & 0 & 0 & 1 & 1 & 2 & 0 & 0 & 0 & 0 & 0 & 26 & 38\end{array}$

$\begin{array}{lllllllllllllllllllllll}099 & 1 & 1 & 0 & 1 & 0 & 0 & 0 & 0 & 0 & 0 & 0 & 0 & 1 & 1 & 1 & 0 & 0 & 0 & 0 & 0 & 26 & 33\end{array}$

$\begin{array}{llllllllllllllllllllllll}100 & 0 & 1 & 1 & 1 & 1 & 0 & 0 & 1 & 0 & 0 & 0 & 0 & 1 & 0 & 1 & 0 & 0 & 0 & 0 & 0 & 28 & 38\end{array}$

$\begin{array}{lllllllllllllllllllllll}101 & 0 & 1 & 0 & 0 & 0 & 0 & 0 & 0 & 0 & 0 & 0 & 0 & 0 & 0 & 2 & 0 & 0 & 0 & 0 & 0 & 20 & 26\end{array}$

$\begin{array}{lllllllllllllllllllllll}102 & 0 & 1 & 0 & 0 & 0 & 0 & 0 & 0 & 0 & 0 & 0 & 0 & 0 & 0 & 1 & 0 & 0 & 0 & 0 & 0 & 19 & 23\end{array}$

$\begin{array}{lllllllllllllllllllllllll}103 & 1 & 1 & 1 & 1 & 1 & 0 & 1 & 1 & 1 & 0 & 1 & 1 & 1 & 1 & 1 & 1 & 0 & 0 & 0 & 0 & 41 & 52\end{array}$

$\begin{array}{lllllllllllllllllllllllll}104 & 0 & 1 & 1 & 1 & 0 & 0 & 0 & 0 & 0 & 0 & 0 & 0 & 0 & 0 & 1 & 0 & 0 & 0 & 0 & 0 & 20 & 26\end{array}$

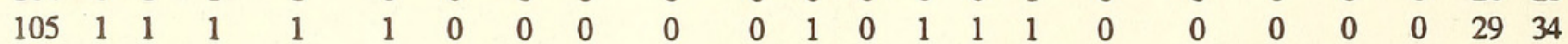


APPENDIX 1. Continued

Site Site Name Map Coordinates Psam Styloc Poc Stylop Ser Mad Ast Acr Monti Pav Leptos Gar Sid

\begin{tabular}{|c|c|}
\hline 106 Fahal, Shallow Rf & FM536199 \\
\hline 107 Fahal, Deep Reef & FM535206 \\
\hline 108 Ra's al Hamra N & FM527162 \\
\hline 109 Darsayt & FM580148 \\
\hline 110 Aint East & FM588146 \\
\hline $111 \mathrm{Kalbuh}$ & FM617136 \\
\hline 112 Muscat Island E & FM632137 \\
\hline 113 Muscat Island & FM634132 \\
\hline 114 Cemetary Bay & FM633122 \\
\hline 115 Cemetary Bay & FM635119 \\
\hline 116 Cemetary Bay & FM637121 \\
\hline 117 Cemetary Bay S & FM638117 \\
\hline 118 Cat Island & FM642092 \\
\hline 119 Al Bustan & FM653075 \\
\hline 120 Qantab & FM668068 \\
\hline 121 Jissah (Gnl) & FM685060 \\
\hline 122 Jissah & FM676064 \\
\hline 123 Jissah & FM682056 \\
\hline 124 Jissah & FM684057 \\
\hline 125 Jissah & FM686053 \\
\hline 126 Jissah & FM687050 \\
\hline 127 Khayran (Gnl) & FM770025 \\
\hline 128 Khayran & FM757029 \\
\hline 129 Khayran & FM758022 \\
\hline 130 Khayran & FM760022 \\
\hline 131 Khayran & FM762023 \\
\hline 132 Khayran & FM783026 \\
\hline 133 Khayran & FM784024 \\
\hline 134 Khayran & FM784021 \\
\hline 135 Ra's Khayran & FM792014 \\
\hline 136 Ra's Khayran & FM794014 \\
\hline 137 Ash Shaykh S & FM796006 \\
\hline 138 Ash Shaykh SE & FM798008 \\
\hline 139 As Sifah N & FL816972 \\
\hline 140 As Sifah & FL844885 \\
\hline 141 Ra's Abu Da'ud & FL960800 \\
\hline 142 Bimmah N & GL185450 \\
\hline 143 Makalla Wabar & GL293304 \\
\hline 144 Ra's N Qalhat & GL380185 \\
\hline 145 Bi’r Bira' W & GL520045 \\
\hline 146 Bi'r Bira' & GL533033 \\
\hline 147 S Shiya & GK755959 \\
\hline 148 Barr al Hikman & FH520520 \\
\hline 149 Barr al Hikman & FH480500 \\
\hline 150 Barr al Hikman & FH420528 \\
\hline 151 Ra's Kanasah & FH270530 \\
\hline 152 Ra’s Shajrit & FH244594-246600 \\
\hline 153 Jazirat Abb & FH215672 \\
\hline 154 Gulf of Masirah & FH300480 \\
\hline 155 Bandar Qinqari E & BD891827-890812 \\
\hline 156 Wadi Bayt Said E & BD828795 \\
\hline 157 Wadi Aingalf E3 & BD756770 \\
\hline 158 Wadi Aingalf E2 & BD752769 \\
\hline 159 Wadi Aingalf E1 & BD748767 \\
\hline
\end{tabular}

\begin{tabular}{|c|c|c|c|c|c|c|c|c|c|c|c|}
\hline & 0 & 0 & 0 & 0 & 1 & 0 & 0 & 2 & 1 & 1 & 0 \\
\hline ) & 0 & 0 & 0 & 0 & 1 & 0 & 0 & 0 & 0 & 1 & 0 \\
\hline & 0 & 0 & 0 & 0 & 0 & 0 & 0 & 0 & 0 & 0 & 0 \\
\hline & 0 & 1 & 1 & & 0 & 0 & 2 & 1 & 0 & & 0 \\
\hline & 0 & 1 & 1 & & 0 & 0 & 2 & 1 & 1 & & 0 \\
\hline & 0 & 1 & 1 & 0 & 0 & 0 & 3 & 2 & 1 & & 0 \\
\hline & 0 & 1 & 0 & 0 & 0 & 0 & 1 & 0 & 0 & & 0 \\
\hline & 0 & 1 & 1 & 0 & 0 & 0 & 1 & 1 & 1 & & 0 \\
\hline & 0 & 2 & 2 & 0 & 0 & 0 & 3 & 1 & 2 & & 0 \\
\hline & 0 & 1 & 1 & & 0 & 0 & 2 & 0 & 1 & & 0 \\
\hline & 0 & 1 & 1 & 0 & 0 & 1 & 2 & 0 & 1 & & 0 \\
\hline & 1 & 0 & 0 & 0 & 0 & 0 & 1 & 0 & 0 & & 0 \\
\hline 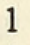 & 0 & 1 & 1 & 0 & 0 & 1 & 2 & 1 & 2 & & 0 \\
\hline 1 & 0 & 1 & 1 & 0 & 0 & 1 & 2 & 1 & 1 & & 0 \\
\hline 1 & 0 & 1 & 1 & 0 & 0 & 1 & 2 & 1 & 2 & & 0 \\
\hline 2 & 0 & 1 & 1 & 0 & 0 & 1 & 2 & 1 & 2 & 0 & 0 \\
\hline & 0 & 1 & 1 & 0 & 0 & 1 & 2 & 1 & 0 & & 0 \\
\hline & 0 & 1 & 1 & 0 & 0 & 0 & 2 & 0 & 0 & & 0 \\
\hline ) & 0 & 1 & 0 & 0 & 0 & 1 & 1 & 1 & 1 & & 0 \\
\hline 1 & 0 & 1 & 1 & 0 & 0 & 0 & 2 & 0 & 0 & & 0 \\
\hline 1 & 0 & 1 & 1 & 0 & 0 & 0 & 2 & 1 & 1 & & 0 \\
\hline 2 & 1 & 1 & 1 & 0 & 0 & 1 & 6 & 2 & 2 & & 0 \\
\hline 1 & 0 & 1 & 1 & 0 & 0 & 1 & 2 & 1 & 2 & & 0 \\
\hline 1 & 0 & 1 & 1 & 0 & 0 & 1 & 6 & 1 & 1 & & 0 \\
\hline 1 & 0 & 1 & 1 & 0 & 0 & 0 & 2 & 1 & 1 & & 0 \\
\hline 1 & 0 & 1 & 1 & 0 & 0 & 1 & 4 & 2 & 2 & U & 0 \\
\hline 0 & 0 & 1 & 1 & 0 & 0 & 0 & 1 & 1 & 0 & & 0 \\
\hline 1 & 0 & 1 & 1 & 0 & 0 & 1 & 1 & 0 & 2 & 0 & 0 \\
\hline 1 & 0 & 0 & 1 & 0 & 0 & 1 & 2 & 2 & 2 & 0 & 0 \\
\hline 1 & 0 & 1 & 1 & 0 & 0 & 1 & 1 & 1 & 1 & 0 & 0 \\
\hline 2 & 0 & 1 & 1 & 0 & 0 & 1 & 2 & 2 & 2 & 0 & 0 \\
\hline 1 & 0 & 1 & 1 & 0 & 0 & 1 & 2 & 1 & 0 & 0 & 0 \\
\hline 1 & 0 & 1 & 1 & 0 & 0 & 1 & 2 & 1 & 1 & 1 & 0 \\
\hline 0 & 0 & 1 & 0 & 0 & 0 & 0 & 1 & 1 & 0 & 0 & 0 \\
\hline 0 & 0 & 1 & 1 & 0 & 0 & 1 & 2 & 1 & 0 & 0 & 0 \\
\hline 2 & 0 & 1 & 1 & 0 & 0 & 1 & 2 & 2 & 2 & 0 & 0 \\
\hline 0 & 0 & 0 & 0 & 0 & 0 & 1 & 3 & 1 & 0 & 0 & 0 \\
\hline 2 & 0 & 1 & 0 & 0 & 0 & 1 & 3 & 2 & 1 & 0 & 0 \\
\hline 1 & 1 & 1 & 0 & 0 & 0 & 1 & 2 & 1 & 1 & 0 & 0 \\
\hline 2 & 0 & 1 & 1 & 0 & 0 & 1 & 3 & 3 & 1 & 0 & 0 \\
\hline 1 & 0 & 1 & 1 & 0 & 0 & 1 & 2 & 1 & 1 & 0 & 0 \\
\hline 2 & 0 & 1 & 0 & 0 & 0 & 1 & 2 & 2 & 0 & 0 & 0 \\
\hline 1 & 0 & 1 & 1 & 0 & 0 & 0 & 2 & 3 & 1 & 0 & 0 \\
\hline 0 & 0 & 0 & 1 & 0 & 0 & 0 & 1 & 3 & 0 & 0 & 0 \\
\hline 0 & 0 & 0 & 1 & 0 & 0 & 0 & 1 & 2 & 0 & 0 & 0 \\
\hline 0 & 0 & 0 & 1 & 0 & 0 & 0 & 1 & 0 & 1 & 0 & 0 \\
\hline 0 & 0 & 0 & 1 & 0 & 0 & 0 & 1 & 1 & 0 & 0 & 0 \\
\hline 0 & 0 & 0 & 0 & 0 & 0 & 0 & 1 & 0 & 0 & 0 & 0 \\
\hline 1 & 0 & 1 & 1 & 0 & 0 & 1 & 2 & 3 & 1 & 0 & 0 \\
\hline 0 & 0 & 0 & 1 & 0 & 0 & 1 & 2 & 2 & 1 & 0 & 1 \\
\hline 1 & 0 & 0 & 1 & 0 & 0 & 0 & 3 & 2 & 1 & 1 & 1 \\
\hline 1 & 0 & 0 & 1 & 0 & 0 & 0 & 1 & 2 & 3 & 1 & 1 \\
\hline 2 & 0 & 0 & 1 & 0 & 0 & 0 & 2 & 2 & 1 & 0 & 1 \\
\hline 1 & 0 & 0 & 1 & 0 & 0 & 0 & 2 & 2 & 2 & 1 & \\
\hline
\end{tabular}


APPENDIX 1. Continued

Site Pseud Ano Cos Por P(Syn) Gonio Alv Favia Favit Gonia Pla Leptor Monta Ples Lepta Cyph Echin Cul Phy Gal Par Hyd

\begin{tabular}{|c|c|c|c|c|c|c|c|c|c|c|c|c|c|c|c|c|c|c|c|c|c|c|c|}
\hline 106 & 1 & 1 & 2 & 1 & 0 & 2 & 0 & 1 & 1 & 0 & 1 & 0 & 0 & & 1 & 1 & 1 & 0 & & 0 & 0 & 0 & 1 \\
\hline 107 & 0 & 0 & 1 & 0 & 0 & 0 & 0 & 0 & 0 & 0 & 0 & 0 & 0 & & 0 & 0 & 0 & ( & & 1 & 1 & 0 & 0 \\
\hline 108 & 1 & 1 & 1 & 1 & 0 & 1 & 0 & 3 & 2 & 0 & 1 & 0 & 0 & & 1 & 0 & 0 & & & 0 & 0 & 0 & 1 \\
\hline 09 & 0 & 1 & 1 & 1 & 0 & 0 & 0 & 1 & 2 & 0 & 1 & 0 & 0 & & 0 & 1 & & & & 0 & 0 & 0 & \\
\hline 10 & 0 & 1 & 1 & 2 & 0 & 1 & 0 & 1 & 3 & 0 & 1 & 0 & 0 & & 2 & 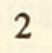 & & & & 0 & 0 & 0 & \\
\hline 11 & 0 & 1 & 0 & 2 & 0 & 1 & 0 & 0 & 2 & 0 & 2 & 0 & 0 & & . & 0 & & & & 0 & 0 & 0 & 0 \\
\hline 12 & 0 & 0 & 0 & 1 & 0 & 1 & 0 & 0 & 0 & 0 & 1 & 0 & 0 & & 1 & 0 & 1 & & & 0 & 0 & 0 & 0 \\
\hline 13 & 0 & 1 & 1 & 1 & 0 & 1 & 0 & 0 & 1 & 0 & 1 & 0 & 0 & & 1 & 0 & 1 & & & 0 & 0 & 0 & 0 \\
\hline 14 & 0 & 0 & 0 & 2 & 0 & 1 & 0 & 0 & 0 & 0 & 1 & 0 & 0 & & 0 & 1 & 1 & & & 0 & 0 & 1 & 0 \\
\hline 115 & 0 & 0 & 0 & 2 & 0 & 0 & 0 & 0 & 0 & 0 & 1 & 0 & 0 & & 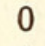 & 1 & 0 & & & 0 & 0 & 0 & 0 \\
\hline 16 & 1 & 1 & 1 & 2 & 0 & 2 & 0 & 0 & 2 & 0 & 2 & 0 & 0 & & 1 & 2 & & & & 1 & 0 & 0 & \\
\hline 17 & 0 & 1 & 0 & 1 & 0 & 1 & 0 & 0 & 0 & 0 & 1 & 0 & 0 & & U & 1 & & & & 0 & 0 & 0 & \\
\hline 18 & 1 & 1 & 2 & 2 & 0 & 2 & 0 & 1 & 2 & 0 & 1 & 0 & 0 & & 1 & 2 & & & & 0 & 0 & 0 & \\
\hline 19 & 0 & 1 & 1 & 1 & 0 & 2 & 0 & 0 & 2 & 0 & 1 & 0 & 0 & & 1 & 1 & 1 & & & 0 & 0 & 0 & 0 \\
\hline 20 & 0 & 1 & 1 & 1 & 0 & 2 & 0 & 1 & 2 & 0 & 1 & 0 & 0 & & 1 & 1 & 1 & & & 0 & 0 & 1 & 0 \\
\hline 21 & 1 & 1 & 1 & 2 & 0 & 2 & 0 & 3 & 2 & 0 & 1 & 0 & 0 & & 1 & 3 & & & & 1 & 0 & 1 & \\
\hline 22 & 0 & 1 & 1 & 1 & 0 & 2 & 0 & 1 & 2 & 0 & 1 & 0 & 0 & & 1 & 3 & 1 & & & 0 & 0 & 0 & 0 \\
\hline 123 & 0 & 0 & 0 & 2 & 0 & 1 & 0 & 0 & 0 & 0 & 1 & 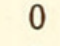 & 0 & & 1 & 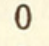 & 0 & & & 0 & 0 & 0 & \\
\hline 24 & 1 & 1 & 1 & 2 & 0 & 2 & 0 & 2 & 1 & 0 & 1 & 0 & 0 & & 1 & 1 & & & & 0 & 0 & 0 & \\
\hline 25 & 0 & 0 & 0 & 1 & 0 & 1 & 0 & 1 & 1 & 0 & 1 & 0 & 0 & & 0 & 1 & & & & 0 & 0 & 1 & 0 \\
\hline 26 & 0 & 0 & 0 & 2 & 0 & 1 & 0 & 3 & 1 & 0 & 1 & 0 & 0 & & 0 & 1 & 1 & & & 0 & 0 & 1 & 0 \\
\hline 127 & 1 & 1 & 2 & 2 & 0 & 2 & 0 & 2 & 3 & 0 & 1 & 0 & 0 & & 1 & 3 & & & & 0 & 0 & 1 & 1 \\
\hline 128 & 0 & 1 & 1 & 1 & 0 & 2 & 0 & 1 & 3 & 0 & 1 & 0 & 0 & & 1 & 2 & 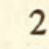 & & & 0 & 0 & 0 & 0 \\
\hline 129 & 0 & 1 & 1 & 2 & 0 & 1 & 0 & 1 & 1 & 0 & 1 & 0 & 0 & & 1 & 1 & 1 & & & 0 & 0 & 0 & 0 \\
\hline 130 & 0 & 1 & 1 & 1 & 0 & 1 & 0 & 2 & 1 & 0 & 1 & 0 & 0 & & 1 & 1 & 1 & & & 0 & 0 & 0 & 0 \\
\hline 131 & 1 & 1 & 2 & 2 & 0 & 1 & 0 & 2 & 3 & 0 & 1 & 0 & 0 & & 1 & 2 & 2 & & & 0 & 0 & 0 & 1 \\
\hline 32 & 0 & 0 & 0 & 1 & 0 & 1 & 0 & 0 & 0 & 0 & 1 & 0 & 0 & & 0 & 1 & 0 & & & 0 & 0 & 0 & 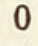 \\
\hline 133 & 0 & 1 & 0 & 2 & 0 & 2 & 0 & 0 & 1 & 0 & 1 & 0 & 0 & & 1 & 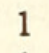 & & & & 0 & 0 & 1 & 1 \\
\hline 134 & 0 & 1 & 0 & 1 & 0 & 1 & 0 & 1 & 2 & 0 & 1 & 0 & 0 & & 0 & 1 & & & & 0 & 0 & 0 & 0 \\
\hline 135 & 0 & 0 & 0 & 2 & 0 & 1 & 0 & 0 & 1 & 0 & 1 & 0 & 0 & & 0 & 1 & 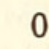 & & & 0 & 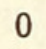 & 1 & 0 \\
\hline 136 & 0 & 1 & 1 & 2 & 0 & 2 & 0 & 2 & 2 & 0 & 1 & 0 & 0 & & 1 & 3 & & & & 0 & 0 & 0 & 0 \\
\hline 137 & 0 & 0 & 1 & 2 & 0 & 1 & 0 & 1 & 2 & 0 & 1 & 0 & 0 & & 1 & 0 & 0 & & & 0 & 0 & 0 & 0 \\
\hline 138 & 0 & 1 & 2 & 1 & 0 & 1 & 0 & 1 & 1 & 0 & 2 & 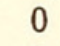 & 0 & & 0 & 2 & & & & 0 & 0 & 0 & 1 \\
\hline 139 & 0 & 0 & 0 & 1 & 0 & 1 & 0 & 2 & 1 & 0 & 1 & 0 & 0 & & 1 & 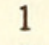 & & & & 0 & 0 & 0 & 0 \\
\hline 140 & 0 & 0 & 0 & 1 & 0 & 1 & 0 & 1 & 2 & 0 & 1 & 0 & 0 & & 0 & 0 & 1 & & & 0 & 0 & 0 & 0 \\
\hline 141 & 1 & 1 & 1 & 1 & 0 & 1 & 0 & 1 & 2 & 0 & 1 & 0 & 0 & & 1 & 1 & 1 & & & 0 & 0 & 0 & 2 \\
\hline 142 & 1 & 1 & 1 & 2 & 0 & 2 & 0 & 2 & 2 & 0 & 2 & 0 & 0 & & 1 & 2 & 1 & & & 0 & 0 & 0 & 1 \\
\hline 143 & 1 & 1 & 1 & 2 & 0 & 2 & 0 & 1 & 3 & 0 & 1 & 0 & 0 & & 1 & 1 & 1 & & 0 & 0 & 0 & 0 & 1 \\
\hline 144 & 1 & 1 & 1 & 1 & 0 & 2 & 0 & 1 & 1 & 0 & 1 & 0 & 0 & & 1 & 3 & 1 & & 0 & 0 & 0 & 0 & 1 \\
\hline 145 & 1 & 1 & 1 & 3 & 0 & 3 & 0 & 2 & 3 & 0 & 2 & 0 & 0 & & 1 & 2 & 1 & & 0 & 0 & 0 & 1 & 1 \\
\hline 146 & 0 & 1 & 0 & 2 & 0 & 2 & 0 & 1 & 1 & 0 & 1 & 0 & 0 & & 0 & 2 & 0 & & 0 & 0 & 0 & 1 & 1 \\
\hline 147 & 1 & 1 & 1 & 1 & 0 & 1 & 0 & 1 & 2 & 0 & 1 & 0 & 0 & 0 & 0 & 3 & 0 & & 0 & 0 & 0 & 0 & 1 \\
\hline 148 & 0 & 0 & 1 & 2 & 0 & 2 & 0 & 3 & 3 & 0 & 2 & 0 & 0 & & 1 & 1 & 0 & & 0 & 0 & 0 & 0 & 0 \\
\hline 149 & 1 & 0 & 1 & 3 & 0 & 0 & 0 & 2 & 4 & 0 & 2 & 0 & 0 & & 0 & 1 & 1 & & 0 & 0 & 0 & 0 & 0 \\
\hline 150 & 0 & 0 & 0 & 2 & 0 & 1 & 0 & 2 & 3 & 0 & 1 & 0 & 0 & 0 & 0 & 0 & 1 & & 0 & 0 & 0 & 0 & 0 \\
\hline 151 & 1 & 0 & 0 & 0 & 0 & 0 & 0 & 1 & 1 & 0 & 2 & 0 & 0 & & 0 & 0 & 0 & & 0 & 0 & 0 & 0 & 0 \\
\hline 152 & 1 & 1 & 2 & 3 & 0 & 2 & 1 & 3 & 4 & 0 & 3 & 0 & 0 & & 1 & 1 & 1 & & 1 & 0 & 0 & 0 & 0 \\
\hline 153 & 1 & 1 & 0 & 1 & 0 & 0 & 0 & 1 & 3 & 0 & 1 & 0 & 0 & & 0 & 0 & 0 & & 0 & 0 & 0 & 0 & 0 \\
\hline 154 & 1 & 1 & 2 & 3 & 0 & 2 & 1 & 3 & 4 & 0 & 3 & 0 & $c$ & 0 & 1 & 3 & 1 & & 1 & 0 & 0 & 0 & 0 \\
\hline 155 & 1 & 0 & 1 & 0 & 0 & 1 & 0 & 2 & 3 & 0 & 1 & 0 & 0 & 0 & 1 & 1 & 1 & & 0 & 0 & 0 & 0 & 1 \\
\hline 156 & 0 & 0 & 1 & 1 & 0 & 1 & 0 & 1 & 3 & 0 & 2 & 0 & 0 & & i & 1 & 1 & & 0 & 0 & 0 & 0 & 1 \\
\hline 157 & 0 & 0 & 1 & 3 & 0 & 0 & 0 & 2 & 4 & 0 & 2 & 1 & 0 & 0 & 0 & 1 & 1 & & 1 & 0 & 0 & 1 & 0 \\
\hline 158 & 0 & 0 & 1 & 1 & 0 & 2 & 0 & 2 & 4 & 0 & 1 & 1 & 0 & 0 & 1 & 0 & 1 & & 0 & 0 & 0 & 0 & 0 \\
\hline 15 & 0 & 0 & & & 0 & 2 & & 2 & J & 0 & & 0 & 0 & & & 2 & & & & & & & \\
\hline
\end{tabular}




\section{APPENDIX 1. Continued}

Site Bla Sym Aca Echinoph Oxy Euph Parac Poly Heteroc Cary Bal Rhiz Den Tub Tur Heterop Tubip Mille Stylas Unid G S

\begin{tabular}{|c|c|c|c|c|c|c|c|c|c|c|c|c|c|c|c|c|c|c|c|c|c|c|}
\hline 106 & 1 & 0 & 0 & 0 & 1 & 0 & 0 & 0 & 0 & 0 & 0 & 0 & 0 & 1 & 0 & 0 & 0 & 0 & 0 & 0 & 20 & 24 \\
\hline 107 & 0 & 0 & 0 & 0 & 0 & 0 & 1 & 1 & 0 & 0 & 1 & 1 & 1 & 0 & 0 & 0 & 0 & 0 & 0 & 0 & 10 & 10 \\
\hline 108 & 0 & 0 & 1 & 1 & 1 & 0 & 0 & 0 & 0 & 0 & 1 & 0 & 0 & 1 & 2 & 1 & 0 & 0 & 0 & 0 & 19 & 23 \\
\hline 109 & 0 & 1 & 1 & 1 & 0 & 0 & 0 & 0 & 0 & 0 & 0 & 0 & 0 & 0 & 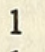 & & & 0 & 0 & 0 & 18 & \\
\hline 10 & 0 & 1 & 1 & 1 & 0 & 0 & 0 & 0 & 0 & 0 & 0 & 0 & 0 & 0 & , & & & 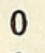 & 0 & 0 & 22 & 29 \\
\hline 11 & 0 & 1 & 1 & 1 & 0 & 0 & 0 & 0 & 0 & 0 & 0 & 0 & 0 & 0 & 1 & 0 & & 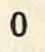 & 0 & 0 & 17 & 4 \\
\hline 12 & 0 & 1 & 1 & 1 & 0 & 0 & 0 & 0 & 0 & 0 & 0 & 0 & 0 & 0 & 1 & 0 & & . & 0 & 0 & 13 & 3 \\
\hline 13 & 1 & 1 & 1 & 1 & 1 & 0 & 0 & 0 & 0 & 0 & 0 & 0 & 0 & 1 & 1 & 0 & & 0 & 0 & 0 & 22 & \\
\hline 14 & 0 & 1 & 1 & 1 & 0 & 0 & 0 & 0 & 0 & 0 & 0 & 0 & 0 & 0 & 1 & 0 & & 0 & 0 & ) & 15 & 21 \\
\hline 15 & 0 & 0 & 0 & 0 & 0 & 0 & 0 & 0 & 0 & 0 & 0 & 0 & 0 & 0 & 0 & 0 & & 0 & & ) & 8 & \\
\hline 0 & 1 & 1 & 1 & 1 & 1 & 0 & 0 & 0 & 0 & 0 & 0 & 0 & 0 & 2 & & & & 0 & & 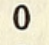 & 25 & \\
\hline 17 & 1 & 1 & 0 & 0 & 1 & 0 & 0 & 0 & 0 & 0 & 0 & 0 & 0 & 0 & - & 0 & & 0 & 0 & 0 & 12 & 2 \\
\hline 18 & 0 & 1 & 1 & 1 & 1 & 0 & 0 & 0 & 0 & 0 & 0 & 0 & 0 & 0 & 2 & 0 & & 0 & 0 & 0 & 25 & 3 \\
\hline 19 & 0 & 1 & 1 & 1 & 0 & 0 & 0 & 0 & 0 & 0 & 0 & 0 & 0 & 0 & 2 & 0 & & 0 & 0 & 0 & 23 & \\
\hline 0 & 0 & 1 & 1 & 1 & 1 & 0 & 0 & 0 & 0 & 0 & 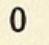 & 0 & 0 & 0 & ? & ( & & 0 & 0 & 0 & 25 & 0 \\
\hline 121 & 1 & 1 & 1 & 1 & 1 & 0 & 0 & 0 & 0 & 0 & 0 & 0 & 0 & 0 & 1 & 0 & & 0 & 0 & 0 & 30 & 0 \\
\hline 122 & 0 & 1 & 1 & 1 & 0 & 0 & 0 & 0 & 0 & 0 & 0 & 0 & 0 & 0 & 2 & 0 & & 0 & & ) & 21 & 7 \\
\hline 23 & 0 & 0 & 1 & 0 & 0 & 0 & 0 & 0 & 0 & 0 & 0 & 0 & 0 & 0 & 1 & 0 & J & $c$ & 0 & 0 & 0 & \\
\hline 24 & 1 & 1 & 1 & 1 & 1 & 0 & 0 & 0 & 0 & 0 & 0 & 0 & 0 & 0 & 1 & 0 & & 0 & 0 & 0 & 24 & 27 \\
\hline 25 & 0 & 1 & 1 & 0 & 0 & 0 & 0 & 0 & 0 & 0 & 0 & 0 & 0 & 0 & 1 & 0 & & $c$ & 0 & 0 & 16 & 17 \\
\hline 26 & 0 & 1 & 1 & 1 & 1 & 0 & 0 & 0 & 0 & 0 & 0 & 0 & 0 & 0 & 1 & 0 & 0 & 0 & 0 & 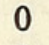 & 21 & D \\
\hline I & 1 & 1 & 2 & 1 & 1 & 0 & 1 & 0 & 0 & 0 & 0 & 0 & 1 & 1 & 2 & 0 & 0 & c & 0 & 0 & 34 & 3 \\
\hline 128 & 1 & 1 & 1 & 1 & 0 & 0 & 0 & 0 & 0 & 0 & 0 & 0 & 0 & 0 & 1 & 0 & 0 & 0 & 0 & 0 & 24 & 31 \\
\hline 129 & 0 & 1 & 1 & 1 & 0 & 0 & 0 & 0 & 0 & 0 & 0 & 0 & 0 & 0 & 1 & 0 & J & $c$ & 0 & 0 & 22 & \\
\hline 130 & 0 & 1 & 1 & 1 & 0 & 0 & 0 & 0 & 0 & 0 & 0 & 0 & 0 & 0 & 1 & 0 & 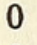 & ( & 0 & 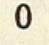 & 22 & 24 \\
\hline 31 & 0 & 1 & 2 & 1 & 1 & 0 & 0 & 0 & 0 & 0 & 0 & 0 & 0 & 0 & 2 & 0 & J & ( & 0 & 0 & 27 & 41 \\
\hline 132 & 0 & 1 & 0 & 0 & 0 & 0 & 0 & 0 & 0 & 0 & 0 & 0 & 0 & 0 & 1 & $c$ & 0 & $c$ & 0 & J & 12 & 12 \\
\hline 133 & 0 & 1 & 1 & 1 & 0 & 0 & 0 & 0 & 0 & 0 & 0 & 0 & 0 & 0 & 1 & ( & 0 & & 0 & 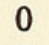 & 22 & 20 \\
\hline 134 & 0 & 1 & 0 & 0 & 0 & 0 & 0 & 0 & 0 & 0 & 0 & 0 & 0 & 0 & 0 & 0 & 0 & c & 0 & 0 & 16 & 20 \\
\hline 135 & 0 & 1 & 1 & 0 & 0 & 0 & 0 & 0 & 0 & 0 & 0 & 0 & 1 & 0 & 1 & 0 & 0 & 0 & 0 & 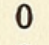 & 19 & 20 \\
\hline 136 & 1 & 1 & 1 & 1 & 1 & 0 & 0 & 0 & 0 & 0 & 0 & 0 & 1 & 1 & 0 & & 0 & c & 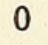 & 0 & 26 & 36 \\
\hline 137 & 0 & 1 & 1 & 0 & 0 & 0 & 0 & 0 & 0 & 0 & 0 & 0 & 0 & 0 & 1 & 0 & 0 & & 0 & ) & 17 & 20 \\
\hline 138 & 1 & 1 & 1 & 0 & 1 & 0 & 0 & 0 & 0 & 0 & 0 & 0 & 1 & 0 & 2 & 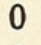 & 0 & 0 & 0 & 0 & 25 & 31 \\
\hline 139 & 0 & 1 & 1 & 1 & 0 & 0 & 0 & 0 & 0 & 0 & 0 & 0 & 0 & 0 & 1 & 0 & 0 & 0 & 0 & 0 & 16 & 17 \\
\hline 14 & 0 & 1 & 1 & 0 & 0 & 0 & 0 & 0 & 0 & 0 & 0 & 0 & 0 & 0 & 0 & 0 & 0 & 0 & 0 & 0 & 14 & 16 \\
\hline 141 & 1 & 1 & 1 & 1 & 0 & 0 & 0 & 0 & 0 & 0 & 0 & 0 & 0 & 0 & 2 & 0 & 0 & 0 & 0 & 0 & 25 & 33 \\
\hline 142 & 1 & 1 & 1 & 0 & 0 & 0 & 0 & 0 & 0 & 0 & 0 & 0 & 0 & 0 & 3 & 0 & 0 & 0 & 0 & 0 & 19 & 29 \\
\hline 143 & 1 & 1 & 1 & 1 & 0 & 0 & 0 & 0 & 0 & 0 & 0 & 0 & 0 & 0 & 3 & 0 & 0 & 0 & 0 & 0 & 24 & 4 \\
\hline 144 & 1 & 1 & 1 & 1 & 1 & 0 & 0 & 0 & 0 & 0 & 0 & 0 & 0 & 1 & 3 & 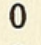 & 0 & 0 & 0 & 0 & 27 & 33 \\
\hline 145 & 1 & 1 & 2 & 1 & 0 & 0 & 0 & 0 & 0 & 0 & 0 & 0 & 0 & 0 & 3 & 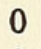 & 0 & 0 & 0 & 0 & 27 & 44 \\
\hline 146 & 0 & 0 & 1 & 0 & 0 & 0 & 0 & 0 & 0 & 0 & 0 & 0 & 0 & 0 & 1 & 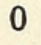 & 0 & 0 & 0 & 0 & 19 & 23 \\
\hline 147 & 0 & 1 & 1 & 0 & 0 & 0 & 0 & 0 & 0 & 0 & 0 & 0 & 0 & 0 & 3 & 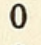 & 0 & 0 & 0 & 0 & 20 & 28 \\
\hline 148 & 0 & 0 & 2 & 1 & 0 & 0 & 0 & 0 & 0 & 0 & 0 & 0 & 0 & 0 & 3 & 0 & 0 & 0 & 0 & 0 & 18 & 32 \\
\hline 149 & 0 & 0 & 0 & 1 & 0 & 0 & 0 & 0 & 0 & 0 & 0 & 0 & 0 & 0 & 0 & 0 & 0 & 0 & 0 & 0 & 12 & 21 \\
\hline 150 & 0 & 0 & 0 & 0 & 0 & 0 & 0 & 0 & 0 & 0 & 0 & 0 & 0 & 0 & 1 & 0 & 0 & 0 & 0 & 0 & 11 & 7 \\
\hline 151 & 0 & 0 & 0 & 0 & 0 & 0 & 0 & 0 & 0 & 0 & 0 & 0 & 0 & 0 & 1 & 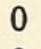 & 0 & 0 & 0 & 0 & 9 & 11 \\
\hline 152 & 0 & 0 & 2 & 0 & 0 & 0 & 0 & 0 & 0 & 0 & 0 & 0 & 0 & 0 & 3 & 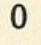 & 0 & 0 & 0 & 0 & 20 & 35 \\
\hline 153 & 0 & 0 & 0 & 0 & 0 & 0 & 0 & 0 & 0 & 0 & 0 & 0 & 0 & 0 & 0 & 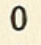 & 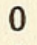 & 0 & 0 & 0 & 8 & 10 \\
\hline 154 & 0 & 0 & 2 & 1 & 0 & 0 & 0 & 0 & 1 & 0 & 0 & 0 & 0 & 0 & 3 & 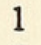 & 0 & 0 & 0 & 0 & 27 & 47 \\
\hline 55 & 0 & 0 & 2 & 1 & 1 & 0 & 0 & 0 & 0 & 0 & 0 & 0 & 0 & 0 & 1 & 0 & 0 & 0 & 0 & 0 & 21 & 28 \\
\hline 156 & 0 & 0 & 1 & 0 & 1 & 0 & 0 & 0 & 0 & 0 & 0 & 0 & 1 & 1 & 1 & 0 & 0 & 0 & 0 & 0 & 23 & 10 \\
\hline 157 & 1 & 1 & 1 & 1 & 1 & 0 & 0 & 0 & 0 & 0 & 0 & 0 & 0 & 0 & 2 & 0 & U & ) & 0 & 0 & 25 & 37 \\
\hline 158 & 0 & 0 & 1 & 1 & 1 & 0 & 0 & 0 & 0 & 0 & 0 & 0 & 0 & 0 & 1 & 0 & & 0 & 0 & 0 & 20 & \\
\hline 159 & n & 1 & 1 & 1 & 1 & 0 & 0 & 0 & 0 & 0 & 0 & 0 & 0 & 0 & 2 & 0 & 0 & 0 & 0 & 0 & 22 & \\
\hline
\end{tabular}


APPENDIX 1. Continued

Site Site Name

$\begin{array}{ll}\text { 160 Wadi Aingalf E } & \text { BD746773 } \\ \text { 161 Raaha E } & \text { BD664749 } \\ \text { 162 Raaha W } & \text { BD663747 } \\ \text { 163 Raaha } & \text { BD654743 } \\ \text { 164 Jazirat Hino W } & \text { BD594755 } \\ \text { 165 Ra's Marbat } & \text { BD530786 } \\ \text { 166 Ra's Raysut } & \text { ZU208749 } \\ \text { 167 Rock Stack } & \text { ZU190740 } \\ \text { 168 Ra's Hamar } & \text { ZU140710 } \\ \text { 169 Fizayih } & \text { YU892631-888625 } \\ \text { 170 Ghubbat Fizayih W } & \text { YU846574-850584 } \\ \text { 171 Millepora Bay } & \text { YU710544 } \\ \text { 172 Ma'alqawt } & \text { YU700546-693548 } \\ \text { 173 Kharfot } & \text { YU488508 } \\ \text { 174 Hallaniyah (Gnl) } & \text { CE960360 } \\ \text { 175 Hallaniyah NW } & \text { CE900364 } \\ \text { 176 Hallaniyah SE } & \text { DE032355 } \\ \text { 177 Hallaniyah S2 } & \text { CE929338 } \\ \text { 178 Hallaniyah SW } & \text { CE911342 } \\ \text { 179 Hallaniyah SW } & \text { CE911342 } \\ \text { 180 As Sawda (Gnl) } & \text { CE780350 } \\ \text { 181 As Sawda NE } & \text { CE808346 } \\ \text { 182 As Sawda E } & \text { CE808334 } \\ \text { 183 As Sawda S } & \text { CE781336 } \\ \text { 184 As Sawda NW } & \text { CE760350 } \\ \text { 185 Dhofar (Gnl) } & \end{array}$

Psam Styloc Poc Stylop Ser Mad Ast Acr Monti Pav Leptos Gar Sid

$\begin{array}{lllllllllllll}1 & 0 & 0 & 1 & 0 & 0 & 0 & 2 & 2 & 2 & 1 & 1 & 0 \\ 0 & 0 & 0 & 1 & 0 & 0 & 0 & 4 & 2 & 2 & 0 & 1 & 0 \\ 0 & 0 & 0 & 1 & 0 & 0 & 1 & 2 & 2 & 2 & 1 & 0 & 0 \\ 0 & 0 & 0 & 1 & 0 & 0 & 0 & 2 & 2 & 2 & 0 & 1 & 0 \\ 0 & 0 & 1 & 1 & 0 & 0 & 0 & 1 & 1 & 0 & 0 & 0 & 0 \\ 2 & 1 & 0 & 1 & 0 & 1 & 0 & 1 & 2 & 1 & 2 & 1 & 0 \\ 0 & 0 & 0 & 1 & 0 & 0 & 0 & 0 & 0 & 0 & 0 & 1 & 0 \\ 0 & 0 & 0 & 1 & 0 & 0 & 0 & 1 & 1 & 1 & 0 & 1 & 0 \\ 0 & 0 & 0 & 1 & 0 & 0 & 0 & 1 & 1 & 0 & 0 & 1 & 0 \\ 0 & 0 & 0 & 1 & 0 & 0 & 0 & 1 & 1 & 0 & 0 & 1 & 0 \\ 0 & 0 & 0 & 0 & 0 & 0 & 0 & 0 & 0 & 0 & 0 & 0 & 0 \\ 0 & 0 & 0 & 1 & 0 & 0 & 0 & 3 & 3 & 2 & 0 & 1 & 0 \\ 0 & 0 & 0 & 1 & 0 & 0 & 0 & 1 & 1 & 0 & 0 & 0 & 0 \\ 0 & 0 & 0 & 0 & 0 & 0 & 0 & 0 & 1 & 0 & 0 & 1 & 1 \\ 0 & 0 & 1 & 1 & 0 & 1 & 0 & 3 & 3 & 3 & 1 & 0 & 1 \\ 0 & 0 & 1 & 1 & 0 & 0 & 0 & 2 & 3 & 3 & 0 & 0 & 1 \\ 0 & 0 & 0 & 1 & 0 & 0 & 0 & 3 & 1 & 0 & 0 & 0 & 0 \\ 0 & 0 & 0 & 1 & 0 & 1 & 0 & 3 & 3 & 2 & 1 & 0 & 0 \\ 0 & 0 & 0 & 1 & 0 & 0 & 0 & 2 & 2 & 0 & 0 & 0 & 0 \\ 0 & 0 & 0 & 1 & 0 & 0 & 0 & 2 & 1 & 1 & 0 & 0 & 0 \\ 1 & 0 & 0 & 2 & 0 & 0 & 1 & 3 & 3 & 3 & 1 & 0 & 0 \\ 0 & 0 & 0 & 1 & 0 & 0 & 1 & 2 & 2 & 0 & 1 & 0 & 0 \\ 1 & 0 & 0 & 2 & 0 & 0 & 0 & 3 & 3 & 2 & 1 & 0 & 0 \\ 0 & 0 & 0 & 2 & 0 & 0 & 1 & 3 & 3 & 3 & 0 & 0 & 0 \\ 0 & 0 & 0 & 1 & 0 & 0 & 0 & 3 & 1 & 0 & 0 & 0 & 0 \\ 2 & 1 & 1 & 1 & 1 & 1 & 1 & 4 & 3 & 3 & 2 & 1 & 1\end{array}$


APPENDIX 1. Continued

Site Pseud Ano Cos Por P(Syn) Gonio Alv Favia Favit Gonia Pla Leptor Monta Ples Lepta Cyph Echin Cul Phy Gal Par Hyd

$\begin{array}{lllllllllllllllllllllll}160 & 0 & 0 & 1 & 0 & 0 & 2 & 0 & 2 & 5 & 0 & 2 & 0 & 0 & 1 & 2 & 1 & 1 & 0 & 0 & 0 & 0 & 2 \\ 161 & 0 & 0 & 1 & 1 & 0 & 1 & 0 & 1 & 3 & 0 & 2 & 0 & 0 & 0 & 1 & 1 & 1 & 0 & 0 & 1 & 0 & 1 \\ 162 & 0 & 0 & 1 & 3 & 0 & 1 & 0 & 1 & 3 & 0 & 2 & 0 & 0 & 0 & 0 & 1 & 1 & 0 & 0 & 1 & 0 & 1 \\ 163 & 1 & 0 & 1 & 1 & 0 & 1 & 0 & 1 & 3 & 0 & 2 & 0 & 0 & 1 & 1 & 1 & 1 & 0 & 0 & 0 & 0 & 2 \\ 164 & 0 & 0 & 1 & 3 & 0 & 1 & 0 & 2 & 2 & 0 & 2 & 0 & 0 & 1 & 0 & 1 & 1 & 0 & 0 & 0 & 0 & 2 \\ 165 & 1 & 0 & 1 & 0 & 0 & 1 & 0 & 2 & 3 & 0 & 1 & 0 & 0 & 1 & 1 & 1 & 0 & 0 & 0 & 0 & 0 & 0 \\ 166 & 0 & 0 & 2 & 0 & 0 & 1 & 0 & 1 & 2 & 0 & 0 & 0 & 0 & 0 & 1 & 0 & 0 & 0 & 0 & 0 & 0 & 0 \\ 167 & 1 & 0 & 1 & 0 & 0 & 0 & 0 & 1 & 1 & 0 & 1 & 0 & 0 & 0 & 2 & 1 & 0 & 0 & 0 & 0 & 0 & 2 \\ 168 & 1 & 0 & 1 & 1 & 0 & 1 & 0 & 1 & 2 & 0 & 1 & 0 & 0 & 1 & 1 & 1 & 0 & 0 & 0 & 0 & 1 & 0 \\ 169 & 1 & 0 & 0 & 3 & 0 & 1 & 0 & 0 & 1 & 0 & 1 & 0 & 0 & 0 & 0 & 0 & 0 & 0 & 0 & 0 & 0 & 0 \\ 170 & 0 & 0 & 0 & 0 & 0 & 0 & 0 & 0 & 0 & 0 & 0 & 0 & 0 & 0 & 0 & 0 & 0 & 0 & 0 & 0 & 0 & 0 \\ 171 & 0 & 1 & 1 & 1 & 0 & 1 & 0 & 1 & 3 & 0 & 2 & 0 & 0 & 1 & 0 & 1 & 1 & 0 & 0 & 0 & 0 & 2 \\ 172 & 0 & 0 & 0 & 0 & 0 & 0 & 0 & 0 & 0 & 0 & 0 & 0 & 0 & 0 & 0 & 0 & 0 & 0 & 0 & 0 & 0 & 0 \\ 173 & 0 & 0 & 1 & 0 & 0 & 1 & 0 & 0 & 1 & 0 & 1 & 0 & 0 & 1 & 1 & 0 & 0 & 0 & 0 & 0 & 1 & 0 \\ 174 & 1 & 1 & 1 & 3 & 0 & 2 & 1 & 3 & 3 & 1 & 2 & 1 & 0 & 1 & 2 & 3 & 1 & 0 & 0 & 1 & 1 & 2 \\ 175 & 1 & 1 & 1 & 3 & 0 & 2 & 0 & 3 & 2 & 0 & 1 & 1 & 0 & 1 & 1 & 1 & 1 & 0 & 0 & 1 & 1 & 2 \\ 176 & 0 & 1 & 0 & 3 & 0 & 1 & 1 & 1 & 3 & 0 & 1 & 1 & 0 & 0 & 1 & 1 & 0 & 0 & 0 & 0 & 0 & 1 \\ 177 & 1 & 0 & 1 & 3 & 0 & 2 & 0 & 3 & 2 & 1 & 1 & 1 & 0 & 1 & 2 & 3 & 1 & 0 & 0 & 1 & 0 & 1 \\ 178 & 0 & 0 & 0 & 3 & 0 & 1 & 0 & 1 & 2 & 0 & 1 & 1 & 0 & 0 & 1 & 0 & 0 & 0 & 0 & 1 & 0 & 0 \\ 179 & 1 & 0 & 1 & 3 & 0 & 2 & 1 & 2 & 2 & 0 & 2 & 1 & 0 & 0 & 2 & 1 & 1 & 0 & 0 & 1 & 1 & 2 \\ 180 & 1 & 1 & 1 & 4 & 0 & 2 & 1 & 4 & 3 & 1 & 2 & 1 & 0 & 1 & 1 & 2 & 1 & 0 & 0 & 1 & 1 & 2 \\ 181 & 0 & 1 & 1 & 2 & 0 & 2 & 1 & 2 & 2 & 0 & 1 & 1 & 0 & 1 & 1 & 0 & 1 & 0 & 0 & 1 & 1 & 2 \\ 182 & 0 & 1 & 1 & 2 & 0 & 1 & 0 & 3 & 2 & 0 & 1 & 1 & 0 & 0 & 1 & 2 & 1 & 0 & 0 & 1 & 0 & 2 \\ 183 & 1 & 0 & 1 & 4 & 0 & 1 & 0 & 4 & 3 & 1 & 2 & 1 & 1 & 1 & 0 & 1 & 1 & 0 & 0 & 1 & 0 & 2 \\ 184 & 0 & 0 & 0 & 1 & 0 & 0 & 0 & 1 & 2 & 0 & 2 & 0 & 0 & 0 & 1 & 0 & 0 & 0 & 0 & 0 & 0 & 1 \\ 185 & 1 & 1 & 2 & 4 & 0 & 2 & 2 & 4 & 5 & 1 & 2 & 1 & 1 & 1 & 2 & 2 & 1 & 1 & 1 & 1 & 1 & 2\end{array}$


APPENDIX 1. Continued

Site Bla Sym Aca Echinoph Oxy Euph Parac Poly Heteroc Cary Bal Rhiz Den Tub Tur Heterop Tubip Mille Stylas Unid G S

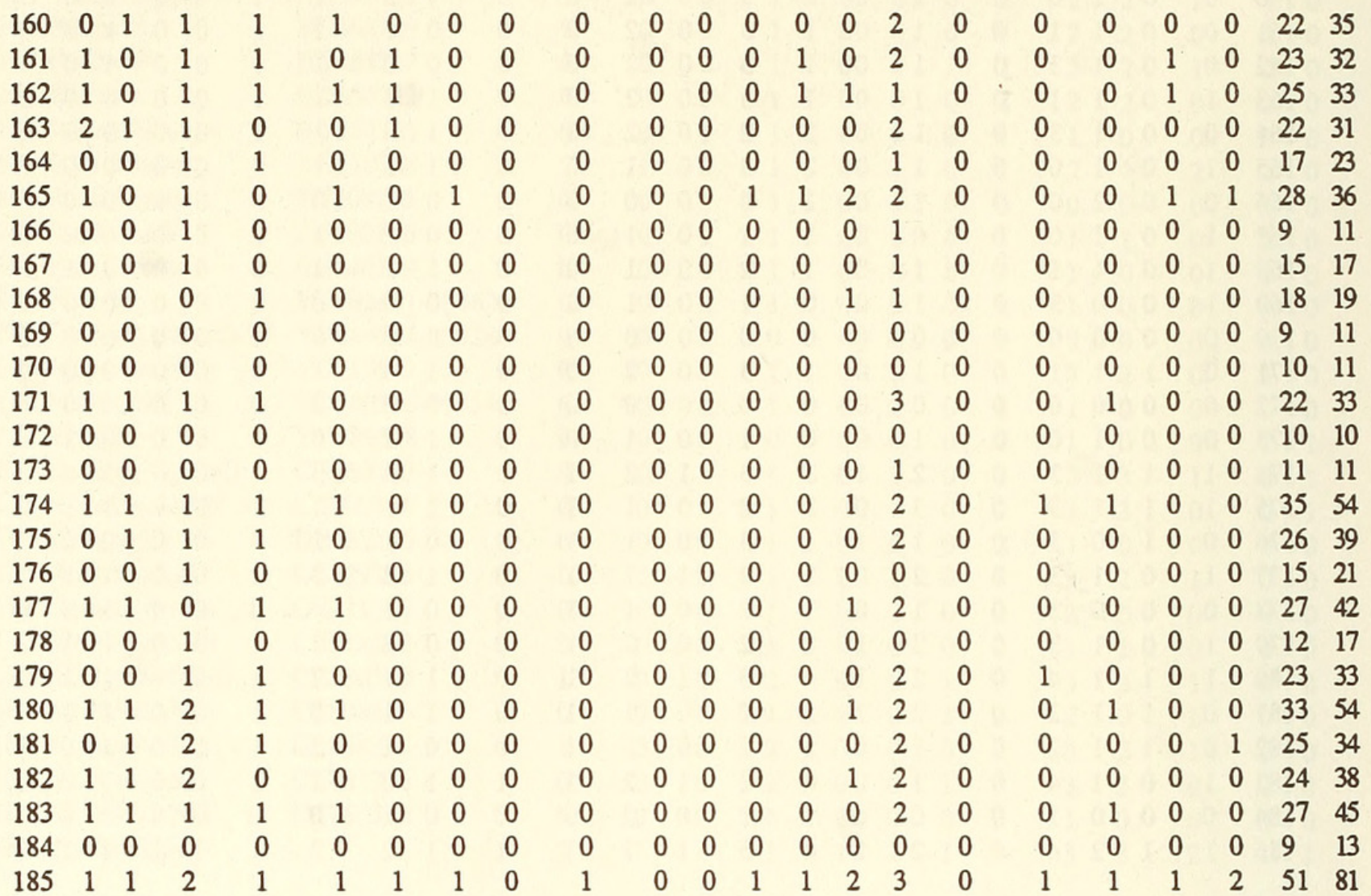




\section{APPENDIX 2. DESCRIPTIONS OF CORAL COMMUNITIES IN OMAN}

This appendix lists summary descriptions for 246 coral communities studied during the course of the coastal zone management project. It derives from a general environmental classification database. The grid coordinates on the National Survey Authority Map Series K6611 (1:100,000) are listed after the name of each site. A type A coral community is one with true framework development. A type B community is one where corals are settled directly on base rock, sometimes obscuring it almost entirely, but where there is no reef framework. A type $\mathbf{C}$ community has corals growing mixed with or bordered by dense seasonal phaeophyte (brown algae) communities. Where a coral list has been completed, the site number corresponding to Appendix 1 is listed after the community type classification.

Ra's al Jirri - Ra's Shaykh Mas'ud (DQ190015-220040)

Coral Community Type: A, B (site number 001)

Description: The coast is characterized by cliff of varying height falling to a base of tumbled rock boulders on sand at depths of $1-5 \mathrm{~m}$. Shallower rocks are encrusted by pale brown zoanthid (Palythoa sp.), with scattered corals deeper surrounded by algal turf. Some coves have abundant $P$. damicornis. There are numerous juvenile fishes, especially chaetodons, angelfishes, sergeant majors, and some $P$. versicolor.

Threats: gill net, ropes, lines, anchors, tarballs, litter ashore; fabric, litter in coral; use by Iranians results in direct damage to coral from anchors, ballast bags of sand dumped overboard, in addition to litter on shore and underwater.

Cove 1, Ra's Shaykh Mas'ud (DQ198022)

Coral Community Type: B (site number 002)

Threats: lines in coral.

\section{Khawr Idah South (DQ200026)}

Coral Community Type: A, B (site number 003)

Description: coral growth well developed south side where small patch of Porites fused into a solid flat-topped framework $1.3 \mathrm{~m}$ thick. Elsewhere coral generally in dispersed patches formed of Porites with large tabular Acropora. Towards centre of bay large Porites ( $2-3 \mathrm{~m}$ diameter to $4 \mathrm{~m}$ maximum) fused to form substantial spurs. North side has small scattered corals on rock.

Threats: ropes, fabric in coral, tarballs.

Ra's Shaykh Mas'ud West (DQ214041)

Coral Community Type: B (site number 004)

Description: west side has good coral growth with $1-2 \mathrm{~m}$ diameter Porites and large tabular Acropora. There is a dense ( $99 \%$ cover) patch of $P$. damicornis.

Threats: gill net in coral, tarballs, litter, flotsam; corals damaged by anchors and numerous sand-filled ballast bags of Iranian small boat traders. 
Qabr Shaykh Mas'ud (DQ217042)

Coral Community Type: B

Description: attractive coral growth on rocks bordering and in middle of bay.

Threats: tarballs, severe damage underwater caused by ballast bags, anchors, ropes, trash of Iranian small boat traders.

Ra's Salti Ali (DP237994)

Coral Community Type: B (site number 005)

Description: shallow sandy bay bordered by boulders, large undercut rock blocks, becoming larger toward point, scattered corals.

Threats: severe damage by ropes, gill nets, anchor blocks; much smashed, dead coral, litter underwater, tarballs.

Ra's Shakhs (DP280997-284998)

Coral Community Type: A, B (site number 006)

Description: narrow, low reef parallel shore, maximum framework of rubble banks 0.5-1m, covered by $P$. damicornis, bushy and tabular Acropora; cover bushy/tabular Acropora $10-60 \%$ to $95 \%$ for $P$. damicornis; much coral on beach.

Threats: gill nets, ropes, anchor blocks in and breaking coral; severe damage from monofilament net that had entangled coral, fishes and many lobsters and large crabs.

Khawr ash Shamm (DP340980)

Coral Community Type: A, B (site number 007)

Description: sheltered fjordlike bay; much shore fringed by coral; some well-developed Porites framework reefs; luxuriant Acropora thickets with fragile extended branches; patches $P$. cactus framework reefs e.g. DP324966; coral growth appears better developed central and inner bay than outer - many more patches framework reef and luxuriant Acropora.

Threats: Acropora smashed by fish traps, anchors; coral hauled up in gill nets; gill nets, fish traps in coral; Platygyra white patch disease severe inner bay.

Khawr ash Shamm (Jazirat al Maqlab) (DP344974)

Coral Community Type: A, B (site number 008)

Description: almost entirely encircled by Porites framework reef with luxuriant thickets Acropora below, patches $P$. cactus and $P$. ?decussata; Porites reef best developed east side, $2-4 \mathrm{~m}$ framework, solid.

Threats: Acropora smashed by fish traps, anchors.

Khawr ash Shamm (Maksar) (DP395980)

Coral Community Type: B (site number 009)

Description: scattered coral 50-85\% cover, predominantly Platygyra, Cyphastrea, $P$. ?decussata.

Threats: cans underwater, Platygyra frequently have large white patches leading from yellow central patch of fine alga through white band to thin tissue bordering healthy area - no black band or other sign of infection; much dead coral. 
Khawr ash Shamm (Jazirat Sibi) (DQ394004)

Coral Community Type: A, B (site number 010)

Description: shallow sandy shelves east and west covered by coral; east side Porites leading to mix Acropora thicket, Platygyra, and $P$. ?decussata to slope with $P$. ?decussata reef framework $>1 \mathrm{~m}$ thick; west side mixed Acropora, scattered $P$. ?decussata, Platygyra to slope with Acropora and $P$. ?decussata; rest of island rocky steep slope with predominantly Platygyra in shallows and steep slopes, $P$. ?decussata other slopes.

Threats: much dead coral; Platygyra white patch disease.

Ghassah (DQ320026)

Coral Community Type: A, B (site number 011)

Description: bay bordered by rock with scattered coral, patches tabular and bushy Acropora; talus banks scattered through bay with bushy Acropora; northeast corner DQ321035 fused flat-topped Porites reef, framework $0.5-1.5 \mathrm{~m}$ thick, bordered by sand with scattered Porites $<3 \mathrm{~m}$ diameter and tabular Acropora.

Threats: much flotsam, old small tarballs, much dead coral.

Khawr Ghubb Ali (DQ350060)

Coral Community Type: A, B (site number 012)

Description: bay fringed by narrow border coral, primarily bushy and tabular Acropora, Porites, $P$. damicornis, Platygyra, $P$. cactus - each predominant at different sites; talus banks, patches bushy Acropora extend well offshore in places of ca. $6 \mathrm{~m}$ depth; massive corals hollowed out; southwest coves have fused Porites reefs with $1.5 \mathrm{~m}$ framework, cover varies $70-99 \%$ at $\mathrm{DQ} 324076$; north side has several patches $P$. cactus with $1 \mathrm{~m}$ framework; deep water corals (Paracyathus) collected off Ra's Shaykh.

Threats: anchor block, ropes, gill nets, fish trap, litter in coral; much dead coral.

\section{Khawr Ghubb Ali (DQ324075)}

Coral Community Type: A, B (site number 013)

Description: shore fringed by reef fused Porites, framework $1.5 \mathrm{~m}$, cover $70-99 \%$, bordered by rock with variety corals, predominantly Platygyra in shallows, bush and tabular Acropora deeper, or patches $P$. damicornis; all leafy, branching coral here and next site have extended branches.

Threats: anchor block, ropes, fish trap, litter in coral, much dead coral.

Khawr Ghubb Ali (DQ368060)

Coral Community Type: A, B (site number 014)

Description: shore fringed by Platygyra daedalea in shallows leading onto bushy Acropora, with well-developed patch $P$. cactus reef, $1 \mathrm{~m}$ framework.

\section{Khawr Bustan (DQ360105)}

Coral Community Type: B

Description: bordered by patches $P$. damicornis, talus with bushy and tabular Acropora. 
Jazirat Umm al Ghanam (DQ344126)

Coral Community Type: B (site number 015)

Description: gradual slope of tumbled rock to sand at $12 \mathrm{~m}$; between $2-10 \mathrm{~m}$ rock $60-85 \%$ covered by coral, decreasing rapidly in extent to few corals scattered over sand at $12 \mathrm{~m}$; coral predominantly flattened Porites; Stylophora also common in flattened form.

Jazirat Umm al Ghanam (DQ356167)

Coral Community Type: A, B (site number 016)

Description: bay fringed north and south by Porites reef extending from flat-topped, fused framework (1.5-2m thick) in shallows to border $2-4 \mathrm{~m}$ diameter Porites boulders; extends out of bay as scattered coral on rock.

Threats: ropes in coral, litter, sack in coral.

Jazirat Umm al Ghanam (Ra's Salib) (DQ363170-366167)

Coral Community Type: A, B (site number 017)

Description: patches fused Porites - P damicornis, scattered large Porites 2-3m diameter, Montipora, tabular Acropora; west side leading off shore dissected flat-topped fused Porites $1.5-2 \mathrm{~m}$ framework.

Threats: ropes, fish trap, gill net in coral.

Jazirat Umm al Ghanam (DQ361163-360152)

Coral Community Type: A, B (site number 018)

Description: shore fringed by continuous reef of fused Porites, $<2 \mathrm{~m}$ framework, or patches $P$. damicornis, whorled or branched Montipora, tiers tabular and bushy Acropora; scenic mix corals; cover $90-99 \%$ (live $80-95 \%$ ).

Threats: ropes, gill net, litter (cans, bottles, plywood, sheets aluminium, pipes, angle iron), raft in coral.

Jazirat Umm al Ghanam (DQ358132-352127)

Coral Community Type: A, B (site number 019)

Description: scattered large Porites (maximum $5 \mathrm{~m}$ diameter) fused in patches with extensive banks $P$. damicornis and patches large tabular Acropora, very scenic in places, cover $80-95 \%$, framework $<1.5 \mathrm{~m}$; southeast headland bare current-scoured rock; east shore rock with dense coral 60-90\% cover Porites, bushy and tabular Acropora, patches $P$. damicornis; narrow patches fused Porites in shallows, framework $1-1.5 \mathrm{~m}$; very large Tubastrea in $<1 \mathrm{~m}$ depth.

Threats: ropes, anchor, litter (cans, drums, cardboard, fabric) in coral.

Khawr al Quway' (DQ374169)

Coral Community Type: A, B (site number 020)

Description: beach fringed by solid framework fused flat-topped Porites ca. $25 \mathrm{~cm}$ thick in shallows, extends offshore reaching $1 \mathrm{~m}$ thick and becoming dissected. At ca. $1.3 \mathrm{~m}$ thickness framework breaks up into individual large Porites heads (1-2m diameter, $2.5 \mathrm{~m}$ maximum) with spaces filled by tabular Acropora and $P$. damicornis; corals scattered down gentle slope to sand at $6 \mathrm{~m}$. Same pattern followed along entire east shore of strait, 
i.e., intermittent small areas of Porites patch reef, patches of $P$. damicornis, scattered corals over rock-sand slope.

Threats: ropes, gill net, anchors in coral; tarballs.

\section{Ra's Shuraytah - Al Makhbuq (DQ380190)}

Coral Community Type: B

Description: rock boulders with scattered corals, strong currents.

Threats: $1 \mathrm{~A}$. planci (westernmost sighting) seen feeding on tabular Acropora.

\section{Khawr Fordha (DQ380170)}

Coral Community Type: A, B

Description: shallow bay bordered by coral patches extending as banks offshore in ca. $8 \mathrm{~m}$; slope gradual, alternating talus with sandy patches, $P$. damicornis patches, and $80 \%$ cover bushy and tabular Acropora to sand 7-8m; coral best developed DQ386174 Ra's Rarak. Threats: much litter, including ballast bags of Iranian small boat traders, much dead coral; many $A$. planci scars; $33 \mathrm{~A}$. planci seen in group.

\section{Ra's Rarak (DQ386174)}

Coral Community Type: A, B (site number 021)

Description: fused flat-topped Porites reef off beach, $0.5-1 \mathrm{~m}$ thick framework; north side massive fused castellated Porites to $3 \mathrm{~m}$ diameter, $2 \mathrm{~m}$ thick framework, some hollowed out; Porites $<4 \mathrm{~m}$ diameter maximum; very scenic.

Threats: fishing lines, ropes in coral, litter, tarballs ashore, many A. planci feeding scars; 1 large $A$. planci seen from surface, others tucked under coral.

\section{Khawr Khayran (DQ390160)}

Coral Community Type: A, B (site number 022)

Description: east side coral covered slope; predominantly bushy and tabular Acropora, mostly dead; shallows talus with small bushy Acropora. west side DQ389162-390166 slope covered $<95 \%$ by bushy and tabular Acropora - talus - $P$. damicornis to $8-10 \mathrm{~m}$ depth; also large patches whorled Montipora with framework 1-1.5m thick.

Threats: coral hauled up in nets, much litter (cans, bottles, linoleum, boxes, plastic bags); much dead coral; many $\mathrm{A}$. planci feeding scars east and west; $33 \mathrm{~A}$. planci seen from surface east side, 2 west, others tucked under coral.

\section{Khawr Ran (DQ410164-414169)}

Coral Community Type: B (site number 023)

Description: east shore fringed by coral; no framework development, but cover reaches ca. $70 \%$ predominantly tabular Acropora in upper 2-3m; below this coral assemblage variable and generally mixed, but poorly developed ending at $5-7 \mathrm{~m}$ on sandy slope; toward south more dead, broken, tumbled coral; $P$. damicornis predominates in patches, and is an unusual field of $1-1.5 \mathrm{~m}$ diameter "mushrooms" of isolated Astreopora colonies below Acropora zone at $3-6 \mathrm{~m}$. west shore bordered by similar coral with occasional large Porites. Threats: much litter (cans, bags, bottles, cardboard, linoleum, Iranian trader's ballast sacks); considerable devastation by $A$. planci. $26 \mathrm{seen}$ in concentrated patch (size $<55 \mathrm{~cm}$ ). 
Jazirat Abu Sir (DQ420182)

Coral Community Type: A, B (site number 024)

Description: northeast point narrow rock ledge to wall; ledge widens west with 30-40\% cover scattered mixed corals; most cove has coral-covered slope, $80-99 \%$ cover, well-mixed patches $P$. damicornis, tabular Acropora, whorled Montipora, fused castellated Porites with $1 \mathrm{~m}$ thick framework generally, but $<3 \mathrm{~m}$ one site, very scenic; south of cove (DQ420175) tumbled rock slope with patches scattered coral or 99\% cover Porites.

Threats: ropes, litter in coral; many $A$. planci feeding scars, much dead coral, $60 \mathrm{~A}$. planci seen from surface.

Jazirat Abu Sir (DQ418174)

Coral Community Type: B (site number 025)

Description: coral-covered slope, $80-99 \%$ cover Porites - tabular Acropora - P. damicornis, ends sand $12 \mathrm{~m}$.

Threats: few A. planci feeding scars, 0 seen from surface.

Khawr Kumzar (DQ420150)

Coral Community Type: A, B

Description: east side coral sparse, intermittent patches $P$. damicornis and 1 patch whorled Montipora off gravel beach DQ430146; west side coral better developed in 2 large coves DQ412145, 412155. See specific site descriptions for further details.

Threats: gill net, fish trap, lines, linoleum in coral; coral dragged up by beach seine; $A$. planci.

Khawr Kumzar (DQ417156-416148)

Coral Community Type: A, B (site number 026)

Description: southeast shore $4-5 \mathrm{~m}$ deep shelf with $60 \%$ coral cover, predominantly tabular Acropora, 50:50 live:dead; further in bay slope covered <95\% large patches whorled Montipora or $P$. damicornis separated by talus - tabular Acropora patches; inner bay north shore patches fused castellated Porites - exceptionally well-developed north: flat-topped in shallows with $1-1.5 \mathrm{~m}$ thick framework, reaching $2-3 \mathrm{~m}$.

Threats: gill net, fish trap, ropes, linoleum, freezer, much litter in coral; much dead coral; some A. planci feeding scars, 2 seen from surface, others tucked under coral.

Khawr Kumzar (DQ412145)

Coral Community Type: B

Description: coral along south shore down gradual slope to $7 \mathrm{~m}$, predominantly tabular Acropora (only 1-5\% alive, rest broken dead in situ) with patches $P$. damicornis ca. $70 \%$ dead; further north more live coral, especially tabular Acropora along edge coral band; north side similar, but narrower band coral.

Threats: very little; much dead Acropora; numerous A. planci feeding scars, 22 seen from surface in 1 group. 
"Salla" (DQ432147-440146)

Coral Community Type: A, B (site number 027)

Description: east side outer bay rock with sandy shelves, scattered coral, $10-25 \%$ cover, west of gridline DQ440 slope gradual with coral patches $<99 \%$ cover, much dead Acropora (A. planci predation), P. damicornis, bushy Acropora, castellated Porites patches ( $2 \mathrm{~m}$ diameter heads, fused forming porous framework), much Astreopora $7 \mathrm{~m}$ deep; $P$. damicornis patches inner bay; west side large patches fused castellated Porites $2.5-3 \mathrm{~m}$ diameter, flat-topped in shallows forming micro-atolls ( $<5 \mathrm{~m}$ diameter), framework $<2 \mathrm{~m}$ thick; large patch P. damicornis, whorled Montipora, abundant Astreopora.

Threats: gill net, fish trap, ropes damaging coral; much litter (cans, bottles, fabric, drum, ballast bag) killing coral; much dead coral; many $A$. planci feeding scars, 34 seen from surface east side (28 in group), 21 west side.

Khawr Ma'ili (DQ450130)

Coral Community Type: A, B

Description: shores fringed by scattered coral growth; rock walls have encrusting species and less variety; tumbled rock boulders support denser and more varied coral assemblages, or patches of $P$. damicornis. See individual accounts for details.

Threats: severe: tarballs, flotsam, litter; main base for Iranian small boat traders who anchor on corals, litter beaches, seabed.

Khawr Ma'ili (DQ442146-440132)

Coral Community Type: A, B

Description: coral covered rock slope; $20-60 \%$ cover live coral to $90 \%$ in $P$. damicornis patches; percent cover increases south of point; patch Montipora with framework ca. $1 \mathrm{~m}$ thick, cover $80-90 \%$; also patch $100 \%$ Goniopora.

Threats: extensive smashed overturned coral, talus patches; vast areas seen eaten by $A$. planci, feeding scars numerous, 34 seen from surface.

Khawr Ma'ili (DQ440131-439127)

Coral Community Type: B (site number 028)

Description: west side cove rock shelf with $<99 \%$ coral cover in healthy mixed assemblage in 1 patch bordered by talus slopes with predominantly P. damicornis; east side scattered coral heads and talus inner reaches with patches large Astreopora heads; here $30-70 \%$ cover and less consolidated than west side; south cove less coral, much rubble, $P$. damicornis over talus.

Threats: litter, sheets linoleum on coral; much dead coral; $A$. planci feeding scars, 4 seen from surface.

\section{Jazirat al Khayl (DQ455165)}

Coral Community Type: A, B

Description: north coast has rock walls and shelves with scattered corals, patches of $P$. damicornis; northwest cove shallow sandy shelf with Porites heads, talus, patches $P$. damicornis; southeast DQ464163 6m shelf with scattered Porites reaching 30-40\% cover maximum, and rock outcrops with scattered coral; 1 Porites head 5-6m diameter; south 
coast has reef framework - see next record for details.

Threats: ropes in coral; much litter underwater, base for Iranian small boat traders causing severe litter problem; numerous $A$. planci scars, 30 large $A$. planci seen from surface in small area ( $<5$ minute count).

Jazirat al Khayl South (DQ449165-462160)

Coral Community Type: A, B (site number 029)

Description: south coast generally fringed by well developed reef of Porites with extensive patches $P$. damicornis (especially in shallows, but occasionally down slope); Porites heads $(<3-4 \mathrm{~m}$ diameter) form scenic formations ending on silty rock slope at ca. $9 \mathrm{~m}$; cover $<99 \%$.

Threats: ropes in coral; much litter, tar ashore and underwater; Iranian small boat traders cause severe litter problem; many $A$. planci feeding scars, 7 large $A$. planci seen on top of coral.

Ghubbat al Khouse (DQ480130)

Coral Community Type: A, B (site number 030)

Description: areas of good coral with true framework development and cover <99\%; deeper in bay coral growth thins out, typically sparse veneer ranging from $0-30 \%$ cover; talus banks common with localized outbreaks A. planci e.g. at DQ475118.

Threats: gill nets in coral; Iranian small boat traders cause severe littering of few small beaches and adjacent seabed, e.g. at DQ460115 very heavily littered with tins, bottles, other rubbish in sea, ashore, killing coral; large areas damaged coral; A. planci.

Ghubbat al Khouse (DQ462126-460115)

Coral Community Type: A, B (site number 031)

Description: slope coral covered; varies from mixed assemblage to patches covered by whorled Montipora, Goniopora, P. damicornis, P. cactus; corals sparser deeper in bay existing only as thin veneer, amount of broken, upturned corals, talus banks, litter increases further into bay; corals sparse in head of bay.

Threats: damage to corals possibly from anchors; Iranian small boat traders causing severe litter problem underwater, ashore.

Ghubbat al Khouse (DQ500118)

Coral Community Type: B (site number 032)

Description: typical inner bay environment: primarily bare rock with small scattered corals in shallows; shore large blocks rock undercut forming deep caves - oysters all dead inner parts, alive outer rocks.

Threats: litter on seabed.

Ghubbat al Khouse (islet) (DQ490137)

Coral Community Type: B (site number 033)

Description: mainland slopes gradually from shore to sand at $5-6 \mathrm{~m}$ with occasional sheer drops, stepped ledges to $8-10 \mathrm{~m}$ east of islet; slope covered by varied corals, including patches Acropora, P. damicornis, Goniopora, Turbinaria. 
Threats: gill nets, fish trap, lines entangled and damaging coral; bags, bottles in coral.

Ghubbat al Khouse (DQ478145)

Coral Community Type: A, B (site number 034)

Description: rock slopes from base of cliff to sand at 3-5m; slope covered $<99 \%$ alternatively by tabular Acropora or $P$. damicornis with occasional large Porites heads.

Threats: numerous $A$. planci feeding scars; 14 large $A$. planci (diameter $42 \mathrm{~cm}$ ) seen from surface.

Jazirat Bu Rashid (Tawakkul) (DQ495204)

Coral Community Type: B (site number 035)

Description: series of stepped ledges and walls to $>40 \mathrm{~m}$; ledges covered by Sinularia and Sarcophyton; walls blanketed with small blue ?Xenia-type alcyonarian, scattered hard corals, purple gorgonian, yellow antipatharian, bright yellow-orange sponges, red colonial tunicates; many fishes.

Northwest Point Musandam Peninsula (DQ500154)

Coral Community Type: A, B

Description: narrow coral reef $<90 \%$ cover primarily Porites with tabular and bushy Acropora; rest of shore of this bight is rock with scattered coral and talus patches.

Threats: ca. 120 pairs trousers (Iranian trader's consignment) covering, killing coral; $A$. planci feeding scars.

Ra's al Bab (DQ503152)

Coral Community Type: $B$

Description: bay fringed by generally bare scoured rock with scattered flattened coral colonies; head of bay has large Porites and Platygyra with variety of other small corals scattered on large rock boulders on sand in ca. $5-6 \mathrm{~m}$.

Threats: current eddies concentrate tarballs and other flotsam.

Jazirat Musandam (DQ525176)

Coral Community Type: A, B (site number 036)

Description: scenic, well-developed reef of flat-topped fused and castellated Porites in north cove that runs south intermittently along entire west bight; Porites overlaid by patches $P$. damicornis, Montipora (with vertical branches in shallows, whorled down slope); zone of large Porites generally borders coral growth at $8-9 \mathrm{~m}$; reef has scenic mix corals with imposing castellated Porites separated by patches other corals.

Threats: litter in coral; abundant cans, bottles, fabric on seabed and in coral; Iranian small boat traders cause pollution problem; numerous $A$. planci feeding scars, $41 \mathrm{~A}$. planci seen from surface.

Jazirat Musandam (DQ526184)

Coral Community Type: B (site number 037)

Description: area of strong currents with scattered coral on rock; large tabular Acropora, patches 99\% Montipora (branched) in shallows. 
Threats: A. planci feeding scars, 2 seen from surface.

Jazirat Musandam (DQ535165)

Coral Community Type: A, B (site number 038)

Description: bay fringed by well-developed reef of fused Porites (flat-topped in shallows, $<4 \mathrm{~m}$ diameter), and banks talus covered by $P$. damicornis or Goniopora, large patch Echinopora; west side especially well-developed with reef-flat of compacted talus; Acropora very rare, small colonies, eaten by A. planci; reef slopes to base generally of large Porites at ca. $10-12 \mathrm{~m}$ on sand.

Threats: gill net, ropes in coral; tarballs; sand filled ballast bags, litter (plastic bags, cans, fabric) dumped by Iranian small boat traders smothering, killing, breaking coral; A. planci feeding scars.

Jazirat Musandam (DQ540162-530160)

Coral Community Type: B

Description: rock boulders with generally few small scattered corals; substrate covered by coarse gravelly sand-shell fragments with fine cover alga; water turbid eddying.

Threats: 4 gill nets entangled in coral; numerous $A$. planci feeding scars, 34 seen from surface.

Jazirat Musandam (DQ517156)

Coral Community Type: B (site number 039)

Description: large rock boulders covered by brown zoanthids, Sinularia, coral (20-50\% cover among boulders, $<80 \%$ on boulders).

Ra's Qabr al Hindi (DQ509154-516114)

Coral Community Type: A, B

Description: from Ra's al Bab south the shore has steep-stepped ledges of largely barren rock with strong current scour; the cove DQ514113 has quieter shallower water with talus banks and Porites reefs covered by Diadema, $P$. damicornis, Montipora.

Threats: current eddies concentrate tarballs, flotsam; beaches heavily polluted by oil, litter; small beaches used by Iranian small boat traders causing heavy pollution; numerous $A$. planci scars.

Ra's Qabr al Hindi (DQ518116-523110)

Coral Community Type: B

Description: tumbled rock with few scattered corals.

Threats: many A. planci feeding scars, $20 \mathrm{~A}$. planci seen from surface.

\section{Dawhat ash Shisah (DQ440060)}

Coral Community Type: A, B (site number 040)

Description: stony tidal flats with Siderastrea, Acropora, Porites, Leptastrea, Goniopora, Favites exposed low water springs; see individual site descriptions.

Threats: gill nets, lines, ropes in and dragged over coral; tarballs, flotsam, litter; A. planci. 
Dawhat ash Shisah (Daw Sunni) (DQ499105)

Coral Community Type: B

Description: coral covered slope, $80-99 \%$ cover, mixed assemblage; breaks up south toward headland and north toward beach; corals generally flattened colonies Platygyra, Echinopora, Echinophyllia typical of sheer or deeply shaded rock faces, but unusual abundance of large Astreopora colonies; patches P. damicornis.

Threats: some A. planci feeding scars.

Dawhat ash Shisah (Sharyah North) (DQ404080)

Coral Community Type: B (site number 041)

Description: patchy coral on base of dead Acropora and numerous small massive coral species; cover $30-50 \%$ live and dead coral on sand ending at $5-6 \mathrm{~m}$ in shallow sandy bay; shallow slope means coral extends further offshore than usual as a patchy thin veneer.

Threats: lines in coral; few A. planci scars in bay increasing to severe damage off north headland (90-95\% Acropora eaten by $A$.planci), 15 large $A$. planci seen from surface with many more under coral.

Dawhat ash Shisah (Jazirat Sawda) (DQ453082)

Coral Community Type: B

Description: west side slope covered $>90 \%$ P. damicornis in large patch, few Porites, bushy Acropora; cliffs toward south end and centre beside cobble beach mixed assemblage Porites and sheets Echinopora, Echinophyllia. Not studied east side.

Threats: litter underwater, $A$. planci.

Dawhat ash Shisah (DQ410055)

Coral Community Type: B (site number 042)

Description: sparse corals on rock increasing to Porites dominated assemblage towards north headland; 50-75\% cover, Porites dominated deeper with Platygyra shallower and large patches Echinopora.

Threats: ropes, anchors in coral; few $A$. planci feeding scars.

Dawhat ash Shisah (DQ402050)

Coral Community Type: B

Description: banks talus with small Acropora, $P$. damicornis; dead coral, talus abundant in shallows, more living coral scattered deeper; living coral cover increases toward headland from $25 \%$ maximum to $50 \%$ at point (of which $75 \%$ Platygyra). This pattern typical of shallow coves that characterise the west side of the bay, with few notable exceptions (e.g., Porites reef at DQ400066, coral assemblage at DQ404080 that described separately).

NOTE: visibility decreasing daily as plankton growth, bioluminescence, red tides increasing turning water khaki - aswad to locals who claim water in summer clear/abyadh; filamentous algal growth on rock proliferating.

Threats: A. planci feeding scars. 
Dawhat ash Shisah (DQ411028)

Coral Community Type: A, B (site number 043)

Description: entire west shore fringed by well-developed reef of fused flat-topped Porites with solid $1-2+\mathrm{m}$ framework.

Threats: ropes in coral; A. planci feeding scars, several seen under coral.

Dawhat ash Shisah (Marboob) (DQ425016)

Coral Community Type: B (site number 044)

Description: west side dense patches $P$. cactus, large tabular Acropora giving way to coral encrusted rock ( $<80 \%$ cover) up to and around west headland.

Threats: ropes entangled and dragged across coral; beach seine snagged some live coral; tarballs, flotsam ashore; much litter underwater; several $\mathrm{A}$. planci scars, $3 \mathrm{~A}$. planci seen from surface, others tucked under coral; beach used to careen boats, increasing litter pollution underwater, especially linoleum sheets.

\section{Dawhat ash Shisah (DQ462018)}

Coral Community Type: B (site number 045 )

Description: rocks encrusted by corals; much dead Acropora; good nursery area for variety of fishes.

Threats: flotsam, tarballs; numerous $A$. planci feeding scars clearly linked to dead Acropora; $3 \mathrm{~A}$. planci seen exposed, many others tucked under coral.

Ra's Khaysah - Ra's Bashin (DQ494017-487968)

Coral Community Type: A, B (site number 046)

Description: this stretch coast fringed intermittently by coral assemblages of different kinds; DP486982-481986 has banks talus $>1 \mathrm{~m}$ thick covered $99 \%$ by tightly packed $P$. damicornis; next coves north have narrow fringe predominantly tabular Acropora on rock with many dead patches; see specific site descriptions.

Threats: tarballs, flotsam on beaches; numerous $A$. planci feeding scars; $A$. planci cause of much dead Acropora.

\section{Ra's Khaysah South (DQ476004)}

Coral Community Type: A, B (site number 047)

Description: southeast point: slope of boulders with scattered corals; low cliffed headland between south cobble beach and north sandy beach has well developed Porites reef formed of $<3-4 \mathrm{~m}$ diameter fused Porites with $>2 \mathrm{~m}$ framework, compact, scenic castellated colony formations; few species this area, greater variety east over tumbled rock boulders.

Threats: several $A$. planci feeding scars.

\section{Ra's Khaysah South 1 (DQ481986-486981)}

\section{Coral Community Type: B}

Description: rock boulders with $75 \%$ cover coral (of which ca. $80 \%$ tabular Acropora); much coral dead.

Threats: numerous $A$. planci feeding scars, 2 seen from surface, others tucked under coral. 
Ra's Bashin South (DQ485968)

Coral Community Type: B (site number 048)

Description: rock slope covered $80-90 \%$ by coral; tabular Acropora predominant to $3 \mathrm{~m}$; mixed assemblage below with predominance of massive corals. (Note: north side Ra's DP488968 patch of coral covered slope, $<80 \%$ cover, mixed predominantly massive corals).

Threats: ropes in coral; large devastated area coral, much dead Acropora (possibly $A$. planci related), numerous $A$. planci scars, many $A$. planci seen tucked under coral, 0 seen from surface.

Jazirat Umm al Fayyarin (DQ548956)

Coral Community Type: B (site number 049)

Description: island drops rapidly to great depth, but there are few narrow shelves where colonies Porites, Platygyra, Symphyllia established; elsewhere rock walls and steep slopes have scattered corals ( $<40 \%$ cover maximum); much yellow antipatharian and purple gorgonian; Dendronephthya; much Tubastrea, Dendrophyllia common.

Threats: ropes and large gill net spread over reef; flotsam, grease in eddies around island; abundant $A$. planci, 63 seen from surface in $50 \mathrm{~m}$ stretch east coast including 5 on 1 Platygyra colony, many others tucked under coral, some large $A$. planci, most small $18-24 \mathrm{~cm}$ diameter.

\section{Ghubbat Shabus (DQ460960)}

Coral Community Type: A, B (site number 050)

Description: some well-developed coral communities in bay, generally in areas open to north or east; elsewhere corals occur as scattered colonies on rocky slopes (seldom $>30 \%$ cover); north part of bay has very little coral; Porites clearly dominates as primary structural component where there is framework development; the double bays from DP437958-436951 are fringed by flat-topped Porites fused to form 1-1.5m thick solid framework, with few other corals; see specific site descriptions for details.

Threats: gill nets, ropes in coral; litter underwater; $A$. planci.

Ghubbat Shabus (DP440957)

Coral Community Type: B (site number 051)

Description: rock slope with ledges covered $50-65 \%$ coral, $80 \%$ maximum; predominantly Acropora on flat shelf with walls covered by sheets different corals; well mixed diverse assemblage; no sign $A$. planci.

Ghubbat Shabus (DP455940)

Coral Community Type: B

Description: rock slope with sand and scattered corals, cover seldom $>30 \%$.

Threats: trash on corals, including drum; $A$. planci feeding scars.

Ghubbat Shabus (DP460940-466938)

Coral Community Type: A, B

Description: east bay: coral better developed west side; Porites dominated but patchy; east 
side steeper/sheer with scattered coral on rock; west bay: sheer east side $25-50 \%$ cover coral on rock; head bay shallow below stony tidal flat; outer west side patches reef development dominated by Porites.

Threats: gill net in coral.

Ghubbat Shabus (DP475925-480916)

Coral Community Type: A, B (site number 052)

Description: this double bay fringed by dense well-developed coral community; Porites dominant as scenic, castellated, multicoloured colonies; patches covered by Goniopora (entire slope), $P$. damicornis, Acropora, large sheets Echinopora; reef framework reaches $1.5 \mathrm{~m}$ thick in places, best developed east bay and east side west bay; west side west bay coral continues as thin veneer over rock, breaking up towards headland into rock with patchy coral.

Threats: ropes, gill net, anchors in coral; anchor damage inner bay; litter in coral; several $A$. planci feeding scars, $1 \mathrm{~A}$. planci seen from surface, many others tucked under coral.

Ra's Dillah North (DP486909)

Coral Community Type: B (site number 053)

Description: much sheer primarily bare rock around Ra's with scattered sheets of coral; small coves have shelves with some coral; 1 site snorkelled: 10-70\% cover Sinularia, less cover live corals; highest cover over large rock blocks; scattered Porites, Platygyra over rock-sand shelf.

Threats: gill net in coral; wastage fishes (discarded dead fishes include sohal, angelfish, garfish); few A. planci feeding scars, 1 A. planci seen from surface.

\section{Ra's Dillah South (DP488904)}

Coral Community Type: B

Description: sheer wall off point, shelf in first small cove with huge boulders leading to slope of large tumbled boulders with caves down to $>40 \mathrm{~m}$; scattered corals shallower to scattered purple gorgonian and yellow antipatharian deeper; 1 huge black coral (bushy grey).

\section{Khawr Habalayn (DP400900)}

Coral Community Type: A, B (site number 054)

Description: outer bay has steep/sheer rock walls with sheets encrusting corals, small shelves below wadis have limited coral growth; central bay prolific coral growth, dense patches $P$. damicornis, Acropora, fused Porites reefs; inner bay dense cover bushy Acropora in places, generally scattered coral with Platygyra predominant; DP945911 P. damicornis patches; DP437910 west side luxuriant tabular Acropora, east side cliffed, sheer; bays north of point at DP411925 lined by bushy Acropora, P. damicornis patches - also patches in centre shallow bays; Ghubbat al Ghazirah bordered predominantly Platygyra with $P$. cactus, small patches fused Porites, DP362953 80-99\% cover predominantly Platygyra shallows, $P$. cactus slope and small patches bushy Acropora ( $P$. cactus not as extended leaves as Khawr ash Shamm). See specific site descriptions for details. 
Threats: gill net, fish trap, fishing lines, ropes in coral; litter ashore, underwater; much dead coral; severe coral damage from $A$. planci.

Khawr Habalayn (DP474906-473911)

Coral Community Type: A, B (site number 055)

Description: fused flat-topped Porites reef, framework 1-3m, Porites diameter 2-3m, banks $P$. damicornis, sheets Echinopora; very scenic, castellated, colourful Porites.

Threats: gill net, fishing lines, rope in coral; litter underwater; much dead Acropora; many A. planci feeding scars, 3 seen from surface, many tucked under coral.

\section{Khawr Habalayn (DP425911)}

Coral Community Type: A, B (site number 056)

Description: 10/04/86 beach fringed Acropora - P . damicornis west-centre, east Montipora with $<1 \mathrm{~m}$ thick framework; east headland tumbled rock slope with luxuriant tabular Acropora; further out cliffs extend underwater as rock walls with narrow ledges; sheer faces encrusted large sheets coral, 30cm Tubastrea. 13/01/90 Acropora areas off beach, headland wasteland of dead, broken coral, talus, some recolonisation outer bay and deeper edge; small $P$. damicornis patches off headland with $A$. planci scars; $P$. damicornis on dead Acropora off beach large dead patches (flood runoff or A. planci?).

Threats: litter underwater (cans, linoleum); A. planci predation: 10/04/86 27 seen from surface in 4,000 square metres, estimated 25\% Acropora dead; 13/01/90 total devastation Acropora, A. planci still present, 3 seen from surface, others tucked under coral, feeding on $P$. damicornis off headland, Montipora off beach.

Khawr Habalayn (DP414950)

Coral Community Type: B (site number 057)

Description: slope, rock shelves covered $40-95 \%$ coral, predominantly bushy Acropora; large coral-encrusted blocks separated by narrow, sheer gullies; deeper shelves deeply undercut.

Threats: litter underwater; much dead Acropora and A. planci feeding scars; huge $A$. planci all tucked under coral.

Khawr Habalayn (Maksar) (DP395977)

Coral Community Type: B (site number 058)

Description: 1:1 sandy slope with ca. $40 \%$ coral cover, deeply undercut rock shelves with ca. $80 \%$ cover; predominantly Platygyra (no white patch disease like across ridge in Khawr ash Shamm).

Threats: some litter.

Khawr Habalayn (Jazirat Habalayn) (DP358938)

Coral Community Type: A, B (site number 059)

Description: island surrounded by coral, particularly well developed Porites reef northwest and north sides, $2-3 \mathrm{~m}$ diameter colonies, $2-3 \mathrm{~m}$ thick framework, patchy Porites reef elsewhere, large field bushy Acropora on wide shelf to south; cover $50-90 \%$ generally, 99\% on Porites reefs and dense Acropora patches; west side assemblage 
Porites-Stylophora-Platygyra-Acropora (bushy and large tabular).

Threats: ropes in coral; many A. planci feeding scars and devastated coral, 117 seen from surface in 15 minutes south side where devastation worst, many others tucked under coral; largest A. planci $57 \mathrm{cms}$ diameter. $28 \mathrm{Apr} 90$ recount $A$. planci: shallow Acropora on south shelf nearly all dead, fringes have living coral and numerous $A$. planci scars; most $A$. planci now deep or tucked under coral - not clustered as previously; $63 \mathrm{~A}$. planci counted from surface in 25 minutes.

Khawr Habalayn (Habalayn) (DP327930)

Coral Community Type: A, B

Description: patches fused Porites off village; framework 1-1.5m thick DP330427.

Khawr Habalayn (Khawr Najd) (DP340903)

Coral Community Type: A, B (site number 060)

Description: coral reef similar appearance Khawr Shamm reefs dominated by large-blade Pavona, $>1 \mathrm{~m}$ thick framework; flat-topped, porous Platygyra in shallows.

Threats: anchor block in coral.

Khawr Habalayn (Nibah) (DP420850)

Coral Community Type: A, B (site number 061)

Description: fused flat-topped Porites reef with bushy and tabular Acropora from DP430853 intermittently into bay to islet DP415848; inner bay small patch Acropora as thin veneer DP412865; DP414866 sand-rock slope covered $80-90 \%$ by small bushy Acropora, some large dead tabular Acropora; scattered Astreopora on sand off seaward edge.

Threats: flotsam, bottles, broken glass, old tarballs ashore; 11/04/86 8 large $A$. planci seen from surface in 3000 square metres DP414866, possibly cause of dead Acropora tables, feeding scars seen from boat 13/01/90.

Khawr Habalayn (Nibah East) (DP430854)

Coral Community Type: A, B (site number 062)

Description: fused, flat-topped, porous Porites reef of $2-2.5 \mathrm{~m}$ diameter colonies, $1.5-2 \mathrm{~m}$ thick framework.

Threats: some obvious A. planci predation of bushy Acropora; no A. planci seen.

Khawr Habalayn (Yidiyah al Bahr) (DP438858-445860)

Coral Community Type: A, B

Description: flat-topped fused Porites reef ca. 300m long DP443856; patch bushy and tabular Acropora below cobble beach DP438854 with few A. planci scars; west shore Platygyra-Acropora (bushy, tabular) assemblage.

Threats: A. planci feeding scars.

Khawr Habalayn (DP455856-472855)

Coral Community Type: B

Description: steep to sheer rock walls with scattered sheets encrusting coral (majority this 
section coast); shelves, rocky slopes denser coral assemblages; Platygyra-Porites-Acropora (bushy, large tabular) assemblages DP455856, 466854 cover $80-90 \%$, and 472855 cover $65-80 \%$.

Threats: A. planci feeding scars and $2 \mathrm{~A}$. planci seen from boat each site.

Ra's Sarkan - Ra's Secun (DP477850-440808)

Coral Community Type: B

Description: small coves have shelves 3-6m deep with rock boulders, few scattered corals ( $<5 \%$ cover usual, to $20-50 \%$ maximum in small discrete patches); shelves bordered by walls ( $<1 \%$ coral cover); Dendronephthya, Tubastrea from $2 \mathrm{~m}$ down.

\section{Dawhat Qabal (DP400800)}

Coral Community Type: A, B (site number 063)

Description: relatively shallow bay with rocky shore ending on sand at $3-6 \mathrm{~m}$; north coast has few scattered corals, small isolated patches $P$. damicornis; coral growth best along south coast where Porites fuse to form shore-fast, flat-topped reef framework 1-2m thick, exposed low water springs; framework reef at DP396784 (small), DP400787 (ca. 400m long), DP407783 (ca. 250m long), DP411781 (small), DP412782 (small), DP413783 (small), DP416782; also large patches $P$. damicornis, mixed coral assemblages $(<60 \%$ cover in bay DP406785); small patch castellated Porites DP387798.

Threats: fish traps, gill nets, ropes, poles in coral.

Dawhat Qabal (DP415782)

Coral Community Type: A, B (site number 064)

Description: sheer gullied rock each side wadi fan; rock boulder slope below fan covered by coral, predominantly Porites and Platygyra forming fused, flat-topped, porous $1.5 \mathrm{~m}$ thick framework; Porites $1.5-2 \mathrm{~m}$ diameter; large patch $H$. exesa down slope.

\section{Ra's Marovi (DP430743)}

Coral Community Type: B (site number 065)

Description: an apron of sand slopes north and south from the narrow tidal bar joining the west island to the mainland; north slope covered by rock, mixed corals, Sinularia; south slope has patches $P$. damicornis bordered by walls with fringe tumbled rock, coral cover 25-75\%; north island sheer wall with large colony purple gorgonians, large Tubastrea, much Dendronephthya; Dendronephthya abundant all around island in orange, mauve colours; Sinularia also abundant and predominant on south side shelves; southeast shelf large with scenic, colourful mix corals; parts south sheer, deeply undercut walls.

Threats: gill nets, ropes in coral; corals and small lobsters in tangle net; few cans, bottles on seabed; many $A$. planci feeding scars, 1 seen exposed, others tucked under coral.

\section{Ma'awi (DP404731)}

Coral Community Type: A, B (site number 066)

Description: large patch tiered Acropora (1-1.5m thick framework, 2-3m diameter tables, $100 \%$ cover with sand patches, $95 \%$ live, extends from shore to fringe Sinularia, sand $4 \mathrm{~m}$ depth; other corals confined to edges, sandy patches. Similar smaller table Acropora patch 
DP410741.

Limah (DP425700)

Coral Community Type: B (site number 067)

Description: north end beach DP425708 small patch coral Porites $(<2.5 \mathrm{~m}$ diameter), $P$. damicornis, tabular Acropora predominant.

Threats: anchor block, lines, ropes, gill net, fabric, plastic bags, cans in coral; large patch of long-dead big tabular Acropora; some A. planci scars, 0 seen from surface - all tucked under coral.

Jazirat Limah (DP468695)

Coral Community Type: B

Description: walls to $>30 \mathrm{~m}$ cut by fissures, overhangs and caves; shelves with large rock boulders forming arches, tunnels; scattered hard corals, abundant purple gorgonians and yellow antipatharian, Sinularia and Sarcophyton; many fishes.

Threats: ropes, gill net in coral.

Ra's Limah South (DP440687)

Coral Community Type: B

Description: extensive patches $P$. damicornis fill cove, inner side large heads $P$ orites, rock with scattered corals.

Threats: gill net in coral.

Ghubbat Aqabah (DP400670)

Coral Community Type: A, B (site number 068)

Description: shallow bay with tumbled rock or low cliffed shore to sand; wherever shallow rock shelves (i.e. below wadis, in coves) may be some Porites reef development or dense cover by bushy and tabular Acropora which now dead and secondarily colonized by other corals (especially $P$. damicornis); extensive dead coral, talus banks.

Threats: severe: lines, ropes, gill nets, fish traps, cans, fabric, bottles, drums, plastic bags and sheets in coral; extensive dead coral; A. planci.

Ghubbat Aqabah (Ra's Samut) (DP437677-424680)

Coral Community Type: B (site number 069)

Description: talus banks on shelves, much dead bushy, tabular Acropora on rock and down slope to sand $4 \mathrm{~m}$; coral on rock walls and boulders below healthy (cover 3-50\%); small cove (DP434681) has slope carpeted by Goniopora (ca. 80\% cover).

Threats: severe: 4 gill nets, ropes, lines, poles entangled and damaging coral; 4 drums, cans, bottles, plastic in coral; many $A$. planci feeding scars, all $A$. planci tucked under; much dead Acropora.

Ghubbat Aqabah (Aqabat) (DP416676)

Coral Community Type: A, B

Description: small solid patch fused Porites reef leading from flat-topped reef-flat $(0.5-1 \mathrm{~m}$ thick framework) down short slope (1.5m maximum framework) to large knobbed Porites 
heads on sand in $3 \mathrm{~m}$; Porites $<2 \mathrm{~m}$ diameter hollowed in places; dense small $P$. damicornis banks seaward.

Threats: gill net, ropes in coral; much dead bushy and tabular Acropora.

Ghubbat Aqabah (Al Haqil) (DP394686)

Coral Community Type: B

Description: shallow sand with rocky patches, extensive area of diffuse large tabular Acropora and Porites colonies, no cohesive reef structure; ca. 50\% cover.

Ghubbat Aqabah (DP395649)

Coral Community Type: A, B (site number 070)

Description: Porites fused to form flat-topped tidal platform, ends sand $1-1.5 \mathrm{~m}$, highly porous hollowed out and solid framework.

Threats: lines, fish trap in coral.

Sidwen (DP400628)

Coral Community Type: B

Description: south shore fringed by band Porites, $P$. damicornis patches, tabular and bushy Acropora; 30-75\% cover.

Massar (DP375604)

Coral Community Type: B (site number 071)

Description: tiers of tabular Acropora on rock, $2-2.5 \mathrm{~m}$ diameter $>80 \%$ cover.

Threats: rope in coral; many $A$. planci feeding scars; $1 \mathrm{~A}$. planci seen from surface, others tucked under coral; patches broken tabular Acropora.

East Coast Musandam (DP382589)

Coral Community Type: A, B (site number 072)

Description: fused, flat-topped Porites ( $<3 \mathrm{~m}$ diameter) reef, $1-1.5 \mathrm{~m}$ thick framework; outer bay scattered Porites and $P$. damicornis patches; dense banks $P$. damicornis north side; beautiful coral garden south side.

Threats: ropes, gill net, fish trap, anchor in coral.

Dawhat Sharyah (DP370575)

Coral Community Type: A, B

Description: DP370578 shallow shelf with large patch bushy and tabular Acropora; DP365573 shallow fused, flat-topped Porites reef, 1-1.5m thick framework.

East Coast Musandam (DP369563)

Coral Community Type: A, B (site number 073)

Description: fused, flat-topped Porites (3-3.5m diameter) reef, 1-1.5m thick framework; continues offshore as tiers tabular Acropora ( $2 \mathrm{~m}$ diameter) and rock covered $80 \%$ by corals.

Threats: gill net in coral; numerous $A$. planci feeding scars and dead tabular Acropora southeast side cove; $1 \mathrm{~A}$. planci seen from surface, others tucked under coral. Porites in 
shallows pitted by Echinometra.

Sanat (DP357543)

Coral Community Type: A, B (site number 074)

Description: east shore has banks of $P$. damicornis, and bushy and tabular Acropora; west shore has extensive fused Porites reef fringing almost entire length with bushy and tabular Acropora, P. damicornis along reef edge; $1-3 \mathrm{~m}$ thick framework, $2-4 \mathrm{~m}$ diameter Porites. Threats: gill net, fish trap, ropes in coral; litter underwater, tarballs; much dead tabular Acropora; shallow Porites pitted by Echinometra.

Khawr Mala (DP345530)

Coral Community Type: B

Description: shore fringed by bush and tabular Acropora, especially outer half east shore.

\section{Dawhat Haffah (DP310480)}

Coral Community Type: A, B (site number 075)

Description: DP298474 very solid narrow finger Porites reef, $0.5-1.5 \mathrm{~m}$ thick framework; much sponge; DP298474 and 311475 narrow Porites reefs.

Threats: gill net, fish trap, ropes, anchor blocks, fabric, plastic bags, cans in coral.

\section{Zaghi (DP272435)}

Coral Community Type: B (site number 076)

Description: south headland has rocky shore with rock slope and large rock blocks underwater; parts covered by dense coral, predominantly tabular Acropora, Porites.

Threats: severe damage by gill nets, ropes; litter/flotsam on beach and underwater, many tarballs.

\section{Zaghi South (DP276416)}

Coral Community Type: B (site number 077)

Description: south side beautiful coral garden; tiers tabular Acropora $(<2.5 \mathrm{~m}$ diameter), 95\% cover.

Threats: gill net, ropes in coral.

Ra's Sawt North (DP279410)

Coral Community Type: B (site number 078)

Description: north facing shore from cobble beach to point is fringed by tiers of tabular Acropora, densest below beach, sparser toward point.

Threats: 2 large gill nets flat over coral (now dead/damaged); many ropes in coral.

Daba North (DP272400)

Coral Community Type: B (site number 079)

Description: north end beach bordered rock shore with scattered corals on rock boulders in shallows; further offshore and around headland 50-65\% cover coral, predominantly tabular Acropora (1.5-2m diam) mixed with P. daedalea and Porites.

Threats: numerous gill nets, ropes and fishing lines, fish traps in coral. 
Ra's Sawadi (EM790295-814280)

Coral Community Type: A, B (site number 080)

Description: assemblages of coral colonies on rock occur around the smaller islands, off the north and west sides of Maqbarah, and along the north coast of Jabal 'Add, forming a few small patches of coral framework $<1 \mathrm{~m}$ thick; finest coral (highest percentage living cover, least damage, most extensive area), Stylophora-P. damicornis-Acropora (tabular and bushy) assemblage off western island, but heavily infested with $A$. planci (13 counted from surface in 2 minutes).

Threats: considerable coral breakage in shallows around central islands caused by recreational boaters.

\section{Daymaniyat Islands (General) (EM967380-FM200370)}

Coral Community Type: A, B (site number 081)

Description: many well-developed framework reefs (generally $1-3 \mathrm{~m}$ thick, but reaching $4 \mathrm{~m}$ ) formed of Porites and $P$. damicornis; typically north shores have cliffs falling to tumbled rocky slopes between 6 and $25 \mathrm{~m}$ deep with abundant coral growth; southern shores shallow, often with banks of $P$. damicornis forming ribbons of reef parallel to shore at depth of 6-8m; clearly area of former luxuriant coral growth, but many large Acropora, Porites, Symphyllia colonies now dead; amount of dead coral (cover up to 99\%) is a remarkable feature of many of the reefs; one patch Galaxea south of Jun forms framework reef $40 \times 10 \mathrm{~m}$ and $1 \mathrm{~m}$ thick - unique in Oman.

Threats: ropes, fishing lines, gill nets in corals causing considerable damage; collection Lambis truncata and Pinctada by fishermen for sale to people on mainland; A. planci; collection shells and lobsters and spearfishing by expatriates.

Daymaniyat Islands (Clive Rock) (EM967380)

Coral Community Type: A, B (site number 082)

Description: large, predominantly sand-covered shoal with largely dead vestiges of former framework reefs scattered over it and around the rim; shoal slopes from ca. $8 \mathrm{~m}$ down talus-strewn slopes except in northeast where rock rim drops sheer to sand at $20 \mathrm{~m}$; rim of north slope has largely dead and dissolving framework reef with numerous small heads of Porites (not clear whether these are vestiges of former colonies or new growth).

Threats: gill net in corals.

Daymaniyat Islands (West Jun) (EM987366)

Coral Community Type: A, B (site number 083)

Description: large rock outcrop with slopes to $15-20 \mathrm{~m}$ and sheer drop to $23 \mathrm{~m}$; few scattered corals; southwest slope rock-sand 60\% coral 40\%, much dead tabular Acropora (formerly huge colonies $>3 \mathrm{~m}$ diameter), Porites framework reef $75 \%$ dead.

Daymaniyat Islands (East Jun) (FM000364)

Coral Community Type: A, B (site number 084)

Description: Well-developed reef of large fused Porites with 3-4m thick framework fringes southeast shore; several large banks $99 \%$ live $P$. damicornis $2-3 \mathrm{~m}$ thick south of cut between this and next island east. 
Threats: gill nets, ropes in coral, one net laying flat over vast area, another rolled up with coral bouncing over reef causing extensive damage; spearfishing, lobster catching by Naval personnel.

\section{Daymaniyat Islands (Island east of Jun) (FM001364)}

Coral Community Type: A, B (site number 085)

Description: Porites framework reef ( $2 \mathrm{~m}$ thick) southwest side island, narrows east, breaks up onto talus slope with large dead tabular Acropora; remarkably few other corals on reef; low banks $P$. damicornis offshore.

Threats: A. planci, 3 seen exposed eating Porites.

Daymaniyat Islands (western central island) (FM076384-093387)

Coral Community Type: A, B (site number 086)

Description: shallow south side with numerous patches and scattered heads of coral, some small framework reefs ( $<1 \mathrm{~m}$ thick) formed of $P$. damicornis, Acropora, Montipora, banks of $P$. damicornis further offshore; north side deeper with abundant coral along tumbled rock base of cliffs, framework reefs in east bay.

Threats: extensive damage to corals from gill nets.

Daymaniyat Islands (west, western central island) (FM076384)

Coral Community Type: B (site number 087)

Description: sheer rock drop to $20 \mathrm{~m}$ from rock ridge $(6 \mathrm{~m})$; generally small corals, numerous bases large, long-dead, tabular Acropora; vertical faces encrusted Tubastrea and variety sessile invertebrates.

Threats: gill net in coral, large gastropods entangled.

Daymaniyat Islands (northwest, western central island) (FM077384)

Coral Community Type: B (site number 088)

Description: vertical wall to tumbled rock from 6-15m; abundant corals; vertical walls encrusted Tubastrea; large patch Goniopora (99\% cover); shallower surfaces (3-8m depth) large rock blocks covered $>95 \%$ by encrusting Montipora, Porites, Platygyra, Acropora (bushy); much long-dead, tabular Acropora.

Daymaniyat Islands (east point, western central island) (FM090387)

Coral Community Type: B (site number 089)

Description: east point has rock ledge with abundant bases of large, long-dead, tabular Acropora dropping vertical to sand at $14 \mathrm{~m}$.

Threats: gill net caught in corals along wall with entangled remains of hawksbill turtle.

Daymaniyat Islands (northeast bay, western central island) (FM090385)

Coral Community Type: A, B (site number 090)

Description: northeast bay filled with flat-topped fused Porites reef (framework 1-1.5m thick), forms mosaic over sand (coral 80\%:20\% sand). 
Daymaniyat Islands (island west of main island) (FM103386-112390)

Coral Community Type: A, B (site number 091)

Description: framework reefs of $P$ orites northeast and $P$. damicornis southeast; remarkable amount of dead coral.

Threats: ropes, gill nets, fish traps entangled in and killing coral.

Daymaniyat Islands (north, island west of main island) (FM106390)

Coral Community Type: A, B (site number 092)

Description: rock with extensive dead coral, including abundant bases of long-dead, tabular Acropora, Porites, Symphyllia; east side this bay corals abundant, Porites forms reef framework $>3 \mathrm{~m}$ thick.

Threats: net in corals.

Daymaniyat Islands (south, island west of main island) (FM111386)

Coral Community Type: A, B (site number 093)

Description: series of banks parallel to shore of living $P$. damicornis with few other corals; shore-fast rock reef with scattered corals leading west to extensive area large dead tabular Acropora, Porites.

Threats: gill nets, fish traps entangled in and killing coral.

Daymaniyat Islands (main island) (FM116393-131391)

Coral Community Type: A, B (site number 094)

Description: rocky coast of island supports abundant coral growth, but much currently dead; several small framework reefs of Porites, with best example in bay southeast of guard house.

Threats: A.planci; ropes, nets in coral; collection shells (Lambis truncata and Pinctada) by fishermen for sale to people on mainland.

Daymaniyat Islands (southwest, main island) (FM118388)

Coral Community Type: A, B (site number 095)

Description: large talus patches with small scattered coral colonies, much dead coral in shallows (particularly tabular Acropora); patches and low banks $P$. damicornis at $5 \mathrm{~m}$ depth and out.

Daymaniyat Islands (northwest, main island) (FM116394)

Coral Community Type: B (site number 096)

Description: rock outcrops and ledges with coral cover varying from scattered $(<10 \%)$ to dense $(>60 \%)$ in small patches, drops sheer to sand at $15 \mathrm{~m}$.

Threats: A. planci, one seen exposed; ropes, nets in coral.

Daymaniyat Islands (rock patches south main island) (FM126387)

Coral Community Type: B (site number 097)

Description: rock outcrop (5m deep top to $10 \mathrm{~m}$ sand) festooned with long-dead bases of large, tabular Acropora, many small coral colonies; north slope carpeted by P. damicornis.

Threats: A. planci, one seen exposed 
Daymaniyat Islands (south, main island) (FM128389)

Coral Community Type: A, B

Description: large well-developed framework reef (4-5m thick) of huge Porites; rock shelf in shallows covered $>75 \%$ bushy Acropora with large dead tabular forms; banks $P$. damicornis ( $>2 \mathrm{~m}$ thick) around seaward edge.

Daymaniyat Islands (southeast, island east main island) (FM134393)

Coral Community Type: A, B

Description: shallow basin rimmed by rock, washed by strong current; scattered corals on rock with long-dead bases huge tabular Acropora, fused Porites reef (framework ca. $4 \mathrm{~m}$ thick) borders south rim, large patch Psammocora west.

Daymaniyat Islands (west, east island main group) (FM134394-143395)

Coral Community Type: A, B (site number 098)

Description: current-scoured smooth rock surface largely devoid of corals west side channel; patch Porites framework reef ( $>2 \mathrm{~m}$ thick) on west side; abundant corals along south rocky reef between surface and $15 \mathrm{~m}$.

Threats: A. planci, 1 seen exposed.

Daymaniyat Islands (east, east island main group) (FM143395)

Coral Community Type: B (site number 099)

Description: steep drop over narrow ledges to sand $22-27 \mathrm{~m}$ depth; corals more abundant south of east point than north side, but these small and relatively low cover, rock densely covered by sponges, tunicates, purple gorgonians, yellow antipatharians, Dendronephthya. Threats: A. planci, 17 seen exposed, 1 feeding on Dendronephthya; net fish trap in coral.

Daymaniyat Islands (New Reef) (FM150360)

Coral Community Type: A, B (site number 100)

Description: large rock-sand shoal with rock outcrop from 4-5m deep to $>25 \mathrm{~m}$; south and west sides sheer, north and east sandy platform with coral patches, Porites reef framework (1-1.25m thick), abundant living coral.

Threats: nets, fish trap in coral.

Daymaniyat Islands (Kharabah) (FM190367-200370)

Coral Community Type: A, B (site number 101)

Description: south: series of banks (2-4m thick) of $>85 \%$ living $P$. damicornis $200-300 \mathrm{~m}$ offshore, $8-11 \mathrm{~m}$ deep, alternating with rock covered by dead tabular Acropora, and talus slopes; east side: rock-sand shelf with scattered solitary Porites heads (1-1.5m diameter) and large patch $P$. damicornis leading onto a well-developed but small framework reef of fused Porites (ca. $2 \mathrm{~m}$ thick) along west side emergent rocks; extensive rock platform east with scattered corals (predominantly exhibiting encrusting or small robust growth forms); alcyonarians (Sarcophyton, Sinularia) carpet the rocky ridges and eastern rim of this reef which drops sheer over a number of ledges to sand at $24 \mathrm{~m}$; north side: coral best developed along the north slope of the reef (see next account); northwest and west sides: considerable dead coral here including large colonies of Symphyllia, Acropora (tables). 
Threats: A. planci, ropes, nets in coral.

Daymaniyat Islands (Kharabah) (FM194371)

Coral Community Type: A, B (site number 102)

Description: gentle slope away from island with shelf at $13 \mathrm{~m}$ depth with many scattered corals; at $8-10 \mathrm{~m}$ depth is large area Porites reef extending east-west and forming $>1 \mathrm{~m}$ thick framework; below this is a band of $>75 \%$ dead coral.

Threats: net in corals; A. planci, 6 seen exposed in short stretch.

Fahal Island (FM530195)

Coral Community Type: A, B (site number 103)

Description: island surrounded by diverse coral communities, including incipient $P$. damicornis patch reefs ( $2-3 \mathrm{~m}$ thick framework), and mixed assemblages of smaller coral colonies on rock; abundant deep water corals on rock outcrops.

Threats: gill nets, ropes, fish traps, fishing lines, litter, boat anchors in and damaging coral; coral and shell collection; heaviest diving activity in Oman; A. planci.

Fahal Island Southeast (FM530194)

Coral Community Type: A, B (site number 104)

Description: $P$. damicornis reef in 6-8m (2-3m thick framework) sloping to border Porites boulders; rock shelf at $12 \mathrm{~m}$, dead coral and patchy small colonies living corals.

Threats: boat anchors, ropes, gill nets, fish traps in coral; conspicuous A. planci feeding scars on $P$. damicornis, 3 exposed, others tucked under coral.

Fahal Island West (FM529195)

Coral Community Type: B (site number 105)

Description: rock shelf with gentle slope ( $6 \mathrm{~m}$ deep) covered by abundant small corals (flattened, encrusting); at $10 \mathrm{~m}$ drops vertically to sand at $18 \mathrm{~m}$.

Threats: anchors, ropes, nets in coral; A. planci.

Fahal Island (Shallow Reef) (FM536199)

Coral Community Type: B (site number 106)

Description: two large rock outcrops encrusted with sponges, molluscs, corals, alcyonarians.

Threats: heavily dived - located by dragging anchors; gill net draped over.

Fahal Island (Deep Reef) (FM536199)

Coral Community Type: B (site number 107)

Description: large, deep $(22-42 \mathrm{~m})$ rock outcrop covered by deep water corals and alcyonarians.

Threats: divers locate by dragging anchors.

Ra's al Hamra North (FM527162)

Coral Community Type: B (site number 108)

Description: low rocky ridges $13-15.5 \mathrm{~m}$ deep with numerous sponges, gorgonians, 
crinoids, zoanthids, alcyonarians, and corals; Siderastrea, Anomastrea large and common; sole location Acanthastrea maxima with $>8 \mathrm{~cm}$ diameter calyces.

Threats: gill nets set in vicinity, overturn corals.

Darsayt (FM580148)

Coral Community Type: A, B (site number 109)

Description: Rock outcrop with mixed corals from $3-4 \mathrm{~m}$ to sand at $14 \mathrm{~m}$, surrounded by fringe huge Turbinaria; $P$. damicornis forms banks with $1-2 \mathrm{~m}$ thick framework southeast and in bay.

Threats: gill nets; $A$. planci.

Aint East (FM588146)

Coral Community Type: B (site number 110)

Description: generally steep rock to cliff extending as tumbled rock underwater with corals reaching $85 \%$ cover in places

Threats: nets on reef; collection of corals.

Kalbuh (FM617136)

Coral Community Type: A, B (site number 111)

Description: rock with corals from shore across bank dead $P$. damicornis to border large Porites boulders $(<3-3.5 \mathrm{~m}$ diameter, some fused to form framework) alternating with mixed Porites-P. damicornis and Porites-Platygyra, drops abruptly to sand at $5 \mathrm{~m}$ with patches $P$. damicornis; extremely scenic coral garden with Acropora (bushy and tabular) predominant.

Threats: heavily used by divers, especially for training, some breakage; ropes, fishing lines, gill net, abundant litter in coral.

Muscat Island (East Coast) (FM632137-634132)

Coral Community Type: A, B (site numbers 112, 113)

Description: rocky coast with well-developed coral communities in coves northeast, southeast and along south shore; massive corals fused to form small patches framework reef; $P$. damicornis forms banks along south shore with $2-3 \mathrm{~m}$ thick framework.

Threats: ropes, gill net in corals.

Cemetery Bay (North Bay) (FM633122)

Coral Community Type: A, B (site number 114)

Description: dense coral cover (Acropora, Porites) flanks shores, better developed north shore; small bank largely dead $P$. damicornis toward mouth bay with $1-1.5 \mathrm{~m}$ thick framework.

Threats: much dead coral.

Cemetery Bay (East Cove) (FM635119)

Coral Community Type: A (site number 115)

Description: small reef formed of huge fused Porites (3-5.2m diameter) with reef-flat secondarily colonized by other corals; $3 \mathrm{~m}$ thick framework. 
Cemetery Bay (Pillar Rock) (FM637121)

Coral Community Type: B (site number 116)

Description: rock walls and boulders encrusted by sheets Leptastrea and variety small corals from surface to sand at $21 \mathrm{~m}$.

Cemetery Bay (Headland South) (FM638117)

Coral Community Type: B (site number 117)

Description: cliffs to underwater terrace dropping sheer to sand at $18-20 \mathrm{~m}$ covered by scattered coral colonies.

Cat Island (FM642092)

Coral Community Type: A, B (site number 118)

Description: islet slopes over tumbled rock with scattered corals to $13 \mathrm{~m}$ on seaward side; rock shelf with dense cover of alcyonarians and corals, fused Porites and banks $P$. damicornis border rest of islet; $P$. damicornis bank southeast forms $1-1.5 \mathrm{~m}$ thick framework and extends to sand at $8 \mathrm{~m}$.

Threats: gill net and ropes in coral; heavily dived.

\section{Al Bustan (FM653075)}

Coral Community Type: B (site number 119)

Description: predominantly rock with scattered corals and patches Sarcophyton.

Threats: nets, ropes, litter entangled in coral; much dead/broken coral.

\section{Qantab (FM668068)}

Coral Community Type: B (site number 120)

Description: tumbled rock below cliff to $6-9 \mathrm{~m}$, covered by dense coral cover $(25-85 \%)$ including large Symphyllia colonies.

Threats: many gill nets, ropes entangled in and killing coral; $A$. planci ( 2 seen exposed, others tucked under coral); $>50 \%$ corals dead or damaged.

Bandar Jissah (General) (FM685060)

Coral Community Type: A, B (site number 121)

Description: mainly rock boulders covered by scattered corals, with small patches framework reefs (ca. $1 \mathrm{~m}$ thick) formed of Porites, Cyphastrea, P. damicornis.

Threats: numerous ropes, nets, fishing lines in coral; $A$. planci.

\section{Bandar Jissah (FM676064)}

Coral Community Type: B (site number 122)

Description: gentle rock slope from undercut rock shore to sand at $8-9 \mathrm{~m}$ covered by algal turf, alcyonarians (Sarcophyton, Sinularia) and scattered corals; towards point cover alcyonarians and corals increases to $95-99 \%$.

Bandar Jissah (Central Islet Southwest) (FM682056)

Coral Community Type: A (site number 123)

Description: narrow reef Porites with $1.5 \mathrm{~m}$ thick framework. 
Threats: knobbed smashed Porites, pieces removed.

Bandar Jissah (Central Islet North) (FM684057)

Coral Community Type: B (site number 124)

Description: steep rock slope to sand at $12 \mathrm{~m}$ with numerous small encrusting coral colonies, tabular Acropora, large Turbinaria.

Threats: very disturbed area, many corals broken or overturned; many ropes, nets in corals; $A$. planci ( 5 seen exposed).

Bandar Jissah (East Cove) (FM686053)

Coral Community Type: A, B (site number 125)

Description: banks $P$. damicornis along shore (framework 1-1.5m thick); offshore small unusual reef dominated by Cyphastrea serailia, Hydnophora exesa, Platygyra daedalea (framework ca. 1m thick); low numbers small colonies other corals.

Bandar Jissah (FM687050)

Coral Community Type: A, B (site number 126)

Description: rock platform covered by alcyonarians and scattered small coral colonies; corals larger toward point forming a narrow framework reef dominated by fused colonies of Platygyra in the north and Porites in the south.

Threats: A. planci ( 1 seen exposed).

Khawr Yenkit (FM741037)

Coral Community Type: A, B

Description: east and west shores near mouth bordered by reef of fused Porites (framework $2 \mathrm{~m}$ thick), porous due to bio-erosion, more extensive east side.

Threats: A. planci, 2 seen exposed; gill nets, ropes in reef.

Bandar Khayran (General) (FM770025)

Coral Community Type: A, B (site number 127)

Description: best coral development in Muscat area; abundant coral on rock slopes, boulders and walls; best developed framework reefs dominated by Porites, tiered Acropora reefs; almost entire shoreline of bays (excluding innermost one) fringed by rocky shores with abundant coral reaching $>75 \%$ cover in many locations; numerous patch reefs.

Threats: heavy diving activity; intense fisheries, many entangled gill nets, ropes, fishing lines, litter breaking/killing coral.

Bandar Khayran Northwest (FM 757029)

Coral Community Type: A (site number 128)

Description: reef framework of fused Porites

Threats: poor condition, much dead or damaged coral and rubble along base of reef $(10 \mathrm{~m})$; gill nets and ropes in reef. 
Bandar Khayran (Main Bay South Shore West) (FM758022)

Coral Community Type: A (site number 129)

Description: only such reef in Muscat area: dominated by Acropora spp. (bushy and tabular) forming framework $>1 \mathrm{~m}$ thick; large ( $2 \mathrm{~m}$ diameter), whorled table corals in tiers down slope bordered by bushy forms at $10 \mathrm{~m}$.

Threats: some smashed corals in shallows; many ropes in coral.

Bandar Khayran (Main Bay South Shore East) (FM760022)

Coral Community Type: A (site number 130)

Description: 2-3m thick framework of fused Porites with small colonies of other corals, leading from contiguous Acropora reef FM758022.

Threats: gill net and ropes in coral.

Bandar Khayran (Islet) (FM762023)

Coral Community Type: A, B (site number 131)

Description: rock covered $55-95 \%$ by a generally mixed assemblages of colourful corals drops to sand at 8-9m; patches may have clear predominance of e.g., Acropora, AcroporaStylophora, Porites, P. damicornis, or Pavona cactus; fused Porites reef extends west for $>100 \mathrm{~m}$ with framework thickness increasing from 0.5 to $>3 \mathrm{~m}$ below which reef slopes to sand at $12 \mathrm{~m}$; this best developed true framework coral reef in Muscat area.

Threats: ropes, nets in reef; corals broken by divers and fishermen; overturned Acropora tables.

Bandar Khayran (East Island North) (FM783026)

Coral Community Type: B (site number 132)

Description: north cove bordered by rock with algae and alcyonarians predominant; scattered corals; some large Goniopora on west side of cove.

Threats: anchors of diving boats.

Bandar Khayran (East Island Northeast) (FM784024)

Coral Community Type: A, B (site number 133)

Description: north of small beach is patch of delicately branched Montipora which leads north to a patch of dead coral followed by a small bank of $P$. damicornis; beyond this is a beautiful example of a fused Porites framework reef ( $>2 \mathrm{~m}$ thick) dropping to sand at 6 $8 \mathrm{~m}$; north of this reef are steep rock slopes and walls with scattered corals.

Threats: A. planci, 1 seen exposed; gill net in reef.

\section{Bandar Khayran (East Branch) (FM784021)}

Coral Community Type: A (site number 134)

Description: reef fringes much of rocky shore of this eastern branch of the Khayran bays complex; in centre is well formed Montipora patch reef surrounded at 4-5m depth by a fringe of Acropora (east to south), Astreopora (southwest), Hydnophora (west-centre) and Platygyra; abundant Diadema.

Threats: gill net in corals; much dead coral. 
Ra's Khayran (Sand Dunes Bay) (FM792014)

Coral Community Type: A, B (site number 135)

Description: Porites boulders fringe the south shore, fusing about midway out to form a solid flat-topped framework reef $1-2 \mathrm{~m}$ thick; reef changes considerably moving east toward mouth of bay: banks of $P$. damicornis appear along reef edge in 4-5m depth; Porites gives way to talus slope covered by small $(10-15 \mathrm{~cm})$ bushy Acropora; further out talus and boulder platform is covered by Montipora with a mixed coral assemblage along the seaward margin in $6-7 \mathrm{~m}$ depth; from here reef detaches from shore and is covered $>85 \%$ by alcyonarians (Sarcophyton predominant); this leads to mixed coral assemblage.

Threats: ropes, gill net in coral.

Ra's Khayran (East Sand Dunes Bay) (FM794014)

Coral Community Type: B (site number 136)

Description: shoaling rock shelving north to sand at $12 \mathrm{~m}$, dropping steeply seaward to $13 \mathrm{~m}$, and falling over walls, ledges, overhangs south; rock terraces covered $70-95 \%$ corals and Sarcophyton, cut by sandy gullies.

Threats: ropes, net in coral; spearfishing.

\section{Khaysat Ash Shaykh East (FM796006)}

Coral Community Type: B (site number 137)

Description: rock slope covered by fine algal turf and scattered alcyonarians and corals.

\section{Khaysat Ash Shaykh Southeast (FM798008)}

Coral Community Type: B (site number 138)

Description: cliffs fall to terrace at $7 \mathrm{~m}$ with algal turf and dense Echinostrephus molaris with few corals; area of large blocks rock tumbled together with a variety of corals; off east side rock slopes to $18-21 \mathrm{~m}$ where rocky patches covered by gorgonians.

Threats: intense gill net fishing; ropes in coral.

\section{As Sifah North (FM816972)}

Coral Community Type: B (site number 139)

Description: rocks covered by short algal turf and scattered small corals; Symphyllia common.

\section{As Sifah (FM844885)}

Coral Community Type: B (site number 140)

Description: boulder-strewn seabed with predominantly faviid corals and alcyonarians out from ca. $1 \mathrm{~m}$ below low tide mark.

Threats: intense shell and lobster collection by expatriates.

\section{Ra's Abu Da'ud (Islet) (FL960800)}

Coral Community Type: A, B (site number 141)

Description: sheer wall north side islet with small encrusting corals, and abundant Tubastrea, Dendrophyllia, Dendronephthya and gorgonians; south of islet are several large banks of dead coral (2-3m thick) with recolonisation by small $(8 \mathrm{~cm})$ colonies of $P$. 
damicornis, Acropora, Stylophora (5-25\% cover only); rock slopes toward southeast end have mixed corals reaching $50 \%$ cover.

Threats: gill nets entangled in and killing coral and large gastropods; coral breakage; spearfishing; A. planci, 2 seen exposed.

Bimmah North (GL185450)

Coral Community Type: B (site number 142)

Description: rock shelf 4-7m deep, Sarcophyton, Sinularia predominant with scattered corals, including large Turbinaria, Platygyra.

Makalla Wabar (GL282337-293304)

Coral Community Type: B (site number 143)

Description: north side beach GL282337 rock shelves and ridges angle off shore to depth 3-6m; $6 \mathrm{~m}$ shelves have alcyonarians (Sarcophyton, Sinularia) predominant with scattered small corals; shallower ridges have greater cover hard corals (Acropora, P. damicornis predominant); ca. $500 \mathrm{~m}$ offshore in $11-12 \mathrm{~m}$ depth GL287325 is low rock shelf with numerous small scattered corals, alcyonarians, and few large Astreopora and Porites; Turbinaria peltata and Pseudosiderastrea common and relatively large;

east side headland GL293304 cliff drops to terrace at $9 \mathrm{~m}$ dropping sheer to sand at $12 \mathrm{~m}$, north side rocky slope (gradient 1:1) to sand 9m; small scattered corals; Sarcophyton, Sinularia predominant.

Threats: fishing lines in corals.

Ra's North Qalhat (GL380185)

Coral Community Type: B (site number 144)

Description: rock platform at 5-6m depth cut by sheer-sided gullies with alcyonarians and scattered corals (usually $<50 \mathrm{~cm}$ colonies).

Wadi Birka Area (GL494072)

Coral Community Type: B

Description: west of wadi a series of rock shelves run parallel to the shore out to at least $10 \mathrm{~m}$ deep; shelves notably barren, supporting few scattered corals, cover $<1 \%$ nearshore ( $2 \mathrm{~m}$ depth) increasing offshore to $1 \%$ in $3-4 \mathrm{~m}, 5-7 \%$ in $7-8 \mathrm{~m}$, and $10 \%$ in $9-10 \mathrm{~m}$; proportion dead corals remains $5 \%$; opposite wadi rock shelves raised $1-2 \mathrm{~m}$ above sandy substrate, $2 \%$ cover in $4-5 \mathrm{~m}$ ( $20 \%$ dead), $5-10 \%$ in $6 \mathrm{~m}$ ( $<2 \%$ dead, tabular Acropora predominant), $70 \%$ cover in $9 \mathrm{~m}$ (3\% dead, mixed corals and alcyonarians); large Porites heads (some $>4 \mathrm{~m}$ diameter) occur just east of the wadi.

Threats: gill nets in coral; tarballs, litter, trash and sewage from Oman Sea Farms in wadi and sea; effluent from prawn ponds enhanced algal growth and apparently killed or killing $82 \%$ of Porites colonies of $>1 \mathrm{~m}$ diameter near outfall.

Bi'r Bira' West (GL520045)

Coral Community Type: A, B (site number 145)

Description: coral cover $60-70 \%$ in continuous band ca. 100m offshore GL520045544025; Montipora-Acropora dominated framework reef ( $>1 \mathrm{~m}$ thick) with spur and groove 
formations from $3-6 \mathrm{~m}$ depth; coral cover decreases gradually to $50 \%$ in $6 \mathrm{~m}$ then drops rapidly to $10 \%$ in $8 \mathrm{~m}, 5 \%$ in $9 \mathrm{~m}$ and $2 \%$ in $10 \mathrm{~m}$; shallows predominantly Sarcophyton, Sinularia; Tridacna, Lambis, Euphyllia and other corals on gravel terrace above beach, but none underwater.

Threats: gill nets in coral, overturned Acropora; land allocated for desalination plant with $1 \mathrm{~km}$ sea frontage opposite west end this well-developed and only coral reef in the area (GL520045).

Bi'r Bira' (GL533033)

Coral Community Type: A, B (site number 146)

Description: eastern extension of reef described in previous record; coral cover increases to maximum of $80 \%$ towards east before breaking apart ca. $200-300 \mathrm{~m}$ east of Bi'r Bira'. Threats: ropes, gill nets in coral; collection $L$. truncata.

Khawr South of Shiya (GK755959)

Coral Community Type: B (site number 147)

Description: scattered small coral colonies on rock.

Khawr Jaramah (GK810910)

Coral Community Type: A, B

Description: Young reefs, much dead coral.

Barr al Hikman Southeast (FH520520)

Coral Community Type: A, B (site number 148)

Description: Platygyra-Porites dominated reef with huge heads forming $100 \%$ cover in patches, and large patches whorled Montipora foliosa and Acropora; generally mixed low diversity coral assemblage; unlike reefs elsewhere in Oman; reef extends southeast of Ra's al Mishayu 100x4500m; 4May87 Sargassopsis up to 45cms long, dense Sargassopsis reef-flat southeast.

Threats: occasional gill net fishing by fishermen from Al Ashkharah; ropes in corals.

Barr al Hikman (south reefs, east side) (FH480500)

Coral Community Type: A (site number 149)

Description: east part of the large ( $>6 \mathrm{sq} . \mathrm{km}$.) reef formed exclusively of Montipora foliosa - structure this series of reefs unique in Oman and possibly the world; largest continuous coral reef in Oman, extending FH510490-420530 (>10km); framework ca. 4m thick built of huge whorled $M$. foliosa (cover 100\% over flats); where few open pools in reef find small selection of other corals; seaward margin (south-facing) protected from waves by talus banks formed of broken, dead pieces of $M$. folios $a$ and low spurs of $P$. damicornis off the reef edge in 3-5m depth; inner margin shallow (0.5-1.5m deep low neap tide), irregular contours of mounds and ridges; leads offshore to broad flat zone 70:30 live:dead coral. 
Barr al Hikman (Ra's Ghabirty West) (FH420528)

Coral Community Type: A (site number 150 )

Description: western limit of unique vast $M$. foliosa reef complex (see previous record for more details); off west edge reef fragmented patches $M$. folios $a$ and large Porites and Platygyra with small colonies other corals in $2-3 \mathrm{~m}$ over sand.

Ra's Kanasah (coral shoal south) (FH270530)

Coral Community Type: B (site number 151)

Description: shoal with coral patches ( $>50 \%$ dead) with scattered faviids predominant; edge with bank of $M$. folios $a$ west, Porites boulders north.

Ra's Shajrit (FH244594-246600)

Coral Community Type: B (site number 152)

Description: rock reef fringes shore; prolific coral growth (70\% cover) $\mathrm{FH} 245580$; Platygyra, Porites, Hydnophora, Goniopora predominant; further north rock boulders with faviids and Sinularia, live coral 30\%, alcyonarians 30\%, dead coral and rock $40 \%$.

Threats: gill net set among coral.

Jazirat Abb (FH215672)

Coral Community Type: B (site number 153)

Description: $2 \mathrm{~m}$ deep rock shelf extends out from island with patches (100-1000 sq.m.) bushy Acropora between which are rocks with massive corals (mainly faviids).

Bandar Hasik West (CE190255)

Coral Community Type: C

Description: rock boulders with algal turf, scattered corals, including: Montipora, Acropora, Pseudosiderastrea, Stylophora, faviids; Ulva.

Ra's Hasik North (CE195245)

Coral Community Type: $\mathrm{C}$

Description: coral more abundant than Bandar Hasik West; tabular Acropora 3-4m diameter, large spherical tunicates encrusting rocks.

Ra's Hasik South (CE195241)

Coral Community Type: C

Description: numerous small Stylophora, Pseudosiderastrea, encrusting Montipora in shallows, small tabular Acropora deeper.

Ra's Hasik South 1 (CE189235)

Coral Community Type: C

Description: bay bordered north and south by tumbled rock with short algal turf; Ulva; few scattered faviid corals south side; Acropora, Stylophora further out; Tubastrea $6 \mathrm{~m}$ vertical face; Dendrophyllia $3 \mathrm{~m}$ overhang; exposed north side more coral: patch small ( $<1 \mathrm{~m}$ diam) Acropora, Porites $<75 \%$ cover of rock. 
Khawr Murir North (CD018939)

Coral Community Type: C

Description: in bay rock with Sargassopsis, Ulva; outside strong surge scattered Montipora, Acropora, Stylophora.

Wadi Mahall North (BD995905)

Coral Community Type: C

Description: Acropora, Stylophora, faviids; fewer than BD990900; Sargassopsis on shallow rock outer bay; Ulva.

Threats: lines in coral; litter underwater.

Wadi Mahall (BD990900)

Coral Community Type: $\mathrm{C}$

Description: south shore Acropora, Stylophora, faviids, Turbinaria in large sheets and Favia on rock wall in inner bay; Sargassopsis outer bay and north shore inner bay; Ulva. Threats: lines in coral; much litter underwater.

Wadi Mahall South (BD986895)

Coral Community Type: C

Description: many large Porites boulders ( $>1 \mathrm{~m}$ diameter) along south shore, Acropora, Montipora, Stylophora; Sargassopsis on shallow rock; more coral here than north of Sadh; Ulva.

Threats: abundant litter.

Sadh (BD949856)

Coral Community Type: C

Description: boulders in shallows covered by stubby Sargassopsis with occasional small Stylophora; below 8m scattered Acropora and encrusting Montipora; Ulva.

Sadh South (BD941853)

Coral Community Type: C

Description: large patch Sinularia; much Eklonia on tideline; north side bay rock with algal turf, to Sargassopsis from shallows to $6-7 \mathrm{~m}$, to rock with algal turf and occasional Acropora, Stylophora to sand centre bay; south side same zonation but greater abundance and variety coral including Acropora, Stylophora, Montipora, Porites, Coscinaraea, Pseudosiderastrea, Cyphastrea, Favites sp., F. pentagona; tabular Acropora $(<1 \mathrm{~m}$ diameter); cover reaching $30 \%$ in patches; Ulva.

Sadh South 1 (BD932846)

Coral Community Type: C

Description: rock with algal turf-Sargassopsis to $6-7 \mathrm{~m}$, to algal turf and occasional Stylophora, Acropora, to deeper turf with flat calcareous rhodophytes; centre bay sandy; south side more, bigger corals than BD941853, same corals and Acanthastrea maxima; $F$. pentagona, Cyphastrea, Acropora reach >1m diameter; Ulva. 
Wadi Khorhaufa North (BD923841)

Coral Community Type: B

patchy coral (Montipora-Porites-Cyphastrea- $F$. pentagona dominant) $8-10 \mathrm{~m}$ in more exposed parts of bay.

Wadi Khorhaufa (BD914835)

Coral Community Type: C

Description: Sargassopsis, patchy Acropora, Cyphastrea, F. pentagona on south side; Ulva.

\section{Ra's Janjali Northeast (BD900828)}

Coral Community Type: $C$

Description: Inner bay very sheltered; rock with Padina, numerous Echinometra; outer bay Sargassopsis, Favites sp., F. pentagona, Acropora; Ulva; below this zone larger colonies coral including Montipora, Cyphastrea, 4+m diameter Leptastrea with 11 slipper lobsters underneath.

Bandar Qinqari East (BD891827-890812)

Coral Community Type: C (site number 155 )

Description: below 6m Acropora, Montipora, faviids in exposed location 15-20\% cover; at $10 \mathrm{~m} \mathrm{30-40 \%} \mathrm{cover} \mathrm{with} \mathrm{blunt-branch} \mathrm{Porites,} \mathrm{Turbinaria,} \mathrm{Platygyra,} \mathrm{Cyphastrea,}$ Leptastrea (>2m diameter), H. exesa; Sargassopsis shallower; Ulva.

Wadi Bayt Said East (BD828795)

Coral Community Type: A, C (site number 156)

Description: Tiers tabular Acropora (2-3m diameter) east side from $3 \mathrm{~m}$ to $6-7 \mathrm{~m}$ deep, $75 \%$ cover, large Porites ( $2 \mathrm{~m}$ diameter, with 1 of $4 \mathrm{~m}$ ); further out bay rock terrace $9 \mathrm{~m}$ deep with dense coral cover down to sand $13 \mathrm{~m}$; similar west side but coral band narrower. Threats: lines in coral.

Wadi Bayt Said West (BD782790)

Coral Community Type: C

Description: generally algal turf with Padina inner bay; sparse Sargassopsis, scattered Stylophora outer bay with small tabular Acropora deeper; large area coral 20-30\% cover in cove at mouth east end BD793784; large heads Porites, Cyphastrea, tabular Acropora scattered along rocky borders of cove BD790789.

Wadi Aingalf East 3 (BD756770)

Coral Community Type: A, B, C (site number 157)

Description: cover $40-60 \%$ from 6 to $15 \mathrm{~m}$.

Threats: lines in coral.

Wadi Aingalf East 2 (BD752769)

Coral Community Type: A, B, C (site number 158)

Description: gradual slope (gradient 1:5) with coral from 5-6m to bay floor $10 \mathrm{~m}$; at $6-7 \mathrm{~m}$ 
Acropora-Montipora assemblage cover $80-90 \%, 9-10 \mathrm{~m}$ coral cover $50-80 \%$; definite reef framework; inner bay Acropora-faviid assemblage at $5 \mathrm{~m}$.

Threats: lines in coral.

Wadi Aingalf East 1 (BD748767)

Coral Community Type: A, B, C (site number 159)

Description: See BD746773 for inner bay; corals begin 8m, terrace at $9 \mathrm{~m}$ with gentle slope to $15 \mathrm{~m}$ with $60 \%$ cover coral in patches, extend down sides, ends rock ridges to $17 \mathrm{~m}$; some patches dominated by large $H$. exesa or Pavona or Echinophyllia.

Threats: gill net, lines in coral.

Wadi Aingalf East (BD746773)

Coral Community Type: C (site number 160)

Description: See BD748767 for details outer bay; rock with algal turf to $8 \mathrm{~m}$; east side $8 \mathrm{~m}$ down Acropora, Cyphastrea, F . pentagona, cover 5-50\%; tabular Acropora predominant; west side less coral; Ulva.

Wadi Ayn East (BD727766)

Coral Community Type: A, B, C

Description: west side few scattered corals outer bay, huge Porites ( $3-4 \mathrm{~m}$ diameter, one $4-5 \mathrm{~m}$ ) in $2-4 \mathrm{~m}$ depth inner bay; centre bay patch reef formed on sand of large Porites fused together, other corals where Porites dead; east side $<20 \%$ cover coral encrusting rock slope, patchy.

Threats: lines, gill net in coral.

Wadi Ayn (BD724768-726765)

Coral Community Type: B, C

Description: rock with silty algal turf-Stylophora-Acropora-Porites from $2 \mathrm{~m}$ to sand $7 \mathrm{~m}$ where much Padina; further out gullied rock with sides, rocky bottoms gullies coral encrusted (predominantly table Acropora); Ulva.

Wadi Ayn West (BD709768)

Coral Community Type: A, B, C

Description: small patch reef; rock with algal turf-Sargassopsis-?Dictyota, Ulva, occasional Stylophora, Acropora, faviids increasing in abundance in centre bay; toward west-centre bay coral cover $40-60 \%$ at $8 \mathrm{~m}$; in mouth bay, rocks bay floor corals fewer; large patches Sinularia.

Raaha (Hoon's Bay) East (BD664749)

Coral Community Type: A, B, C (site number 161)

Description: Bay sheltered by shallow rock spur across mouth; true reef framework, extensive coral growth extending to outer bay on $9 \mathrm{~m}$ terrace; inner bay shallow algal covered coral; much Sargassopsis outer, Ulva (see next record for west side bay).

Threats: lines, ropes in coral; coral heads used to weight fish traps; spearfishing, broken coral, tarballs, litter underwater; litter ashore, oil on rocks. 
Raaha (Hoon's Bay) West (BD663747)

Coral Community Type: A, B, C (site number 162)

Description: true reef framework with cover up to $99 \%$; $3 \mathrm{~m}$ terrace dominated by Acropora; reef developed over terrace, down rock slope to $10 \mathrm{~m}$; large Porites, Pavona heads (2-5m diameter); further out rock spur with dropoff to $12 \mathrm{~m}$, Stylaster abundant.

Threats: lines in coral; broken coral, selective spearing groupers.

Raaha West 1 (BD660747)

Coral Community Type: A, B, C

Description: Inner bay: patchy scattered coral on rock 5-40\% cover, primarily Acropora, large Platygyra, branching Porites; outer bay east side: small patch branched and massive Porites forming veneer framework, 95\% cover live coral on rock slope and smaller less consolidated patches Pavona; outer bay west side: stepped rock shelves, ledges, cover 0-60\%, primarily Platygyra and branched Porites, decreasing from outer to inner bay.

Raaha West 2 (Eagle's Retreat) (BD654743)

Coral Community Type: B, C (site number 163)

Description: algal turf-Sargassopsis and scattered Acropora-F. pentagona on rock outcrops closer to shore and few Montipora, encrusting Porites; crevices packed Echinometra, Stomopneustes, large tunicates, large octopi; further offshore outcrops from sand at $5-10 \mathrm{~m}$ depth to $3 \mathrm{~m}$ deep running parallel shore from rock islet at north end; coral encrusted spurs, gullies south small beach; further out tumbled rock slope with large Turbinaria, patchy tiers tabular Acropora 5-9m; shown fungiid allegedly found here.

Threats: lines in coral; coral broken by divers.

Raaha West 3 (BD650749)

Coral Community Type: B, C

Description: tumbled rock with scattered Acropora (predominant), Turbinaria, smaller colonies faviids; cover $<5 \%$ increasing in inner bay to $20-30 \%$ with predominantly massive corals (Porites, Goniopora, F. pentagona), Stylophora in 1.5-3m; east side rock scoured bare.

Threats: lines in coral.

\section{Wadi Anshayr East (BD611766)}

Coral Community Type: A, B, C

Description: east side large Porites patch reef $>2 \mathrm{~m}$ thick, $40 \%$ live coral cover - rest dead coral (not rock!); Porites heads (1-1.5m diameter) scattered through cove and in shallows; rocks with scattered Acropora, faviids, Coscinaraea; shallows Porites forms reef framework up to $1 \mathrm{~m}$ thick; Ulva.

Khawr Hino West (BD601760)

Coral Community Type: B, C

Description: rock slope 1:3 gradient, corals patchy, scattered; cover to $70 \%$ in 1 patch; Acropora predominant on sides, bottoms gullies; inner bay sand-rock pavement; few small corals. 
Hino South (BD596760)

Coral Community Type: C

Description: shallow rock with silty algal turf, turbid water with decaying algae; at $2 \mathrm{~m}$ algae with few scattered small corals (Stylophora, $F$. pentagona); at 3-4m scattered Acropora, Favia, Favites, Cyphastrea, Goniopora; Sargassopsis, Ulva; 50cms deep Sargassopsis on tidelines 25Aug88.

Jazirat Hino West (BD594755)

Coral Community Type: B, C (site number 164)

Description: shallow rock around island with Sargassopsis, scattered corals to 4-5m; below this more coral; coral more abundant west side: rock slope edge channel 40-60\% cover, reaches $90 \%$ centre west bay, huge Porites (12m diameter $3 \mathrm{~m}$ thick); 24 Aug88 mainland shore piled 50cms thick Sargassopsis; Ulva.

Threats: coral heads used to weight fish traps.

Marbat South (BD545780)

Coral Community Type: C

Description: rocky outcrops with scattered coral, predominantly tabular Acropora, large Turbinaria, faviids, $2 \%$ cover; large sand patches.

Ra's Marbat (BD530786)

Coral Community Type: B (site number 165)

gullied rock with algal turf, scattered coral, $2 \%$ cover, including unusual deeper water varieties.

Marbat (BD536793) A, C

Description: small incipient patch reef formed fused Porites colonies, 2-3m diameter (S-centre cove); centre cove rock outcrops with scattered Acropora, Montipora, faviids, occasional large Platygyra (1-2m diameter), Porites; Porites generally $<1 \mathrm{~m}$ diameter but larger, more abundant under lee south rock point (1-2m diameter); bay floor rock with algal turf, small sand patches, scattered coral; fewer corals north side cove.

Threats: lines, gill net in coral.

Ra's Raysut (Raysut Rock) (AD815749/ZU208749)

Coral Community Type: C (site number 166)

Description: east side: rock shelf $9-12 \mathrm{~m}$ gradual slope, sheer-stepped to $>16 \mathrm{~m}$ with overhangs, shallow caves; south side: more scenic with sandy gullies, caves to sand at 15$16 \mathrm{~m}$; rock with short algal turf, scattered encrusting corals, tunicates; deeper caves/overhangs south side with black coral (Antipathes sp.) - killed by entangling lines/ropes in places; few coral types.

Threats: ropes, lines tangling marine life, killing antipatharians; old ropes, fishing lines, litter underwater. 
Raysut West (Rock Stack) (ZU190740)

Coral Community Type: B (site number 167)

Description: rock blocks, gullies with short algal turf, scattered corals $<5 \%$ cover.

Threats: lines in coral.

Raysut West 1 (ZU173730)

Coral Community Type: B

Description: rock outcrops with short algal turf, scattered encrusting corals, generally $5-15 \%$ cover, $25 \%$ maximum; Ulva.

Ra's Hamar (ZU140710)

Coral Community Type: B (site number 168)

Description: steep rock terraces $9-12 \mathrm{~m}$ deep with short algal turf, scattered coral; gravel-shell talus down slope with corals, other encrusting organisms; cave at $15 \mathrm{~m}$ below which sand-rock slopes steadily deeper.

Threats: lines in coral, rock.

Fizayih (YU892631-888625)

Coral Community Type: B (site number 169)

Description: sides rock outcrops encrusted by coral, scattered corals top; north side point sheltered, luxuriant coral patches, $50-90 \%$ cover (Platygyra $<2 \mathrm{~m}$ diameter).

Ghubbat al Fizayih West (YU846574-850584)

Coral Community Type: B (site number 170)

Description: YU852576 large rock blocks, caves, narrow sheer-sided gullies, encrusted corals, abundant large fishes (including large spotted Dermatolepis), Echinostrephus, seafans, some black coral; sides gullies/overhangs encrusted Tubastrea, Stylaster.

Threats: lines in coral.

Ra's Sajir East (YU795555)

Coral Community Type: B

Description: flat rock encrusted 25\% with Montipora increasing to $50 \%$ on rock boulders, outcrops where Montipora, tabular Acropora, faviids, Pavona (massive, untidy), Leptastrea, H. microconos; Echinostrephus exceedingly abundant.

Wadi Nhaart East (Millepora Bay) (YU710544)

Coral Community Type: B (site number 171)

Description: large boulders nearshore to rock shelves, outcrops with abundant coral; coral cover 25-50\% maximum; corals encrust rock with Platygyra as low colonies $>1 \mathrm{~m}$ diameter, corals seen include table Acropora, Platygyra, Montipora, $H$. exesa, massive blade Pavona, Porites, Millepora; Millepora and scattered corals down to 5-6m; at 6-9m variety corals to $25 \%$ cover; $9-15 \mathrm{~m}$ Echinopora predominant, $<65 \%$ cover. 
Ma'alqawt (YU700546-693548)

Coral Community Type: B (site number 172)

Description: rock ridges, gullies, outcrops with algal turf, scattered corals.

Kharfot (YU488508)

Coral Community Type: $\mathrm{C}$ (site number 173)

Description: Offshore at $12 \mathrm{~m}$ deep $90 \%$ cover Sargassum with small rock shelves with sponges and very small scattered corals.

\section{Shinays (DE087477)}

Coral Community Type: B

Description: Bare rock and rock with strips Sargassopsis; scattered small colonies Stylophora, Porites, Acropora, F. pentagona and other faviids, Montipora.

\section{Al Hallaniyah (Northwest Bay) (CE900364)}

Coral Community Type: B, C (site number 175)

Description: Bay bordered by rock, encrusting coral; extensive coral gardens south side; occasional huge Porites; sandy seabed with rock ridges, dominates, coral-encrusted or with Sargassopsis, coral cover 0-75\% live.

Threats: gill net in coral.

\section{Al Hallaniyah Southeast (DE032355)}

Coral Community Type: A, B, C (site number 176)

Description: Extensive coral in bay, banks Stylophora in sheltered coves, Porites heads in solid fused banks; tiers Acropora; rocky slopes covered by variety smaller coral colonies; some areas coral cover 99\% live; breeding area Chaetodon vagabundus and Megaprotodon strigangulus.

Threats: net in and breaking coral.

\section{Al Hallaniyah South 2 (CE929338)}

Coral Community Type: B, C (site number 177)

Description: Inner bay: boulders with luxuriant Sargassum, scattered bushy and tabular Acropora, Porites; further out vast tabular Acropora, Porites (some fused) and Platygyra; outer bay: depth 7-8m, more varied corals, occasional large Porites; <99\% cover; vertical walls Tubastrea, Echinophyllia, Blastomussa.

\section{Al Hallaniyah (Southwest Bay) (CE911342)}

Coral Community Type: A, B, C (site numbers 178, 179)

Description: Inshore large boulders with luxuriant algae, small colonies corals Porites, Montipora, $F$. pentagona; large shoals scarids, carangids, siganids, Diplodus; $6 \mathrm{~m}$ shelf 90\% coral cover (50:50 hard:soft); coral reef dominated by extensive spur fused Porites $1-1.5 \mathrm{~m}$ thick, $100 \%$ coral cover, $70-80 \%$ live.

Threats: net in and breaking coral. 
As Sawda (CE780350)

Coral Community Type: A, B, C (site number 180)

Description: reef framework west bay.

As Sawda Northeast (CE808346)

Coral Community Type: B, C (site number 181)

Description: Tabular Acropora forms large patches $70-90 \%$ live coral in tiers; patches Porites ( $>2-3 \mathrm{~m}$ diameter) with bushy Acropora, Platygyra, large Goniopora, Turbinaria; coral extends out of bay to sides/bases rock spurs, where smaller, more varied colonies.

As Sawda East (CE808334)

Coral Community Type: A, B, C (site number 182)

Description: Scattered Porites in bay, and bushy Acropora; Porites heads further out and encrusting coral; extensive tiers tabular Acropora, large dead area; outer bay much live coral, dropoff to coral-covered floor at $12 \mathrm{~m}$.

As Sawda South (CE781336)

Coral Community Type: A, B, C (site number 183)

Description: Coral scattered over shallower rocks; framework $(<1 \mathrm{~m}$ thick) fused Porites in sheltered west coves; bay floor $6-13 \mathrm{~m}<80 \%$ coral cover over vast area; deeper corals dominated by large Echinopora (about 50\%), Montastrea curta; Millepora along rock fringe; extensive tiers tabular Acropora.

As Sawda (West Bay) (CE758344)

Coral Community Type: A, B, C

Description: bay lined by Porites (boulders, knobbed, branching), forms 1-2m thick framework platform along south shore, dead on top; banks largely dead coral centrally with small Stylophora (30-95\% dead); north side much dead branching coral, Porites in outer reaches, inner part Porites-Turbinaria (alga), numerous Goniopora colonies; large 2-4m diameter Acropora tables, Porites boulders at mouth bay.

\section{As Sawda Northwest (CE760350)}

Coral Community Type: B, C (site number 184)

Description: west side inner bay Stylophora forms wide flat "micro-atolls" and few small Porites; bay floor sand, small rock boulders; several dead very large table Acropora, much live table Acropora west side near headland; rock boulders off west headland covered (50-80\%) corals, mainly table Acropora (2-3m diameter), Platygyra. 
APPENDIX 3. SUMMARY OF CORAL BLEACHING OBSERVATIONS, MUSANDAM (grid coordinates from National Survey Authority Map Series K6611, $1: 100,000)$

Jazirat Habalayn (DP357938):

Air Temperature:

$22 / 07 / 90 \quad 39^{\circ} \mathrm{C}(1400 \mathrm{hrs})$.

\section{Sea Temperature:}

$22 / 07 / 9034.5^{\circ} \mathrm{C}\left(1400 \mathrm{hrs}\right.$ ) at surface to $1 \mathrm{~m}$ depth, gradually cooling to $32^{\circ} \mathrm{C}$ at $10-12 \mathrm{~m}$.

$25 / 08 / 9033^{\circ} \mathrm{C}(1430 \mathrm{hrs})$ at surface; $32.5^{\circ} \mathrm{C}$ at $5 \mathrm{~m} ; 32^{\circ} \mathrm{C}$ at $8 \mathrm{~m}$.

$20 / 10 / 9030^{\circ} \mathrm{C}(1530 \mathrm{hrs})$ at surface.

Observations:

22/07/90 Colonial zoanthids bleached white; minor bleaching of some Acropora, Montipora, and $P$. cactus to noticeably pale, but not white.

25/08/90 All Stylophora bleached to pale yellow, and all Acropora and zoanthid Palythoa to bright white; some Porites, P. cactus, Echinopora, and anemones bleached white, and Astreopora pale blue. Exposed horizontal surfaces of coral colonies more bleached than vertical or shaded ones.

20/10/90 Corals largely recovered, but picture confused by Acanthaster predation. Palythoa dead, discoloured and sloughing off rock; Symphyllia and anemones remain bleached but alive and responsive to stimulus; $<20 \%$ Stylophora and Acropora in shallows dead and covered by fine filamentous alga; remaining Stylophora and Porites have recovered normal colouration, showing no sign of bleaching; 80\% Echinopora still bleached, $30 \%$ dead; $50 \%$ P. cactus dead.

Jazirat Maqlab (DP344974):

Air Temperature:

$26 / 07 / 90 \quad 38^{\circ} \mathrm{C}(1200 \mathrm{hrs})$.

$22 / 08 / 9039^{\circ} \mathrm{C}$.

$21 / 10 / 90 \quad 34^{\circ} \mathrm{C}$.

\section{Sea Temperature:}

$26 / 07 / 9033^{\circ} \mathrm{C}$ (1200 hrs) surface to $1 \mathrm{~m}$; gradually cooling to $31^{\circ} \mathrm{C}$ at $10-12 \mathrm{~m}$.

$22 / 08 / 90 \quad 33.5^{\circ} \mathrm{C}$ at surface; $32.5^{\circ} \mathrm{C}$ at $12 \mathrm{~m}$.

$21 / 10 / 90 \quad 30^{\circ} \mathrm{C}$ surface to $10 \mathrm{~m}$. 
Observations:

26/07/90 No sign of coral bleaching.

22/08/90 Fine-branched Acropora severely bleached all around island; patchy bleaching of stout-branched Acropora, and table Acropora; Montipora and $P$. damicornis bleached white; some $P$. cactus bleached to pale yellow-brown.

21/10/90 Extensive bleaching-related mortality to Acropora; fine-branched Acropora $60-90 \%$ dead from patch to patch; stout-branched Acropora $<2$ $\mathrm{m}$ depth $60 \%$ dead, $>2 \mathrm{~m}$ depth largely unaffected, but some patches $75 \%$ dead down to depth of $5 \mathrm{~m}$; table Acropora about $25 \%$ dead; $P$. damicornis $12 \%$ dead (only one large colony present). P. cactus, Porites, Platygyra, and all other corals have recovered normal colouration.

Jazirat Sibi (DQ391001):

Air Temperature:

$22 / 08 / 9039^{\circ} \mathrm{C}$.

$21 / 10 / 90 \quad 34^{\circ} \mathrm{C}$.

\section{Sea Temperature:}

$22 / 08 / 90 \quad 34^{\circ} \mathrm{C}$ surface to $1 \mathrm{~m}$; gradually cooling to $33^{\circ} \mathrm{C}$ at $10 \mathrm{~m}$.

$21 / 10 / 90 \quad 31^{\circ} \mathrm{C}$ surface to $3 \mathrm{~m}$.

Observations:

22/08/90 Extensive bleaching of fine-branched Acropora down to $3 \mathrm{~m}$ depth; stout-branched Acropora patchy bleaching; table Acropora little affected; Stylophora bleached; no bleaching below $5 \mathrm{~m}$ depth. Bleaching variable from pure white to pale yellow, with upper parts of branches paler than shaded lower sides. Some branched dead and covered by sparse cover of fine filamentous alga.

21/10/90 Only Acropora shows mortality. Acropora mortality down to $3 \mathrm{~m}$ depth averages about $80 \%$, with patches of fine-branched, stout-branched and tabular species all affected. Mortality varies between $60 \%$ and $98 \%$ from patch to patch. Pavona, Porites, and faviids all have normal colouration.

Khawr Quway' (DQ365160):

Sea Temperature:

$22 / 08 / 90 \quad 32^{\circ} \mathrm{C}(1400)$ surface to $6 \mathrm{~m}$.

$21 / 10 / 9029^{\circ} \mathrm{C}(1300)$ surface to $1 \mathrm{~m}$. 
Observations:

22/08/90 NE side shelf below small beach: Porites over reef-flat bleached to yellow or pale blue, extensive but not severe; some zoanthid Palythoa colonies bleached to pale yellow-brown with dead patches turned green by epiphytic alga.

24/08/90 W side along Jazirat Umm al Ghanam: luxuriant Acropora and Montipora reef with extensive bleaching of Montipora, but Acropora unaffected. Colour of bleached Montipora grades from pale brown to near white or very pale blue.

21/10/90 Some mortality of table Acropora $(<1 \%)$ that may be bleach related. All other corals show normal colouration, including the formerly badly affected Montipora.

Maqaqah (DP376977):

Sea Temperature:

$25 / 08 / 90 \quad 35^{\circ} \mathrm{C}$ surface; $34^{\circ} \mathrm{C}$ at $3 \mathrm{~m}$.

$20 / 10 / 9031^{\circ} \mathrm{C}$ surface to $3 \mathrm{~m}$.

Observations:

25/08/90 Branching Acropora, P. cactus, Leptastrea, Favia, Favites and Platygyra all bleached to pale yellow or white. Some Platygyra dead and covered by sparse fine filamentous alga and sediment deposit. Porites unaffected.

20/10/90 Favia and Favites recovered from bleaching. P. cactus about $10 \%$ dead and some still bleached but apparently recovering; Platygyra $30-40 \%$ dead, mostly on dorsal surface which now covered by alga and silt.

Nibah (DP430854):

Sea Temperature:

$20 / 10 / 90 \quad 29^{\circ} \mathrm{C}$ at surface.

Observations:

20/10/90 Minimal bleaching of Echinopora and Favites. No other corals affected, no mortality.

Ra's Marovi (DP432742):

Sea Temperature:

$20 / 10 / 90 \quad 29^{\circ} \mathrm{C}$ at surface. 
Observations:

20/10/90 No evidence of bleaching.

Zaghi South (DP276417):

Sea Temperature:

$20 / 10 / 9031^{\circ} \mathrm{C}(0900 \mathrm{hrs})$ surface to $5 \mathrm{~m}$.

Observations:

20/10/90 Table Acropora $90-95 \%$ dead above $3 \mathrm{~m}$ depth; below $3 \mathrm{~m} \mathrm{95 \%}$ alive; Platygyra 1-2\% bleached and dead, many alive but dorsal surface bleached to pale brown; Symphyllia bleached to pale yellow but alive.

$R a$ 's Sawt (DP279410):

Sea Temperature:

$20 / 10 / 9031^{\circ} \mathrm{C}(0930 \mathrm{hrs})$ surface to $5 \mathrm{~m}$.

Observations:

20/10/90 All Sinularia, and some Symphyllia and $P$. damicornis bleached but alive; about $1 \%$ of Platygyra bleached and dead. In the outer bay all corals healthy. In the inner bay all table Acropora shallower than $3 \mathrm{~m}$ was dead, patches at $3 \mathrm{~m} 20-50 \%$ bleached. 


\section{$2 \mathrm{BHL}$ Biodiversity Heritage Library}

Salm, Rodney V. 1993. "Coral reefs of the Sultanate of Oman." Atoll research bulletin 380, 1-85.

View This Item Online: https://www.biodiversitylibrary.org/item/123414

Permalink: https://www.biodiversitylibrary.org/partpdf/83146

\section{Holding Institution}

Smithsonian Libraries

\section{Sponsored by}

Biodiversity Heritage Library

\section{Copyright \& Reuse}

Copyright Status: In Copyright. Digitized with the permission of the rights holder Rights Holder: National Museum of Natural History, Smithsonian Institution License: https://creativecommons.org/licenses/by-nc-sa/4.0/ Rights: http://www.biodiversitylibrary.org/permissions/

This document was created from content at the Biodiversity Heritage Library, the world's largest open access digital library for biodiversity literature and archives. Visit BHL at https://www.biodiversitylibrary.org. 